\title{
Abstracts of the XXVII Medical Academic Congress of Unicamp, COMAU, 2018
}

\section{Editorial}

Isabella Batistela Inhesta Sacho, Andrelisa Marina de Albuquerque.

During October 26th and 27th, the Student Council Adolfo Lutz academic institution consisting of medicine undergraduates - held the XXVII Medical Academic Congress of Unicamp (in Portuguese, CoMAU). It took place in the School of Medical Sciences (in Portuguese, FCM) of the University of Campinas (Unicamp), considered by national and international rankings, the best university in Latin America for two consecutive years.

According to the current dean of FCM, Prof. Dr. Luiz Carlos Zeferino, "[...] we occupy the first position in rankings that evaluate education quality. This is very relevant [...] when you look at FCM route, you start seeing its potential from wider points of view, such as from social policies and from academic and professional schooling. Our potential is wide".

The organization of COMAU is annually tutored by Prof. Dr. Egberto Ribeiro Turato, professor of Medical Psychology and Psychiatry Department of FCM, and it is the biggest event held by medicine students, directed at undergraduates, graduates, residents and other professionals of health sciences. The Congress consisted of lectures about obesity, feared diseases nowadays, children health, chronic pain and death, and also workshops on clinical abilities, ultrasound, basic life support, airway handling in emergency, trauma and obstetric emergency.

The event was opened by the lecture "Drug use today and its consequences for human being and society" given by the illustrious historian Prof. Dr. Leandro Karnal to a public of more than 300 people. "I thank the students, mainly, that make possible the survival of plural debate, controversy and democratic, which is origin and shelter of every truth idea. While it still exists, we will be able to have essential insights, so let us seize this moment, now" declared the lecturer.

"I always had the dream of studying at this university. This moment is very important to me. Unicamp is the greatest university in Latin America and our challenge has been to organize a congress as great as this university", said the $3^{\text {rd }}$ year medicine undergraduate, Arthur José

Roque Cruz. He is the vice president of the Student Council Adolfo Lutz and president of XXVII CoMAU's Organizer Commission.

"The undergraduate course in medicine reached 330 applicants per place at the college entrance exam. Since the beginning, we have excellent students", added Marcelo Knobel, rector of the University of Campinas.

In 2018, CoMAU had a record number of participants presenting scientific projects. There were 129 projects enrolled, $57,32 \%$ more than in 2017.

"The current edition focused on a high-quality scientific program, which resulted in great success of public and elevated number of scientific projects" concluded Zeferino, congratulating the Congress organizers, especially Diego Modesto Alves and Isabella Batistela Inhesta Sacho, directors of Research in the Student Council and also of the Scientific Committee of CoMAU 2018.

Pictures of the event can be seen in:

https://www.facebook.com/comau.unicamp/?ref=bookmarks.

\section{ADOLFO LUTZ PRIZE}

In the evening of October 27th, the winners of the Prize were announced:

1- Category Basic Science:

2- Category Internal Medicine:

3- Category Pediatrics:

4- Category Gynecology and Obstetrics:

5- Category Social Medicine:

6- Category Surgery:
Caio Augusto Leme Fujiura Augusto César Bortoluci Natália Rocha Tardelli Rafaella Colicigno Rebelo Seok Woo Shin Fernanda Dias Teramoto 



\section{Abstracts of the XXVII Medical Academic Congress of Unicamp, COMAU, 2018}

01.

\author{
BILATERAL INGUINAL HERNIORRHAPHY (BIH) LAPAROSCOPIC \\ TRANSABDOMINAL PREPERITONEAL PATCH PLASTY (TAPP) WITH THREE- \\ DIMENSIONAL MESH AND FIXATION WITH CYANOACRYLIC GLUE. \\ Isabela Souza Guilherme, José Homero Soares, João Pádua \\ Manzano, Carolina de Araújo Oliveira
}

The definitive treatment of inguinal hernia is surgical, however, it has been a controversial area in the surgical practice. In view of the lack of intervention, the aim of this study was to present a sample of 10 operated cases, all male, bilateral hernia with the same technique: laparoscopic transabdominal preperitoneal patch plasty (TAPP) inguinal herniorrhaphy with three-dimensional polypropylene $3 \mathrm{D}$ monofilament mesh fixed with cyanoacrylic-based synthetic surgical glue. A favorable evolution was observed, receiving a 24-hour hospital discharge after the technique procedure. Any postoperative complications were observed, discomfort or pain were minimal or absent. The technique proved to be effective in the repair of inguinal hernias being minimally invasive allowing the patient to return to their activities a few days after surgery. Although the monitoring period consists of only three years, the study has no recurrence rate until the present moment.

02.

\section{TELEMEDICINE IN THE SCREENING OF PATIENTS WITH SUSPECTED SPINE TRAUMA}

Bruno Santos Bogea, Andrei Fernandes Joaquim, Alexsandro Nascimento Sobrinho, Luiz Adriano Esteves, Marcelo Comes Cordeiro Valadares.

One of the great challenges of medicine is the guarantee of fast and efficient access to treatment with optimization of the resources. The grea technological development, mainly referring to the transmission of data and images, has proved to be an important tool in the exchange of medical information. In many countries, telemedicine is used to organize patient flow and guide distance treatment in services where there is no readily available specialist physician. Considering the importance of telemedicine in optimizing the transfer and screening of patients, the present study aims to evaluate the safety of previous screening by imaging of patients with suspected spinal cord injury in services with no specialist available. Five independent neurosurgeons have retrospectively evaluated clinical and radiological data from patients with suspected MRS treated in small hospitals who were referred to the Hospital das Clínicas of UNICAMP in a telemedicine setting. They were diagnosed and guided the distancescreening procedure for these patients. The performance of these professional evaluators by telemedicine was compared to the evaluation of the neurosurgeon professor doctor specializing in spinal cord trauma who attended the patients in loco.

03.

CENTRAL PONTINE MYELINOLYSIS AFTER LIVER TRANSPLANTATION: CLINICAL CONDITION, ASSOCIATED FACTORS AND TREATMENT. RETROSPECTIVE ANALYSIS OF 20 YEARS.

Fernanda Dias Teramoto, Elaine Cristina de Ataide, Simone Reges Perales

INTRODUCTION: Central Pontine Myelinolysis (CPM) is a rare neurological disease characterized by symmetric and non-inflammatory demyelination of the central region of the bridge. Patients undergoing liver transplantation are more susceptible to the development of CPM due to frequent electrolytic imbalances during surgery, elevated serum immunosuppressive concentration and previous hepatic encephalopathy. It is known that such patients present higher mortality rates and worse prognoses. OBJECTIVES: In this context, the objective of this study is to present a description of the population with a diagnosis of CPM after liver transplantation in Clinical Hospital of Unicamp from 1997 to 2017, based on the analysis of several epidemiological and clinical variables associated with the development of the disease and the literature review of the theme. PATIENTS AND METHODS: The medical records of patients submitted to liver transplantation with a diagnosis of CPM were evaluated in the Clinical Hospital of the State University of Campinas and data regarding the population and clinical profile of these patients were analyzed. RESULTS: Eight CPM cases after hepatic transplantation were identified, which correspond to an incidence of $0.94 \%$. The mean age of the patients was 50.25 years $( \pm 13.6)$ and the majority $(62.5 \%)$ of the patients were male. In the sample, $62.5 \%$ of the patients had a history of alcoholism, and $37.5 \%$ presented alcoholic cirrhosis as the main etiology of liver disease. Hepatic encephalopathy and ascites were the most frequent associated clinical conditions $(87.5 \%)$. The pre and postoperative values of serum sodium were $135.63( \pm 3.16)$ and $145.25( \pm$ 8.19 ), and 3 patients presented hyponatremia preoperatively and 5 patients presented hypernatremia in the post- operative. The mean onset time of symptoms from the transplant was 6.5 days $( \pm 4.63)$, which compatible with the time reported in the literature. The symptoms presented were diverse, from changes in level of consciousness and motor manifestations to psychiatric manifestations. The diagnosis of all patients was essentially clinical, and only $62.5 \%$ of the patients presented clinical conditions and the possibility of access to magnetic resonance imaging (MRI). Among them, all presented typical lesions. Considering that many patients with CPM are asymptomatic or have mild symptoms, it is possible that the number of diagnoses is underestimated in this population. The treatment was based on steroid therapy in $87.5 \%$ of the cases. As a prognosis, $50 \%$ of the patients evolved to death, and the mean survival was 83.3 days $( \pm 85.56)$. Only 1 patient progressed with complete improvement without sequelae. CONCLUSION: It was concluded that the population profile of these patients presents similarities with the profiles described in the current literature. Due to the wide variety of possible clinical manifestations in the disease and therefore to the diagnostic difficulty, greater access to the confirmatory examination (MRI) could reduce the number of underdiagnoses.

04. PREVALENCE OF OCCULT METASTASES AND CERVICAL LYMPHATIC DRAINAGE PATTERN DETECTED BY SENTINEL LYMPH NODE MAPPING IN PAPILLARY THYROID CARCINOMA.

Samuel Serpa Steck, Jose Higino Steck

INTRODUCTION: Papillary thyroid carcinoma (PTC) is the most common endocrine neoplasia of the human being, presenting an overall increase in its incidence in the last decades. The prevalence of cervical lymphatic metastases in PTC ranges from $30 \%$ to $90 \%$ in different studies. The large variation found in the literature about the prevalence of cervical lymph node metastases in the PTC at the initial diagnosis also justifies the search for a cervical lymphatic drainage pattern of this type of tumor and that may assist the surgeon in the search for occult metastases. OBJECTIVES: This study aims to verify: 1) the prevalence of occult cervical metastases detected by sentinel lymph node mapping (SLNM) in the PTC and 2) the cervical lymphatic drainage pattern of the PTC. METHODS: This is a cross-sectional study of 38 cases of PTC attended in a single center. All patients were submitted to total thyroidectomy, SLNM with radiopharmaceutical and elective central compartment cervical drainage. Results of SLNM, together with the anatomopathological examination, were evaluated to determine the prevalence of occult cervical lymphatic metastases and the cervical lymphatic drainage pattern of papillary thyroid carcinoma. RESULTS: SLN was mapped in $95 \%$ of the cases and in $48.5 \%$ occult metastases were found. The lymphatic cervical drainage pattern is associated with the location of the primary tumor. In 2 thirds of the series, SLN was found in central compartment (CC), whereas in 1 third of the cases SLN was found in CC and in lateral compartment. CONCLUSIONS: 1) The prevalence of occult cervical metastasis diagnosed by SLNM was $48.5 \%$. 2) The drainage pattern varied according to the location of the primary tumor; tumors of the middle third and isthmus had greater drainage for CC; tumors of the upper and lower third drained to $\mathrm{CC}$, but also to $\mathrm{CC}+$ lateral compartment.

05. HEPATOCELLULAR ADENOMA: PHENOTYPIC EXPRESSION, CASUISTRY DESCRIPTION AND LITERATURE REVISION Gabriella Junis Noyma, Simone Reges Perales 
INTRODUCTION: Hepatic Adenoma (AH) is a rare and benign tumor, usually solitary, more common in females and more prevalent in reproductive age, characterized by a benign proliferation of hepatocytes with a discrete fibrous capsule, besides not containing portal elements. The presence of nuclear atypia is infrequent and this finding should raise the suspicion of tumor malignancy. Clinically it shows with little expressive symptomatology, it is usually reported abdominal pain in the epigastrium and / or right hypochondrium, and it may evolve to rupture, one of the feared complications. The objective of this study is the search for predictive elements of poor prognosis in $\mathrm{AH}$ for better characterization of the pathology, as well as to improve the sensitivity of indications for surgical treatment of these tumors. RESULTS AND DISCUSSION: A retrospective evaluation of clinical and epidemiological data of patients with a confirmed diagnosis of $\mathrm{AH}$ was made through an anatomopathological study between 1995 and 2015, followed by HC-UNICAMP. Through the evaluation of the histopathological characteristics of these tumors by two pathologists, they were classified into one of the four groups previously described. Twenty cases of $\mathrm{AH}$ were identified in the period, $90 \%$ in the female sex, 10 patients with oral contraceptive use (OAC). The most frequent clinical symptom was abdominal pain. The most comorbidities presented were systemic arterial hypertension $(n=7)$ and Diabetes Mellitus $(n=3) ; 60 \%$ of the cases had only one nodule. It was observed $35 \%$ of ruptures and 02 cases of malignancy, with 16 patients submitted to surgical resection. of the 14 patients operated on, nine presented AH of subtype 4 - without morphological alterations. The data found in our population sample are compatible with data found in the world literature. The correlation of the alterations seen in PA together with immunohistochemistry may suggest cases with unfavorable evolution. Conclusions: Oral contraceptives present an important risk factor. The most prevalent HA category is noninflammatory (exclusion diagnosis). There is no association between $\mathrm{AH}$ and smoking. In addition, we see that without maintaining the pattern of other studies, most patients, women of childbearing age.

\section{SYNTAX SCORE AS A PREDICTOR FOR POST-OPERATIVE COMPLICATIONS IN} CORONARY ARTERY BYPASS GRAFT SURGERY.

Nicolas Pedrosa Oleinik, João M Morosov, Isabela G Inocencio, Arly Vargas, Carlos F R Lavagnoli, Elaine S B Oliveira, Karlos A S Vilarinho, Pedro P M Oliveira, Orlando Petrucci, Lindemberg da Mota Silveira Filho.

Syntax Score (SS) was developed to analyze complexity of coronary vasculature and to aid in select better target patients for percutaneous treatment or coronary artery bypass grafts (CABC). The aim of this study is to verify the relation between SS, EuroSCORE risk predictor index and postoperative complications of CABG surgery. Between 2014 and 2016, 212 medical records from single CABC patients operated at Unicamp university hospital were retrospectively analyzed. The mean age was 62 years, $69.88 \%$ were male, $83.9 \%$ were hypertensive, $\mathrm{X} \%$ diabetic. SS was $41.36+/-13.74$ ), and EurOSCORE predicted a risk of $3.77+/-1.6 \%$. The hospital mortality observed was $4.18 \%$ and the total occurrence of complications was $30.76 \%$, most frequently atrial fibrillation, infection and acute kidney injury, which was present in $20.5 \%$. We conclude that in a series of CABC patients with a very high SS level, the morbidity rate was acceptable and mortality was consistently predicted by the EuroSCORE index.

07. MANAGEMENT OF INTRACRANIAL HEMORRHAGE IN PREGNANCY: REVIEW OF THE LITERATURE.

Isabela Veiga Daud, Pedro Boldrini Rezende Correa

INTRODUCTION: Intracranial hemorrhage (ICH) in pregnancy, although it has a prevalence of $0.01 \%$ to $0.05 \%,(1,2,3)$ presents a high rate of maternal and fetal mortality. Its most common causes in pregnancy are ruptured cerebral aneurysms, arteriovenous malformations (AVM), and preeclampsia/eclampsia. The risk of ICH in pregnancy is 5.6 times higher, due to hemodynamic, vascular and hormonal physiological changes. (4) Limitations of the imaging diagnosis, coupled with the specifications of the surgical management of the mother and the fetus, make the neurosurgical intervention in pregnancy a therapeutic challenge. (5) METHODOLOGY: We reviewed the main articles dated between 1990 and 2018. From this, we intend to explain the main causes of HIC, its incidence and risk factors in pregnancy; justify the propensity of $\mathrm{ICH}$ in pregnant women; discuss the different neurosurgical interventions and evaluate their complications and particularities during pregnancy. RESULTS: Pregnancy is characterized by physiological changes that favor the appearance and aggravation of cerebrovascular pathologies, such as HIC. Preeclampsia /eclampsia are related to ICH because they cause systemic spasms of small arteries and damage the endothelial cells. (6) The relationship between pregnancy and aneurysm and AVMs is poorly studied, but it is suspected that estrogen, cardiac output and volemia increase are involved. (4) Once the diagnosis is made, specific precautions are required in the management, such as choosing the anesthetic, adequate position in the surgical table, hyperventilation therapy, pressure control, irradiation exposure care and non-use of certain medicinal products. In the case of an aneurysm rupture one may make microsurgical clipping or endovascular coiling. In cases of AVM, when indicated, microsurgical excision is performed, whereas in preeclampsia/eclampsia the treatment is clinical. (7) CONCLUSION: ICH, despite being an uncommon pathology, presents high risks of maternal and fetal mortality. Early diagnosis and proper management at the right time are essential measures.

\section{EVOLUTIVE ANALYSIS OF A 20 YEAR PANCREAS TRANSPLANT PROGRAM.}

Tiago Cenzini de Miranda, Marcelo Perosa de Miranda, Gabriela Tomaz Martinho, Alessandro Silvestre, Nadia Mie Taira

There is a worldwide trend towards a reduction in the annual number of pancreas transplants (PT). In Brazil, only two centers have performed more than 20 PT per year. For this reason, the analysis of a large Brazilian series is of major importance.We analyzed 731 PT in a 20-year period, divided into 3 groups: ERA 1: PT performed between 1996 and 2003, ERA 2: from 2004 to 2009, ERA 3: from 2010 to 2016. Overall, 398 simultaneous pancreas-kidney (SPK) and 333 solitary PT (205 pancreas after kidney-PAK and 128 PT alonePTA) were performed. The distribution of PT categories showed a reduction of SPK and increase of PAK from Era 1 through Era 3 ( $p<0.001$ ). Among SPK, there was a lower number of preemptive transplant, from $26.6 \%$ in Era 1 to $4.76 \%$ in $\operatorname{Era}_{3}(\mathrm{p}<0.001)$ and the use of depleting antibody induction therapy increased from $53.7 \%$ to $88.9 \%(p<0.001)$. There was a drastic reduction in the use of bladder drainage from $61.7 \%$ to 0 at Era $3(p<0.001)$. Among solitary PT, the age of pancreas donors decreased over time (24.8 in Era $1 \mathrm{X}$ 20.9 years in Era 3, p<0.001) and there was a decrease in PTA $(62.5 \% \times 22.1 \%)$ and increase in PAK $(37.5 \% \times 77.8 \%, p<0.001)$. There was no significant difference in 1-year patient and graft survival over the Eras in both SPK and solitary PT. After 20 years of program, there was a decrease in the number of SPK and increase in PAKs. For SPK, most recipients are now transplanted on dialysis and receive induction therapy. For solitary PT, the number of PTA has decreased and younger donors were selected over Eras. There was a change in the PT technique, migrating from systemic-bladder drainage to portal- enteric (duodenal) in most PT. Patient and graft survivals remained similar throughout the analyzed periods.

\section{DRUGS USED IN PACEMAKER IMPLANT SURGERY.}

Carolina Kath Lucca, Andréia Cristina Tassi, Pedro Augusto Mortatti Ladeira, Vitor José Carvallo de Almeida, Felipe Castioni

INTRODUCTION: Artificial cardiac pacemakers are essential devices for the treatment of arrhythmias, for the correction of the natural electrical impulse of the heart and to promote control of the heart. Cardiac pacemaker implant surgeries have been increasing every year. The technology involving the devices, the drugs used and the surgical preparations are of extreme importance for a good prognosis and reduction of the complications. OBJECTIVE: To identify the drugs used in the management of artificial pacemaker implantation surgery, exposing their clinical and physiological characteristics, exemplifying the effect and action of the respective drugs, as well as their adverse effects, possible interactions and the correct dosage. It is sought to differentiate the drugs and to expose which are the most suitable and used in each case. METHODOLOGY: The information used to carry out this work was based on a bibliographical review of recent indexed scientific publications in the UpToDate, Scielo and Lilacs search sites. REVIEW: The cardiac pacemaker may be used on different occasions, therefore, it is of vital importance to the way that each drug is administered in relation to its purpose, dosage and dose both before and during surgery. After implantation, which may be through the types: endocardial, transvenous, mediastinoscopy and epicardial, it is necessary a follow-up for the whole life, effecting the ideal rhythmic excitation of the organ. CONCLUSION: Correct preoperative management of medications requires a constant exercise of clinical research, since it is very difficult to memorize the myriad interactions and complications related to drugs used. Therefore, it is very important to know the exceptions, since there may be severe anesthetic-surgical complications related to the inadequate management of 
the drugs in the preoperative period. Abrupt and profound changes in the usual medication during preparation for surgery should be avoided to the fullest extent since the decompensation of the underlying disease can be much more ominous than the maintenance of the usual medication.

10. CONGENITAL MEGACOLON: MORFOFUNCTIONAL REMAININGS ON RAT COLON AFTER BENZALKONIUM CHLORIDE TREATMENT.

Caroline Judeikis, Joaquim Murray Bustorff-Silva, Márcia Alessandra Cavalaro Pereira da Silva

Hirschsprung's disease is characterized by colonic aganglionosis, affecting mainly newborns. The current treatment of this pathology is surgical, but several studies are underway to develop less invasive methods that could cause less comorbidities. These studies use congenital megacolon models, one of which uses benzalkonium chloride to cause neurogenic ablation. The objective of this study is to demonstrate the effectiveness of aganglionosis provided by this drug by describing the remaining cells after denervation in order to optimize studies that use this model later.

11. CURRENT PERSPECTIVES OF SURGICAL THERAPEUTIC MANAGEMENT AND PROGNOSIS IN SYNDROME OF TREPHINED - BIBLIOGRAPHIC REVIEW.

Mateus Gomes Pereira, Tainá Momesso Lima, Gabriel Rocha Sanches, Nagila Emmanoele Bernardo da Silva

INTRODUCTION: Trephine syndrome (ST) is a complication of craniectomy, resulting in important neurological dysfunctions such as convulsion, severe headache, motor, cognitive and language deficit. ST arises from an emergency decompression (CD) craniectomy due to an increase in intracranial hypertension and Cranioencephalic Trauma, and cranioplasty is currently recommended as a treatment. OBJECTIVES: To present clinical and prognostic aspects of this neurological complication in the different types of bioprothesis in cranioplasty. METHOD: Bibliographic review of the clinical, therapeutic and prognostic data of ST in the population, in data indexed in MEDLINE, PubMed, SCIELO, using as criterion of selection of articles the language and year of publication, preferably Portuguese or English. Discussion: It is postulated that after the CD a set of forces begins to act in opposition: intracranial forces (CSF pressure, blood perfusion) and extracranial (atmospheric pressure, cutaneous flap weight). It is usually expected up to 2 years after the $C D$ for a cranioplasty. Autologous bone graft is the first choice, except when the size of the defect makes this option unfeasible. However, new methods suggest that using alloplastic materials, such as polymethyl methacrylate (PMMA), provide lower infection rates associated with cranioplasty at 5 to 8 weeks post CD. In addition, the use of alloplastics for surgery is also a method that replaces autologous bone in cases of peritoneal CSF. RESULTS: Autologous bone shows a lower risk of infection and extrusion. Alloplastic materials are the alternative in the absence of bone bioprothesis, mainly PMMA, however, it presents higher costs and risks of infections CONCLUSION: ST, although described in 1939 , still has questions about its pathophysiological mechanism and better therapeutic behavior. Therefore, the choice of surgical material should be discussed with the neurosurgery team individually.

\section{ARTIFICIAL CARDIAC PACEMAKER}

Andréia Cristina Tassi, Vitor José Carvallo de Almeida, Carolina Kath Lucca, Felipe Castioni, Pedro Augusto Mortatti Ladeira

In 1930, cardiologist Albert Hyman was responsible for the invention of the artificial cardiac pacemaker, which has since undergone changes with the advancement of technology, in search of more lasting problems. The aim of this study is to analyze the recent scientific publications about the creation evolution and methods of using the artificial pacemaker, as well as current epidemiology. The information used in this work was based on the bibliographical review of recent indexed scientific publications in Pubmed scielo and Lilacs. The cardiac pacemaker can be used in different occasions, characterizing the method created in the execution of surgeries that involve the artificial regulation of the adequate cardiac cycle. After implantation, which can be through the types: endocardial, transvenous, mediastinocospia and epicardial, the device works by regulating the transmission of the cardiac impulse, in patients who have sinoatrial or atrioventricular blocks, effecting the ideal rhythmic excitation of the organ. Artificial Cardiac Pacemakers are multiprogrammable electronic stimulation devices capable of replacing electrical impulses and / or ectopic rhythms to obtain cardiac electrical activity, as physiologically as possible. Thus, the artificial pacemaker replaces the natural electrical system of the heart which, under normal conditions, works with adequate regularity and frequency and responds according to the needs of the human body, such as rhythmic insufficiency.

\section{BREAKTHROUGH OF THORACIC DUCT IN LOW KINETIC TRAUMA:} DIAGNOSIS AND TREATMENT.

Gabriella Sapia Castilho, Carolina Gawlinski Franchi, Fernanda Americano Freitas Silva, Fernanda de Castro Nascimento, José Eduardo Cruz Cardoso

Chylothorax is the accumulation of the chylous in the thoracic, pleural or pericardial cavity, which can be formed either by the obstruction or difficulty of flow of the same, or by the laceration of the thoracic duct in a possible trauma. The symptoms are related to the presence of fluid in the thoracic cavity and are non-specific, which justifies the difficulty of diagnosis. The etiology of chylothorax can be divided into non-traumatic and traumatic ones, among which the low kinetic stress is highlighted. The diagnosis of traumatic chylothorax is essentially laboratory, since the clinical and imaging tests are not sufficient to confirm it. Given the above, the main objective of this report is to attenuate the importance of differential diagnosis and to expose the advantages of conservative treatment ove surgery in cases of rupture of the thoracic duct. The case of a 63-year-old male patient with a diagnosis of ruptured thoracic duct due to fracture of $\mathrm{T} 12$ is described here. After treatment with prolonged parenteral nutrition, with weekly supplementation of lipids and fat-soluble vitamins, the patient evolved with natural healing of the duct within 15 days of hospitalization and was discharged, thus evidencing the use of medium-chain triglycerides in a hypolipidic diet as main therapeutic method.

14. ULTRASSONOGRAPHY IN URGENCY AND EMERGENCY: A GUIDE FOR USE THE DIAGNOSTIC METHOD.

Andréia Cristina Tassi, Felipe Castioni, Fernando Pereira Vanni, Gabriella Sapia Castilho, Pedro Augusto Mortatti Ladeira.

The history of Ultrasonography (US) dates back to the early 19th century when the physical principles that form the foundation of this imaging method have been discovered. Since his appearance, has gained an increasingly important role in patient assessment, as an extension of the physical examination. Thus, the technical advances of the US diagnostic have been very accessible to the doctor as an imaging method to assess emergency situations and traumatic and non-traumatic emergencies. The objective of this work is to systematize the methodology of the urgency and emergency protocol and to adjust the use of ultrasound, as well as guiding doctors in the emergency room, as a route of guidelines for requesting urgency and emergency exams, thus increasing the quality of medical practice and offering greater safety in performing procedures. The information used to carry out this work was based on a bibliographical review of scientific publications recently indexed in search sites: UpToDate, Scielo and Lilacs. The US is a medical technique, used to reproduce realtime dynamic images of internal organs, tissues, vascular network and blood flow, assisting, complementing and interacting with other medical specialties; Presenting, therefore, vital importance during the different situations found in the urgency and emergency. The use of US in urgency and emergency, especially when evaluated by a trained doctor, helps decisively in the diagnosis and determination of the conduct for the critical patient. The theoretical and practical qualification and the adequate training are fundamental foundations in the implementation of this tool, offering the best treatment to the patients who need US.

15. THORACIC AORTIC AND THORACOABDOMINAL AORTA ANEURYSMS TREATED WITH CONVENTIONAL SURGERY: A RETROSPECTIVE STUDY IN THE UNIVERSITY SERVICE.

Naimy Boutros Carcia, Pedro Paulo Martins de Oliveira.

OVERALL OBJECTIVE: to identify the main comorbidities, intraoperative therapies and postoperative outcomes of patients with thoracic or thoracoabdominal aortic aneurysms who underwent conventional surgery at the Unicamp Clinical Hospital in the last seven years, relating them to each other and to data from the literature. SPECIFIC OBJECTIVES: to identify the relevant individual factors associated with the development of aneurysms and intra and postoperative complications; to identify intraoperative factors and therapies used and their association with intra- 
and postoperative complications, as well as the mortality of the procedure. METHODS: a retrospective study was carried out in the charts of patients treated at the Cardiac Surgery Service of the State University of Campinas (HC UNICAMP) between 2011 and 2017, with thoracic or thoracoabdominal aortic aneurysms submitted to conventional surgical treatment. INCLUSION: the medical records of all patients with thoracic or thoracoabdominal aortic aneurysm (older than 18 years of both sexes) submitted to conventional surgery in the period covered by the study were included. EXCLUSION: patients under 18 years and patients treated with endovascular surgery. DATA ANALYSIS: descriptive statistical analysis and use of GraphPad Prism 7.04 software for Windows with a $5 \%$ significance level. RESULTS: The medical records of 19 patients were analyzed; $95 \%$ were hypertensive, $37 \%$ were smokers, $21 \%$ were dyslipidemic and $31 \%$ had a history of previous aortic surgery. The main complications related to the procedure were infectious $(53 \%)$, pulmonary $(47 \%)$ and hemorrhagic $(21 \%)$, in addition to renal and cardiac / hemodynamic with a frequency of $16 \%$. These complications, according to the literature, may be related to intraoperative factors such as the use of extracorporeal circulation and transfusion of blood products, as well as with the comorbidities of the individuals. Surgeries of the thoracoabdominal aorta had $55 \%$ mortality, while those of the thoracic aorta had $20 \%$. The total mortality was $37 \%$. CONCLUSION: Comorbidities such as hypertension and smoking are relevant factors for the development of aortic aneurysms. Expressive complications related to the procedure may be associated with intraoperative and individual factors. However, although the data obtained were mostly consistent with the literature, no statistical significance was observed in this sample. Complementing with a study involving endovascular surgery and / or a larger sample, may be considerable to obtain other perspectives of the procedure in the university service, in addition to those already demonstrated with this research.

16. USE OF LEVONORGESTREL INTRAUTERINE CONTRACEPTIVE (SIU-LNG) BY WOMEN WITH SYSTEMIC LUPUS ERYTHEMATOSUS (SLE) WITHOUT ANTIPHOSPHOLIPID ANTIBODY SYNDROME (SAF).

Rafaella Colicigno Rebelo, Estephania Pignaton, Arlete Maria dos Santos Fernandes.

Systemic Lupus Erythematosus (SLE) is a chronic inflammatory autoimmune disease whose association with Antiphospholipid antibody syndrome (APS) may lead to increased disease activity and increased maternal and fetal morbidity/mortality. Effective contraception is necessary for women to be able to plan pregnancy at the most appropriate time. Pure progestogens are methods are the ones with the lowest use risks of use within this population, among them the levonorgestrel intrauterine system (IUS-LNG), which has a high contraceptive efficacy and a decreasing release rate over time. The goal of this study was to evaluate the health status and number of arterial/venous thromboembolic events (ATE/VTE) in women with SLE and SLE+APS using the IUS. It was a retrospective study with 46 women, 28 SLE and 18 SLE+APS, with data from medical records. We evaluated sociodemographic, obstetric gynecological variables, disease activity (Slice$2 \mathrm{~K}$ ) and damage (Slicc/ACR), and the number of ATE/VTE before and after IUS insertion. For analysis of the variables the Q-square, Fisher, and MannWhitney tests were used, and, to compare groups and times, the Generalized Estimating Equations were used. The significance level was of $5 \%$. The SLEDAI and SLICC scores were low pre-insertion and did not present an annual variation between the groups during 1-8 years of IUS use. The SLE + APS group presented higher BMI and ATE/VTE events before the insertion of the IUS; 15 women from the LES + SAF group had 37 ATE/VTE events, and two women from the SLE group had three events. After insertion of the IUSLNG 2/18 women with APS had three ATE. We interpret the results as a result of more serious disease activity of the SLE + APS group and not to the use of the contraceptive SIU-LNG. The selected IUS-LNG results did not contribute to worsen the health status of women with SLE + APS.

17. THE INADEQUATE COMPLIANCE OF THE CONSENT TERM IN GYNECOLOGICALOBSTETRIC SURGERIES: QUESTIONS OF TRANSPARENCY IN THE DOCTOR-PATIENT RELATIONSHIP.

Augusto Kerber Giacomoni, Eduardo Dambros, Eduardo Freitas de Oliveira, Letícia Oliveira de Menezes.

INTRODUCTION: The Informed Consent Form (ICF) informs and authorizes the patient or your legal guardian, from a legal and ethical point of view, to perform medical procedures. There is a standardization of completion of the Term according to norms of the Federal Council of Medicine - CFM - which has requirements that explain and clarify the methods, objectives, consequences and possible outcomes of medical procedures such as surgical procedures. OBJECTIVES: to analyze the quality of the filling of the ICF in surgeries of the area of Gynecology and Obstetrics - CO. METHODS: This is a descriptive, retrospective and cross-sectional study based on primary data obtained from a university hospital in the southern state of Rio Grande do Sul. It was evaluated whether the medical records signed by the patients and their filling quality in the surgical procedures in the CO area from April to July 2017. RESULTS: Out of a total of 365 gynecological or obstetrical surgical procedures, only 34 patients had the ICF filled out and signed in their medical records, which is equivalent to only $9.31 \%$ of the cases. None of the cases were completed in accordance with CFM standards. CONCLUSION: a more effective educational action is needed with physicians to reinforce the importance of transparency with the patient, properly filling out the Term of Consent, thus avoiding ethical and legal complications and aiming at the well being of the patient.

\section{INFLUENCE OF EDUCATIONAL ACTION OF MATERNAL BREASTFEEDING IN ADOLESCENT PUERPERS: NON-RANDOMIZED CLINICAL TEST.}

Renan Massao Nakamura, Fernanda Caranhani de Castro Surita.

INTRODUCTION: Breastfeeding (MA) is the first feeding practice, which is recommended only in the first six months of life and is supplemented with other foods after this time, up to two years or more. OBJECTIVES: To evaluate the effectiveness of educational action in prenatal follow-up on the intention to breastfeed and the performance of breastfeeding in the puerperium of adolescent mothers. METHOD: A non-randomized and open clinical study performed at the Hospital Dr. José Aristodemo Pinotti-CAISM / Unicamp. It has included primiparous adolescents with CAISM delivery, single fetus, alive and term. They were divided into two groups: Group 1) Puerperas who had prenatal care in the CAISM and participated in the educational activity of AM offered in the PNA (GI); Group 2) Puerperas that did not participate in this group (CSI). The collection occurred in two moments: during the stay of the puerpera and her child in the Joint Accommodation $(\mathrm{AC})$ and the first puerperal consultation in the outpatient clinic of Puerperal Review of Adolescents (RPA). At both times, the puerperae answered questions about AM and they and their newborns were observed during a feeding, filling out the "Breastfeeding Assessment Observation Form" of the Ministry of Health. Data analysis: Data wsd allocated in tables and graphs for descriptive statistical analysis (mean, standard deviation, absolute and relative frequency distribution). Open questions were categorized for the application of statistical analyzes. The level of significance adopted for the statistical tests was $5 \%$. For statistical analysis, we used the program Statistical Analysis System (SAS), version 9.4 for Windows. RESULTS: Of the 70 puerpera who were included, 27 were from intervention group $(\mathrm{Cl})$ and 43 from non-intervention group (CSI). At the first puerperal consultation 50 cases were reviewed, being 21 from $\mathrm{Cl}$ and 29 from CSI. Mean age was 16.7 years; the mean number of prenatal care consultation was 9.3 . At the first evaluation $81.5 \%$ from $\mathrm{Gl}$ and $78.3 \%$ from CSI presented good breastfeeding. A higher occurrence of inadequacy during breastfeeding was observed at the CSI group. From all the cases, seven resulted in weaning at the first puerperal consultation, all from CSI group. CONCLUSION: Group activities during prenatal care consultations have an important role encouraging breastfeeding, promoting educational activities to the adolescents. The puerpera feel prepared to breastfeed and there are less cases of early weaning. Other data collected in the present study also allow the elaboration of strategies to improve the group activities and strengthen subjects which the adolescents feel more difficulty during their first time breastfeeding.

19. VARIATIONS IN THE BLEEDING PATTERN IN LEVONORGESTREL INTRAUTERINE SYSTEM USERS AFTER THE FIRST CONSECUTIVE REPLACEMENT OF THE METHOD. Wittmann, B. Z.; Fernandes, A. S. M.

OBJECTIVE: To evaluate and compare the bleeding patterns presented by the users of the levonorgestrel intrauterine system (LNG-IUS, 52mg) in the end of the time of use and after the first consecutive change of method. SUBJECT AND METHOD: This was a retrospective study conducted at the Family Planning Clinic, University of Campinas, Brazil, with data collected from medical records. The variables were sociodemographics, obstetric history, time of use of the first and second IUS (IUS-1 and IUS-2), and the bleeding pattern presented in the reference period (RP) of 90 days before the change of the IUS- 1 and in the last medical appointment in use of the IUS-2. The bleeding pattern was classified in five categories: amenorrhea (lack of 
bleeding); spotting/scanty bleeding (any vaginal bleeding not enough to require protection or <2 episodes of spotting/bleeding); regular (3 episodes of spotting/bleeding with regular periods) and irregular (3-5 episodes of spotting/bleeding with variation between episodes $>20$ days) or increased (for at least one episode of referred bleeding as abundant or bleeding lasting $>8$ days). For statistical analysis, the two latest categories made up a single pattern called irregular/increased bleeding due to the small number of women presenting them. We used the qui-squared test, Mann-Wittney and KruskalWallis followed by the post-hoc of Dunn to identification of variables with association. The McNemar test was used to compare the bleeding patterns at the two moments and the KM curve to estimate the time of use of the method. RESULTS: We evaluated 301 women with mean age $( \pm$ SD, standard deviation) at SIU-1 insertion was $32( \pm$ SD 6.1) years, $75 \%$ of them opted for contraception and $25 \%$ for abnormal bleeding treatment. The mean time of use of the SIU-1 was 68.9 ( \pm 16.8) and the SIU-2 was 20.3 $( \pm 16.7)$ months, no differences in time of use or continuation rates between groups. At the end of the use of the SIU-1 and at the last consultation in use of the SIU-2, they presented amenorrhea $43.8 \%$ and $50.8 \%$; spotting / scanty bleeding $23.9 \%$ and $28.5 \%$; regular bleeding $25 \%$ and $15.5 \%$; and irregular / increased 9.3 and $5 \%$, respectively. The total time of use of the method was higher in women with amenorrhea pattern than those with irregular / increased and regular patterns. There was significant variation between the bleeding patterns presented in the two evaluated moments. CONCLUSION: Bleeding patterns with a lower flow/frequency increased and regular and irregular/increased bleeding patterns decreased during the use of the second device; however, changes also occurred with the replacement of lower flow/frequency patterns for the regular or irregular/increased bleeding patterns, which possibly does not express the decrease in the contraceptive efficacy of the method.

DIFFERENCES BETWEEN SEXUAL BEHAVIOR, KNWOLEDGE ABOUT HPV AND VACCINE AND RATE OF VACCINATION IN STUDENTS OF A PUBLIC UNIVERSITY.

Biselli-Monteiro, M, Derchain, S, Ferracini, AC, Sarian, LO

INTRODUCTION: Human Pappilomavirus (HPV) infeccion is the worlds most prevalente sexual infeccion and is related to the appearance of genital warts and various canceres in men and women. Many studies have shown lack of knowledge about HPV infection and consequences among young adults and low vaccination rate worldwide. MATERIALS AND METHODS: A first questionnaire, with questions about sociodemographic aspects, sexual background, knowledge about the HPV and its vaccine, vaccination status and desire to indicate the vaccination to young adults in future practice was applied to 492 students of medicine, farmacy, fonoaudiology, nursing school and physical education. In a second moment, another questionnaire was applied to 233 students of medicine and farmacy, to evaluate the rate of vaccination after participation on the first part of the study. For the analyses were used $\chi 2, \chi 2$ for trends and $t$-test. RESULTS: a total of $47 \%$ of the first year and $13 \%$ of forth year women were not sexually active vs $11 \%$ of men from first and fourth year. The use of condom was lower in women $(56 \%)$ than in men ( $96 \%)$. $90 \%$ of women had gone to the gynecologist vs $26 \%$ of men to the urologist. The knowledge about HPV was higher among women. More than $83 \%$ of women and $66 \%$ of men knew that HPV can cause cervical cancer. Less than $30 \%$ of all the students knew that the HPV can cause other cancers, and only $48 \%$ of women and $38 \%$ of men knew that it can cause genital warts. When comparing first and fourth year, there was a higher rate of correct answers among forth year students about the sexual transmition of HPV, higher incidence between 15 and 25 years and possibility of assintomatic infeccion. The knowledge of the students incrised when comparing physical education, farmacy/fonoaudiology, nursing schoo and medicine. 233 students answered the second questionnaire, among them, 39 women and 15 men had received the vaccine previor to the first part of the study. An addicional 34 women and 10 men affirmed that had received the vaccine after participating on the first questionnaire. CONCLUSION: The knowledge was higher in women, but men declared to use more condom. Almost half of the women start university without having started sexual life. The rate of vaccinated students was low, and those who participated on the first part of the research were more likely to get vaccinated. The admission on superior education seems like a good time to estimulate students' knowledge about the HPV and their vaccination. PELVIC ORGAN PROLAPSE.
Carolina Petraites Rosati, Bruna Dias Pacheco, Cristiane Regina de Barros.

INTRODUCTION: Pelvic organ prolapse (PLS) is a relatively common occurrence and, although it does not pose a risk to life, it can adversely affect a woman's quality of life. Because it is more common in women with advanced age the conservative treatment is being more desired, since the surgical treatment may have contraindications clinical. OBJECTIVE: to analyze the published studies regarding the conservative treatments of POP through physiotherapy, as well as factors described as success in the treatment of POP. METHODS: Bibliographic review using the Scientific Electronic Library Online (SciELO), Lilacs and Bireme with terms " Pelvic physiotherapy, pelvic floor and prolapse " published from 2011 to 2018. RESULTS: Studies show that treatment with physical therapy for POP obtained good results, authors have suggested that more studies be done on this form of treatment so that more people adopt this approach for treatment. CONCLUSION: Physiotherapy treatment is a great treatment option for women affected by POP because it is not as invasive as surgery and also easier and simpler to apply.

\section{GESTATIONAL ABNORMALITIES CAUSED BY DRUG ABUSE.}

Fernanda Folla Pompeu Marques, Mércia Tancredo Toledo, Emanuela Yumi Fugisawa de Mello, Tiago Cenzini de Miranda

Pregnancy is a fundamental period of fetal growth and development, therefore need great medical care with patients. Many are the teratogenic agents, in the class of chemical, persistent use of tobacco, marijuana and cocaine is addressed during pregnancy. Motivated by a large number of drug users patients, the authors aimed to revise the literature on the changes, fetal and pregnant women, arising from the use of tobacco, marijuana and cocaine. Critical review of publications in MEDLINE, SciELO and Coogle scholar, between 2007 to 2017 in English and Portuguese was made. SUS guidelines and manuals have also been reviewed. After acquiring knowledge about the effects of the main psychoactive drugs used during pregnancy, an educational infographic was created and delivered to pregnant women in a Primary Health Care Center of Sorocaba. Drug abuse is not an exclusive problem of Brazil and women of childbearing age are mostly in the use. Cannabis sativa is the illicit drug of increased abuse among pregnant women, affecting the development of the nervous system with bad formation of the neural tube and possible anencephalies. Low birth weight and increased likelihood of use of the neonatal intensive therapy unit are also mentioned. Cocaine has a soluble character and crosses the placental barrier by simple diffusion. It causes fetal deformation, premature detachment of placenta, increased maternal blood pressure and decreased fetal circulation. Smoking can cause fetal hypoxia, raising the risk of cerebral infarction in the neonate, prematurity, abort, pre-detachment of the placenta and less weight of the neonate at birth are also cited. In the face of scarce studies and government programs there is a need for greate attention on this public health problem in order to empower the health teams to provide information to pregnant women during prenatal care.

\section{PAGET DISEASE OF THE BREAST: A DESCRIPTION OF THE CASUISTRY OF THE CENTER FOR INTEGRAL ATTENTION TO WOMEN'S HEALTH (CAISM- UNICAMP)}

Amanda Bispo Velasco, Mariana Dias Vasconcelos, Cássio Cardoso Filho.

Paget disease is a subtype of rare mammary cancer and represents $0.5 \%$ a $5 \%$ of global breast cancer. This condition presents with eczema of the nipple and histologically with malignancy cells in the epidermis called Paget cells. It is associated with an underlying breast carcinoma in more than $90 \%$. There are yet a lot of doubts and paradoxes about the best treatment and prognosis because your histogenesis e risk factors are not settled down. Therefore this study aims to describe in this population the epidemiological and clinical characteristics of their disease, as well as the options of treatment. The objective of this study is calculate the frequency and describe clinical characteristics, mammographic features, and histological features, as well as, forms of surgical treatment and prognosis of the patients with Paget disease of the nipple. Subjects and methods: patients with Paget disease of the nipple undergoing treatment in the Center for Integral Attention to Women's Health (CAISM) will be evaluated in describe in a descriptive analytical study from 2002 to 2017 , by analyzing medical records in accordance with the Ethics and Research Committee rules. 
24. HEMORRHAGIC COMPLICATIONS IN THE PREGNANCY-PUERPERAL CYCLE IN PREGNANT WOMEN WITH VON WILLEBRAND.

Nagila Emmanoele Bernardo da Silva, Gabriel Ramon Matavelli Casseb, Rafaela Seraphim Frare

BACKGOUND: von Willebrand disease (VWD) is a hemorrhagic disorder originating from an autosomal dominant genetic inheritance pattern with a prevalence of $1 \%$ of the world population. The von Willebrand Factor (VWF) is a multimeric endothelial protein that acts in the platelet adhesion phase and also as a coagulation factor VIII carrier. Although it is a disease that affects both men and women, the symptomatology is more severe in women, due to hemorrhagic complications with menstrual and gestational cycles. OBJECTIVE: A literature review on the risks and gestational complications hemorrhagic of patients with VWD. METHODS: Literature review of scientific articles in sources such as Lilacs, PubMed, Elsevier and Scielo. RESULTS: A higher incidence of hemorrhagic events was observed in pregnant women with VWD in the first trimester, and a decrease in these events after the second trimester. In addition, it was found that the delivery and puerperium are the most critical periods of these pregnancies. DISCUSSION: Studies has indicate the incidence of hemorrhagic conditions in the first trimester of pregnancy with VWD, followed by a decrease in the probability of these events from the second trimester. Furthermore, the literature emphasizes the importance of adequate gestational prophylaxis for the prevention of hemorrhagic complications at delivery and in the puerperal period. CONCLUSION: Pregnant women with VWD require specialized medical assistance during the pregnancy-puerperal cycle with the intention of minimizing the occurrence of hemorrhagic conditions and their eventual complications.

\section{MAYER-ROKITANSKY-KÜSTER-HAUSER SYNDROME: THERAPY AND} UTERINE TRANSPLANTATION.

Luana Monte, Attílio Brisighelli Neto, Mariana Cristina Ferreira da Silva, Ana Clara Charantola Beloni, Ana Beatriz Charantola Belon.

INTRODUCTION: Mayer-Rokitansky-Küster-Hauser syndrome (MRKH) results from congenital malformation of Müllerian ducts, involving abnormalities from the reproductive system to the renal, bone and cardiac systems. It is characterized by modifications that compromise sexual life and female fertility, necessitating therapeutic methods, such as uterine transplantation, an innovative but experimental possibility. OBJECTIVE: To report the possible therapies employed and to address uterine transplantation as a possibility of gestation and promotion of well-being in patients with MRKH. METHODOLOGY: A review of the literature on MEDLINE, PubMed and LILACS on the topic of Mayer-Rokitansky-Küster-Hauser Syndrome of the last 15 years. RESULTS: According to data, the treatment begins with non-surgical procedures, with use of vaginal dilators, presenting $90-96 \%$ efficacy. The surgical correction consists in the creation of a neovagina, but does no solve the inability to gesta. Uterine transplantation, although recent and experimental, has shown promising results, with the birth of healthy children, providing hope to women with the syndrome. DISCUSSION: Due to the late diagnosis, changes from the syndrome compromise the woman's quality of life. Conventional therapy provides a normal sex life for the patient. Despite the functional ovaries, gestation is only possible with uterine transplantation associated with in vitro fertilization, feasible since 2014, after the birth of a child in Sweden. The procedure, still experimental, requires care, both for the donor and for the recipient. However, some aspects still need to be studied, such as the number of viable pregnancies, the psychological evaluation of the patients and the long-term developmen of the babies. CONCLUSION: Despite advances in the therapeutic approach of this syndrome, the choice of procedure should be adapted to the needs, motivation and emotional maturity of each patient. Therefore, there is no best technique to intervene in the syndrome, but rather the best technique for each patient.

\section{INCIDENCE AND CHARACTERIZATION OF PRE-ECLAMPSIA IN A} REFERENCE SERVICE: PARTIAL RESULTS.

Christopher Cralcev, Leonardo Bernardes de Amaro, Juliana da Costa Santos, Marcos Marangoni Junior, Maria Laura Costa do Nascimento.

INTRODUCTION: Hypertensive syndromes are one of the main causes of maternal mortality and morbidity worldwide. Preeclampsia (PE) accounts for about 50,000 maternal deaths annually worldwide. PE is a multifactorial disease that occurs when there is impairment between the balance of pro angiogenic and anti-angiogenic factors in the trophoblast invasion, manifesting itself from the twentieth week of pregnancy in normotensive patients. Cases with a diagnosis of preeclampsia, especially preterm or with severity signs, should be transferred to a reference center. The Women's Hospital Prof. Dr. José Aristodemo Pinotti - CAISM / UNICAMP is a regiona reference in tertiary and quaternary complexity in the health care of women and the newborn, with an Obstetric and Neonatal Intensive Care Unit. OBJECTIVE: to evaluate the incidence of hypertensive disease and preeclampsia, maternal and perinatal outcomes, as well as the correct use of the International Code of Diseases (ICD) during hospitalization and discharge, among all the cases admitted to the CAISM / UNICAMP. MATERIAL AND METHOD: this is a cross-sectional study with a medical record review. Preliminary results with period of 4 months. Approval of the Research Ethics Committee \# 2,817,427. Data analysis was performed in SPSS software version 22. RESULTS: 1000 deliveries were evaluated within 04 months, of which $35.2 \%$ were from primigravidae pregnant women. The median of maternal age was 27 years and the number of vaginal deliveries computed $50.3 \%$, in contrast to $49.7 \%$ of cesarean deliveries. The incidence rate of hypertensive disease was $14.3 \%$ (including chronic hypertension $(\mathrm{CH})$, gestational hypertension $(\mathrm{CH})$ and $\mathrm{PE}$ ). There were 51 cases of $\mathrm{GH}, 44$ cases of $\mathrm{CH}$ and 83 cases of PE (incidence of $8.3 \%$ ). Of these, $73.5 \%$ had a severe manifestation of the disease, $7.2 \%$ had HELLP syndrome and $3.6 \%$ eclampsia. Considering the route of delivery among the cases of PE, there were $78.3 \%$ of cesarean deliveries, with $26.5 \%$ of induced deliveries. Among the cases of PE, more than half of the newborns were preterm (below 37 weeks) $(56.6 \%)$, and $30.1 \%$ were diagnosed with early PE (below 34 weeks). When evaluated the use of the ICD assigned in the hospitalization of the pregnant women who had $\mathrm{PE}$, it is possible to notice that approximately $28 \%$ of the cases received the incorrect identification; a higher value, approximately $57 \%$, of inappropriate use of the code occurred at discharge. CONCLUSION: the Women's Hospital, CAISM/UNICAMP, has an incidence of $8.3 \%$ of $\mathrm{PE}$, with a significant percentage of severity. The data obtained in this initial evaluation help to better understand the impact of PE on the obstetric care of a referral center. The end of the survey of the other obstetric care that took place in one year (an estimated number of more than 3,000 deliveries) and the detailing of the maternal and perinatal outcomes will provide subsidies for multidisciplinary team training, better case management, reducing maternal morbidity and perinatal complications, optimizing the assistance to pregnant women and the adequate computerized hospital registry.

\section{PLACENTA BIOBANK IN A REFERENCE MATERNITY: CHARACTERIZATION OF ZIKA VIRUS INFECTION.}

Japecanga, R.R.; Guida, J.P.; Nobrega, G.M.; Venceslau, E.M.; Tavares, A. A.; Proenca-Modena, J.L.; Costa, M.L.

Zika-unicamp network.

INTRODUCTION: Biobanks are organized collections of human biological material and associated information, stored for research, under institutional responsibility. The placenta represents the interface between the mother and the fetus. Abnormalities in this circuit are associated with the development of diseases and may help to understand the pathophysiology of conditions still poorly understood (such as ZIKA virus). OBJECTIVE: to implement a protocol for systematic collection of placentas in a high risk maternity, prioritizing cases of arboviruses and characterizing ZIKA virus infection, with maternal, perinatal and placental results. METHODS: Retrospective Cohort. Placental fragments from the different regions (amniotic membrane, chorionic plaque, villous tissue, basal plate and umbilical cord) were collected with tissue stored in a paraffin block (for histological evaluation) and frozen at $-800 \mathrm{C}$ (for extraction of RNA, DNA and protein). In addition, the maternal and fetal placental faces, placenta weight and volume were recorded. Data on demographic, clinical characteristics, maternal and fetal outcomes were also obtained by medical chart review. RNA extraction and detection of ZIKV by qRT-PCR from placental fragments using the TaqMan kit (Thermo Fisher). RESULTS: from May / 16 to July / 18, samples of 143 placentas were collected; of these, 82 were cases of preeclampsia ( 1 of them with ZIKV infection), 18 of arboviruses (ZIKV), 29 for other diseases and 15 were included as controls (no pathology). The gestational age of the suspected ZIKV samples collected, ranged from 34 to 40 weeks. The mean placentas weight was 512.94 g; the mean volume was $442.5 \mathrm{~mL}$. Among the 18 suspected / confirmed cases of ZIKV, 1 case is stil under evaluation, 6 of them had positive serum PCR for ZIKV; 13 were positive in the placenta. of these placental positive, 12 cases in chorionic villi, 6 cases in the basal plate, 6 cases in the amniotic membrane, 7 in chorionic plaque, and in 4 cases in the umbilical cord. In the morphological analysis 4 cases presented villitis or vasculopathy. CONCLUSION: Having an 
institutional biobank allows for quality resource of material. ZIKV can infect different regions of the placenta of naturally infected pregnant women. The ZIKV detection in placenta after several months of initial symptoms, and in mothers without ZIKV detection by qRT-PCR during acute phase, suggest that this tissue can be a site for viral persistence during pregnancy.

\section{A CASE SERIES ON THE USE OF LEVONORGESTREL 52 MG INTRAUTERINE} SYSTEM AFTER ORGAN TRANSPLANT.

Cassia R. T. Juliato, Paulo Stahlschmidt, Arlete Fernandes, Ilza Monteiro, Luis Bahamondes.

OBJECTIVE: To assess the clinical performance (pregnancy and occurrence of pelvic infection) of the levonorgestrel 52-mg intrauterine system (LNG IUS) in solid organ transplant recipients. STUDY DESIGN: We performed a retrospective chart review of all patients seen in the Family Planning Clinic at the University of Campinas Department of Obstetrics and Cynecology from 2007 to 2017 to identify women with solid organ transplantation using immunosupression who received an LNC IUS. Our routine clinical practice for such patients is to perform initial follow-up 40-60 days after placement and then every 12 months. We analyzed women's sociodemographic characteristics, duration of use of the LNG IUS, continuation and discontinuation reasons, and complications. RESULTS: of the 23 women evaluated, 21 had kidney transplantation and 2 had liver transplantation. One woman received an LNG IUS prior to solid organ implantation ( 1.7 years, renal transplant). The 22 other women received the LNG IUS a median of 6.3 years (interquartile [ $1025-75]$ range $1.7-15$ years). Seventeen ( $74 \%$ ) women primarily used the LNG IUS for contraception; the other 6 desired the LNC IUS for contraception and heavy menstrual bleeding (HMB) treatment. W observed no cases of pregnancy or pelvic infection. Over the median followup time of 49.3 months (IQ25-75 15-80 months), 7 women used the same IUS for more than 60 months and 8 women received a second IUS CONCLUSIONS: Due to the fact that no pregnancies or pelvic infections were observed in this case series, we concluded that the LNC IUS is an option fo transplanted women for contraception and for HMB treatment. Implications Women at reproductive age after solid organ transplantation needs contraception for long time and the LNG IUS is a safe and effective option.

29. FAMILY AND COMUNITY MEDICINE LEAGUE LIKE APLICATION TOOL OF NEW BRAZILIAN NATIONAL CURRICULAR GUIDELINES OF MEDICINE COURSE.

Heitor Basaglia Almagro, Flávio Pereira dos Santos Júnior, Fernanda Aparecida Novelli Sanfelice.

INTRODUTION: The Family and Comunity Medicine League (FCML) was inspired in the proposal of in force college medicine course, of support and incentive to general doctor formation, by the interest of founder members in the Family Health Program (FHP). OBJECTIVE: Present an extracurricular activities model turned to primary attention, suggest student interventions to stimulate the general doctor formation and emphasize the importance to attend the Brazilian territorial health needs exposed by New Brazilian National Curricular Guidelines (NBNCG). METHODOLOGY: The documentation (learning plane, year purposed activities schedule and intern regiment) has been requested for League opening by side of orientation of college academic league directory. After documentary analysis and approval, the league started its activities that lasted since 2016 Dezember to 2017 November. RESULTS: The NBNCG was contemplated by the principle of university tripod and the care of Brazilian territorial needs. DISCUSSION: The results manifest by a praticle and visible approach of NBNCG adequation, the student enlargement of knowledge in FCML, attention to brazilian territorial health needs and the academic practice with the possibility of knowledge dissemination, since this integration principle still does not occur. CONCLUSIONS: The Brazilian public health governed by assistencial model based in primary attention and the implantation of NBNCG open strong perspectives for the effectiveness participation of Family and Comunity Medicine and primary attention in curriculum of Brazilian grade programs. However this insertion still is a preamble that requires systematization and incentive, mainly by the criation of academic spaces like the academic leagues, that encourage the presence of Family and Comunity Doctors in its reality.

30. PREVALENCE OF VACCINATION IN ADULTS WITH HIV INFECTION FOLLOWED AT HOSPITAL OF CLINICS - UNICAMP.
Carolina Neves Bühl, Maria Rita Donalisio Cordeiro, Márcio Cristiano Melo, Flávia Moreno, Flávio Polillo.

The access to medical assistance and prevention measures increased HIV/AIDS patients' survival in Brazil. Vaccination may avoid infections that can lead to severe outcomes in immunodepressed patients. The objective of this study is to investigate the prevalence of immunizations indicated by the Ministry of Health, in the patients followed at the HIV/AIDS Outpatient Clinic of Hospital of Clinics - Unicamp. We analyzed 340 medical and immunization records of patients who attended the consultation in 2016. The variables studied were socio-demographic epidemiological, and clinical. Vaccination records showed an average of $50.5 \%$ coverage of Pneumo 23V, Diphtheria- Tetanus (51.6\%), Hepatitis B (54.2\%), Hepatitis A (7.5\%) Influenza (42.9\%), Meningoid C (44.9\%) vaccines. Lack of information about vaccination was higher among young ( $<39$ years) male patients, last CD4 «350 cells/ul. Higher prevalences of incomplete schemes were also identified primarily with the vaccine Meningoid $C$ and Influenza. Although patients may have received vaccine in other health services, lack of information in the medical records can compromise the integral care to the patients.

\section{THERAPEUTIC ITINERARIES OF PATIENTS WITH COLORECTAL CANCER ATTENDED AT THE HOSPITAL DE CLÍNICAS DA UNIVERSIDADE ESTADUAL DE CAMPINAS}

Seok Woo Shin

Cancer is one of the most prevalent diseases worldwide. Among the neoplasms, colorectal cancer stands out for its magnitude and severity, and for having methods of population screening that have an impact on the prognosis of the disease. Objectives of the study: Reconstitute and analyze the Therapeutic Itinerary ( $\mathrm{TI}$ ) of colorectal cancer patients attended at the Hospital de Clínicas da Unicamp, seeking to characterize the reasons for searching care, the health services sought, the facilities and/or access difficulties and the time intervals between diagnosis and treatment. Method: Qualitative research, with data collection through semi-structured interview and consultation of medical records. Thematic content analysis of 22 TIs was carried out. Results: The main symptom that motivated the search for assistance was bleeding in the feces. Only 3 patients had been screened for colorectal cancer. Although the Basic Health Unit was initially the most sought after service, the Tls configured a public-private mix of services, with the high complexity attention being assumed by the SUS. There was no regularity in the flow of patients through the service network and there were varied pathways and difficulties to access care. The interval between diagnosis and initiation of treatment ranged from 22 to 300 days, above the 60 days recommended by the Ministry of Health.

\section{SELF-STEEM, SPIRITUALITY AND QUALITY OF LIFE IN PATIENTS WITH LOWER LIMB ULCERATION.}

Ana Beatriz Nascimento Costa, Thuany Caroliny Coelho, Marcella Nora Maia, Sílvia Mara Tasso, Nathalie Bartelega Dominguetti.

INTRODUCTION: Lower limb ulcers are very frequent and of great medical and social importance, since they are extremely incapacitating and significantly affect the productivity and quality of life of the individuals, as well as determining significant expenditures for health services. In addition to the therapy associated with traditional medicine, it is common for patients to seek other therapeutic models based on their beliefs, placing their hope in the capacity to overcome their difficulties. METHODS: Individual, analytical, observational, transverse and uncontrolled. The sample was for convenience consisting of 42 patients, collected at the Assistance and Nursing Educational Centre (NAEENF) of the Samuel Libânio Clinical Hospital. An instrument containing sociodemographic data of the subjects who took part in the research was used to evaluate their self-esteem. Data was collected using the Rosenberg UNIFESP / EPM Self-esteem Scale and the WHOQOL-SRPB questionnaire, developed by the WHO (World Health Organization) to assess religiosity, spirituality and personal beliefs. RESULTS: The epidemiological profile of the research was traced as the majority of the people being male, under 65 years old, with complete elementary school, white skin, with no companion and retired. With the application of the tests, age presented statistical significance with fullness, spiritual strength, inner peace and self-esteem. Regarding gender, health status, schooling, marital status and skin color, none of the facets presented statistical significance. With regard to professional occupation, the inner peace facet presented statistical significance and in regard to origin, the fullness facet presented statistical significance. CONCLUSION: The age facet, 
when in advanced, negatively influences future perspectives, contributing to low self-esteem, lower spiritual strength, inner peace and fullness. Professional occupation contributes to better self-esteem by showing that people feel more valued and helpful. In relation to the fact that people have companions, our research was contrary to the literature, not contributing to soften the limitations and as a support in the difficulties of the everyday life.

\section{GEL OF BANANA PEEL AND THE QUALITY OF LIFE IN CHRONIC ULCER} CARRIERS.

Tamires Sousa dos Anjos, Myrian de Andrade Ferreira, Fabio Roberto Guido Júnior, Clarissa Fabianek, Adriana Rodrigues dos Anjos Mendonça, Dênia Amelia Novato, Castelli Von Atzingen.

INTRODUCION: The appearance of chronic wounds cutaneous is relatively common, mainly secondary to various health problems. Trying to prove the effectiveness of popular knowledge can generate good results at a lower cost, an example is banana, tropical fruit Brazil has great production. The coverages classified as herbal medicines are those that used in their composition medicinal plants in their different pharmaceutical forms, without the use of isolated active substances, although of vegetal origin. The research aimed to evaluate the quality of life in patients who have diabetic and venous wounds treated with ointment based on banana extract of the Musa sapientum species.OBJECTIVES: To evaluate the quality of life in patients with Chronic ulcers treated with gel based on banana extract Musa sapientum. METHODS: The questionnaire Ferrans and Powers, validated, for the wounds version, Beatriz Yamada, applied to patients who have accepted the use of the gel obtained by peeling green bananas for 4 weeks. Thirtysix people were interviewed and followed-up, 18 in the experimental group and 18 in the control group. RESULTS: The evaluations showed that the use of the gel does not affect the QOL of patients, $p=0.9165$ compared between the control groups, $p=0.7532$ between experimental, $p=0.8728$ between control and experiment before ep $=1,000$ between control and experiment after use. The gel did not affect the quality of life of the patients, being an advantage, since there was no loss with the new treatment. When a new proposal is made, you should think about the disease but also about the patient, their well-being and their adherence CONCLUSION: Wounds are chronic pathologies, difficult to treat and resolve, which greatly affects the QoL of the patients. That work will serve as a subsidy and encouragement for others to be developed in both the treatment and the patient's assessment usually part often overlooked, but very related.

\section{QUALITY OF LIFE AND PERCEPTIONS OF THE PATIENT SUBMITTED TO} HEMODIALYSIS.

Lucas Ribeiro Souza, Arícia Dutra Cardoso, Laryssa Rodrigues de Lima Trinchão, Luciana Bitencourt Carvalho, Silvia Mara Tasso.

INTRODUCTION: In the recent decades, chronic diseases have received greater attention from health professionals. The extent and complexity of the problems inherent to the experience of the chronicity of these diseases stimulates the analysis of patients' quality of life. METHODS: Analytical, observational, transversal, individual and uncontrolled study. It was estimated a population of 108 patients who underwent hemodialysis treatment at the renal replacement therapy service at Samuel Libânio Clinic Hospital in Pouso Alegre. Two instruments were used: initially, the sociodemographic protocol and then the specific Questionnaire (Renal Disease and Quality of Life - KDQOL-SF 1.3). RESULTS: Sex (Female 34,30\% and Male $65,70 \%$ ), Age group ( $18-30$ years old $10,20 \%, 31-60$ years $52,80 \%$, and > 61 years $37 \%$ ), salary ( 1 to 4 wages $77,80 \%$, 5 to 8 wages $8.30 \%$, 8 wages $0.90 \%$, and do not know $13 \%$ ) and Ethnicity (White $74.10 \%$, Black $22.20 \%$, and Others $3,70 \%$ ). We correlate the symptoms, disease effects, disease burden, professional role, cognitive functions, social interaction, sexual satisfaction, sleep, assistant team stimulation, personal satisfaction, physical functioning, pain, emotional well-being, and other factors. CONCLUSION: We conclude that the Quality of Life of the Patient submitted to Renal Replacement Therapy does not have income and color interference general care related to social history, such as unrestricted eating and / o control, non-exercise and alcohol/smoking interfere in a negative way. The severity of the disease presented by the patient and/or the various pathologies that he/she had influenced the statistical significance, meaning that, the more serious the patient's general situation, the more negatively influenced his overall condition in the treatment. With advancing age, the effects caused by chronic renal failure and/or effects elicited by renal replacement therapy were more exacerbated.

\section{VOLUNTARY, INVOLUNTARY AND COMPULSORY HOSPITALIZATION OF CHEMICAL DEPENDENTS.}

Arthur Manzani Fernandes, Thaís Cristina Faria Pacheco, Nayane Almeida Luiz, Hadassa Louback Paranhos, Eduardo Henrique Teixeira.

Chemical dependency has been treated as a matter of international problem, motivating several discussions among which therapeutic alternatives are highlighted, especially the modalities against patient's will - involuntary and compulsory admissions - creating debates that involve clinical, legal, ethical and social perspectives. The objective of this article is to make a critical review of the scientific literature about different approaches in treatment of drug dependence and its clinical impacts. A literature review was performed by electronic research in Pubmed, Bireme, Medline, and Scielo databases finding 39 Portuguese and English articles between 2002 and 2017. The themes presented were: differences in the therapeutic approach among countries, diagnoses, type of admission and impact, approach in adolescents, legal and ethical aspects. It has been pointed out that involuntary and compulsory psychiatric treatment has been a source of debates and has undergone updates through new legislation in several countries; there are differences in the profile of patients according to the hospitalization modality adopted in each case; chemical dependency has been shown to be one of the main diagnoses associated with psychiatric admission and there is evidence that even treatments against patient's will shows to be effective; the interlocution between health and law areas is fundamental; there is still an important shortage of studies in this area.

\section{CLINICAL AND EPIDEMIOLOGICAL EVALUATION OF PATIENTS WITH NEW DIAGNOSTICS OF HIV INFECTION BETWEEN 2013 AND 2016.}

Tamiris Ricci Camisa Nova, Thaís Cristina Faria Pacheco, Tayrine Borges Barbieri, Camila Cristina Salazar Torres, Elisa Donalísio Teixeira Mendes.

The acquired immunodeficiency syndrome (AIDS) has a featured between the sexually transmitted infections, because of its gravity, prevalence and for still be until now, incurable. The objective of this paper is to describe the profile of the population with a new HIV diagnostics in the PucCampinas Hospital. It is about a cross-sectional descriptive-retrospective study with a quantitative approach which data bases were extracted from Puc Campinas Hospital medical reports, selecting new cases diagnostics of HIV infection between January of 2013 and December of 2016. Were analyzed 185 medical reports, the year of 2013 had the most number of notifications. Most of the patients were on the age range of 30 to 39 years old, natives from Campinas, had brown color, with more than 8 years of study and 5.37 $\%$ were pregnant woman. The proportion of men:woman was $2,5: 1$ and $23 \%$ of man declared the mode of explosion as homosexual. In the diagnostic, $33,87 \%$ had an opportunist disease, the most commonwerepneumocystose $(10.85 \%)$ and neurotoxoplasmosis $(4.3 \%)$. The initial average of CD4 was $328.04 \mathrm{un} / \mathrm{ml}$ and the viral load (VL) 162614.8 copies $/ \mathrm{ml}$. In the diagnostic, $6 \%$ had undetectable VL and, after a year, $32.7 \%$. Of the sample, $13.9 \%$ died. It was notice a decrease of incidence in the Southeast, a data also found in your study, moreover a increase of the proportion between men:woman was in the younger age range was observed. The homosexual exposure between man in your study $(23 \%)$ is smaller than the one found in the state of São Paulo (44.5\%). The diagnostic was late in most of the cases, detecting patients already immunologically vulnerable, demonstrating the failure of early diagnosis and reflecting in the outcomes, still unfavorable in your region. The adherence to treatment occurred only in $32.7 \%$ if we considered the undetectable VL, suggesting the vulnerability in the appropriate medical accompaniment in this population.

\section{ANIMAL HOARDING - INTERVENTION OF FAMILY HEALTH STRATEGY IN ARARAQUARA - SP.}

Gabriella Sapia Castilho, Andreia Cristina Tassi, Phelipe Antonio Calixto, Antonio Raí Trentim.

Animal Hoarding Disorder includes a psychiatric disorder with symptoms related to the need to collect animals and the difficulty in disposing of them. Most of the time, the compulsion to accumulate damages several aspects of daily life, such as the social and occupational scope, making it difficult to live with these individuals. We describe here the case of a female patient, 87 years old, accumulator of Animals, with ObsessiveCompulsive Disorder, bedridden, with limb ulcers, perineal ulcers and poor hygiene, requiring the intervention of the health team at home. The main objective of this work is 
to report the joint care plan between health unit "Jardim Brasil", Araraquara Environment Department and family members for health promotion. After intervention, the patient was persuaded to improve her hygiene and initiate treatment via topical and oral antimicrobials for perineal lesions and antipsychotic in low doses for delusions, and died from natural causes. It is concluded that complex cases such as Hoarding Animal require actions involving various levels of the system and intersectoral cooperation, evidencing the importance of the health team for its competence in coordination of care, longitudinality and bond, promoting quality of life for the patient and her Family.

\section{NUTRISUS DEVELOPMENT AND IMPLEMENTATION: AN ANALYSIS OF PUBLIC POLICY.}

Natália Pereira Silva, Julicristie Machado de Oliveira.

Understanding how the strategy is practiced (NutrisuS), by who it is carried out, how it is done and whether it is actually effective or not, was the aim of the first phase of this research. Thus, several materials related to the basement, conception and execution of the strategy were consulted, among them: Cochrane Systematic Reviews, Operational Manual, Evidence Guide, Guidelines, ENFAC Bulletin and others national and international studies. Subsequently, the coverage of the politic itself was evaluated through the quantitative analysis of data available in semi-annual reports by the Department of Basic Care (DAB) of the Ministry of Health's (MS). The results from the evaluation carried out in 2017 showed that less than half of the politic goal $(30 \%), 20 \%$ in the Southeast region, $34 \%$ in the State of São Paulo, where achieved. There are some municipalities, such as Limeira, SP, which, even though adhering to NutriSUS, did not implement the strategy. It is known that the strategy is effective in relation to the nutritional status of the supplemented children. Studies pointed out that there are positive results in reducing the prevalence of micronutrient deficits and anaemia, for example. In conclusion, there are a lack of studies related to the strategy implementation management, and it is important to seek answers on why the Nutrisus was elaborated with a considerable distance from the implementation practice, in order to establish relationships between efficiency and effectiveness of public politics.

\section{PHARMACEUTICAL CARE FOR THE PATIENT IN FAMILY HEALTH} STRATEGY.

Jose Vitor Tolloto Fernandes, Rubens Bedrikow.

The research propound for studying the pharmaceutical care of the patient in the Family Health Strategy, therefore, the clinical practice of pharmaceutical. It have been know how those professionals have experienced this practice so wispy known until recently, inside the conception that pharmaceutical care are no longer limited on the treatment, and started to care more about the emphaizes people caring. It was a qualitative research that used semistructured interviews with pharmacists who work in family health unities. Researchers employ the thematic analysing techniques to explore the collected material.

40.

IMPORTANCE OF INTEGRATIVE PRACTICES IN PRIMARY CARE WITHIN THE UNIFIED HEALTH SYSTEM.

Lourene Silva Santos, Gabrielly Silva Santos

The insertion of Integrative and Complementary Practices into public health systems has been much discussed nationally and internationally. In Brazil, the National Policy on Integrative and Complementary Practices guides the inclusion of practices such as acupuncture, homeopathy, auriculotherapy and ozonotherapy in the Unified Health System. Integrative and complementary health therapies have gradually become a reality in the primary health care network all over the country. This advance can be understood as an expression of a movement that identifies itself with new ways of learning and practicing health, since integrative practices are characterized by interdisciplinarity and unique languages, which are in opposition to the highly technological vision of health that prevails in today's society, dominated by health covenants whose main objective is to generate profit and to fragment the treatment of the patient in specialties that do not account for the totality of the human being in search of remedy for their ills. This article analyzes the advantages of implementing integrative practices in SUS, exposing and explaining the operation and potential of acupuncture in particular, and to report its use in the prevention, reception and treatment of some diseases. The results showed positive effects of this branch of Traditional Chinese Medicine in the prevention and treatment of certain pathologies, but further studies are still needed to confirm its efficacy. These integrative practices, coupled with traditional medicine, strengthen SUS principles, especially integrality and prioritize humanized and individualized attention.

\section{ESOPHAGEAL CANCER: EPIDEMIOLOGICAL STUDY AND FOLLOW UP OF} SURGICAL PATIENTS.

Pedro Pazini de Souza Chagas, Ana Beatriz Nascimento Costa, Rosângela Lucinda Rocha Monteiro.

INTRODUCTION: Esophageal cancer is relatively uncommon but has mortality rate very close to the incidence rate. In addition, it has been constantly related to the patient's life habits. When tracing an epidemiological profile of the patients affected with the disease, it is sought to evidence factors and risk groups located in the South of Minas Gerais and to test the items exposed in the literature. METHODS: This was a descriptive, observational and cross-sectional study, carried out with 50 patients, through interviews and analysis of medical records, with a qualitative and intentional approach to the data, which were collected at the Samuel Libânio Hospital, located in Pouso Alegre and at the Bom Pastor Hospital, locate in the city of Varginha, both of them in the State of Minas Gerais. RESULTS: The epidemiological profile obtained was in the majority of males ( $85.5 \%), 61$ years old, white, with and income $<1.5 \mathrm{MS}$, incomplete elementary school and farmers $(41 \%)$. Before the Cancer, $67.6 \%$ realized weekly physical activity, $65 \%$ used to consume coffee, $72.7 \%$ ingested alcoholic beverages, $83.6 \%$ smoked on average 1.3 packs / day, $45.5 \%$ liked warm drinks. $56.4 \%$ of the patients had cases of neoplasms in the family, $27.3 \%$ of them with a high TGI. CONCLUSION: As in the literature, the incidence of esophageal cancer was higher in males, $>51$ years, that consumes caffeine, hot drinks, alcohol and are smokers. However, the nitrous compounds present in the preserved foods had no expression in the cancer incidence, being interpreted as a less impacting factor in the studied region. Regarding green vegetables, the results were in agreement with what is evidenced in the literature, not presenting itself as a real protection factor. The majority of patients persisted with the use of the risk factors after being diagnosed and initiation of treatment, showing the inefficacy of the awareness and prevention means that already exist among patients undergoing treatment.

PREVALENCE OF SHOULDER PAIN IN HEMIPARETIC PATIENTS AFTER STROKE.

Débora Caroline Lourenço Monteiro, Carolina Telles dos Santos, Beatriz Horschutz Tocachelo, Sandra Regina Alouche, Heloise Cazangi Borges.

INTRODUCTION: Stroke is considered a first cause of disability in the world The clinical condition may consist of several disorders and hemiparesis is the most common symptom. It is common to present complications such as subluxation and pain in the hemiplegic shoulder, which may affect one third of the patients. The hemiplegic limb's motor impairment causes immobility and disuse, resulting in musculoskeletal structural reactions that occur mainly around the glenohumeral joint, affecting not only their daily function but also their performance during the physiotherapeutic treatment, resulting in a negative prognosis at the end of motor functional rehabilitation. OBJECTIVE: to identify the prevalence of hemiplegic shoulder pain post-stroke in individuals under treatment in a school-based clinic. It is expected that there is a high prevalence of pain in the individuals evaluated, as found in the literature. METHOD: A cross-sectional study performed at the Clinical School of Physiotherapy CEUNSP - Itu / SP, with individuals with sequelae of ischemic or hemorrhagic stroke. After approval of the work by the CEP / UNICID, participants were selected from the preestablished inclusion and exclusion criteria to perform the following evaluation procedures: Demographic, Mini Mental, Fugl-Meyer Scale and Visual Numerical Scale for pain (VNS). The obtained data were grouped in tables in Excel software and analyzed in frequencies, mean and standard deviations. RESULTS: The study included 29 patients, mean age of 62 years, with a higher frequency in women $(n=16)$, predominant ischemic stroke with $82.2 \%$, right hemisphere more affected with $62.1 \%$. Through the analysis of NVS, there was a prevalence of $58.62 \%$ of pain, observed greater number in the report of pain to the passive movement, agreeing with the literature where bedridden patients who need transfers refer pain. CONCLUSION: The prevalence of painful shoulder hemiplegic post stroke in 
the clinical school CEUNSP is $58.62 \%$. Among patients who had pain, their manifestation prevailed to the passive movement.

\section{ENCARCERATED WOMAN PERCEPTION OVER THEIR PRISIONAL ENVIRONMENT NEEDS.}

Vanessa Cristina Fanger, Celene Aparecida Ferrari Audi.

INTRODUCTION: Prisions are a great governamental opportunity to resocialize people privated from freedom, people that live on the verge of society and give opportunity of social ascension avoiding relapse. OBJECTIVES: Analise the answers of the encarcerated women in the Female Penitenciary of Campinas-SP, "If you could do something, what would be the first thing that you would do, here, so the life of women could be better?" METHODOLOGY: To apply a qualitative and quantitative analysis in relation to these women speech, obtained from 1013 woman under reeducation. Initially a quantitative analysis will be realized and them an analysis over the content of the women under reeducation speeches that were taken during field research. These analysis will be organized in a way that we will be able to analyse what is the priority for these wonem inside the subject. RESULTS $27 \%$ of the women under reeducation referred that the penitenciary should offer work, $14,5 \%$ would like to have health care attention at the prisional environment, $11,9 \%$ said that food should be better. Between other necessities, education was highlighted. CONCLUSION: Pre described public policies need to be implemented, so this population can live with all thei rights preserved.

\section{OCCUPATIONAL EXPOSURE TO BIOLOGICAL MATERIALS: ETHNOGRAPHIC} STUDY.

Izabela Barbieri De Freitas, Gabriela Chielli, Walter Manso Figueiredo, Marisa Marques Monteiro, Josiene Perpetua Dos Santos.

INTRODUCTION: Occupational exposure to biological materials has as a characteristic the contact of people involved in health services with potentially contaminated fluids. Those accidents are a challenge for professionals who work in the Hospital Infection Control and in the occupational Health Area, since it is a concrete fact the occupational acquisition of hepatitis $B$ and $C$ viruses and HIV by health workers. MATERIAL AND METHOD: This is a descriptive and quantitative research, based on information collected in the database of the Special Health Service of Araraquara, a school health unit of the School of Public Health of the University of São Paulo, part of the municipal health network, from January 2010 to December 2017. The following aspects were analyzed: profession, age, type of injury, type of biological material involved, use of personal protection equipment, vaccination, incidence curve of this type of injury over the years studied. RESULTS: In the period, there were 1335 cases of occupational accidents involving biological materials, with an average of 166.87 cases per year. Most of the workers were from the nursing area, students, physicians and dentists surgeons. The most prevalent lesion was percutaneous and the biological material present in most accidents was blood. It was observed that the use of personal protection equipment is not always done. Regarding the vaccination situation of the victims, there were still professionals without vaccination. There was also a significant dropout rate of the treatment. CONCLUSION: Biosafety is fundamental for professionals and students involved in health care services. Thus, professional qualification on accident prevention and safe behaviors exerts a positive interference in the context of occupational accidents with biological materials.

45. EPIDEMIOLOGICAL PROFILE OF SIDA IN THE MUNICIPALITY OF ARARAQUARA: ETHNOGRAPHIC STUDY.

Izabela Barbieri De Freitas, José Ricardo Fraçon Viana Alves, Julia Monteiro Giannini Romero, Henrique Olivieri de Lacerda, Walte Manso Figueiredo.

INTRODUCTION: The human immunodeficiency virus infection courses with a broad spectrum of clinical presentations, from the acute phase to the advanced stage of the disease in which opportunistic infections characterize Acquired Immunodeficiency Syndrome (SIDA). Despite the prevention campaigns and the institution of antiretroviral therapy, SIDA is still highly relevant today. MATERIAL AND METHOD: This is a descriptive and quantitative research. The present study aims to analyze the epidemiological variables in SIDA cases, in the city of Araraquara - SP, based on data from the Special Health Service of Araraquara, a school health unit of the School of Public
Health from University of São Paulo, part of the municipal health network. The analysis comprises the period from January 1995 to December 2015 and observes possible changes in their epidemiological profile over the years, associating with changes in the social factors involved in determining the disease. RESULTS: There is a change in the epidemiological profile of SIDA in the municipality: in 1995, SIDA was considered a disease of young adults and, over the years, it has been observed that it is moving towards age groups over 40. Regarding the hierarchical risk, SIDA is currently more prevalent among heterosexuals. It should be emphasized that the epidemiological profile in the municipality of Araraquara differs from the Brazilian profile in several variables. CONCLUSION: Given the results, it is recommended that, when reporting a SIDA case, anamnesis and the recording of important data to be analyzed should be made. In addition, the importance of early diagnosis of HIV infection, treatment with antiretrovirals and the realization of awareness campaigns for HIV virus prevention in the population stands out. So, more conscious individuals exert a positive interference in the context of SIDA.

\section{EMOTIONAL EXPECTATIONS OF A NURSING PERSONNEL: WHAT WILL REPLACE THE WORK? A QUALITATIVE STUDY WITH PROFESSIONALS WITH SCHEDULED RETIREMENT FROM A UNIVERSITY HOSPITAL.}

H. O. Santos, E. R. Turato

INTRODUCTION: Brazil walks to a country strongly of the elderly; in 2030 (IBCE), the number of over 60 years will be higher than that of individuals with up to 14 years of age. In this macrocontext, interested us personal experience of the transition to retirement for hospital health professionals university, whose care they helpedto organize, since this institution has completed three decades. This study has investigative aspects origin, as well as carries psychosocial relevance. Our theoretical premise is that the temporal approximation this 'turning point' is accompanied by intense emotional conflicts, many not aware. OBJECTIVE: Explore emotional meanings brought by professionals that go through the emotional process of the retirement, after long employment-and affective bond- with the Clinic Hospital of Unicamp. METHOD: Sample construction intentional, sequential by information in the institution. Subjects with the following inclusion criteria: a) Long employment at the Hospital de Clínicas da Unicamp (more than two decades); b) scheduled retirement. Instruments of data collection: semi-structured interview open-ended questions, notes from the observation / self-observation in the interview. Treatment of the data by Qualitative Content Analysis. RESULTS: The group studied, who accepted the invitation to participate, was constituted by 06 (six) interviewees of the $\mathrm{HC}$ nursing staff, who saturated thethe following topics: (1) Fantasies about what will symbolically place of work (2) Date marked while 'an elephant taken from on top of me (3) The irreplaceability at work: perception as a myth or a truth (4) Expectations of how the retirement as a rite of passage. CONCLUSION: The symbolic of retirement after long bond with health institution organizes the 'turn' in aspects beyond the objective organization of new biographical phase.

47. EVALUATION OF THE EFFICACY OF DIFFERENT APPROACHES FOR THE DIFFUSION OF KNOWLEDGE ON NUTRITION AND EATING BEHAVIOR FOR UNDERGRADUATE AND GRADUATE STUDENTS FROM THE UNIVERSITY OF CAMPINAS, BRAZIL.

Ana Paula da Silva Rios, Karla Nogueira, Natália Ferreira Mendes, Lício Augusto Velloso.

Admission to university usually results in significant changes in eating behavior. Dietary changes, coupled with the stress of the academic environment and lack of time for physical activity, are some of the many variables that contribute to the increase in the incidence of overweight and obesity in graduate and undergraduate students. The aims of this study were: (1) to collect data about the eating habits of the students from the University of Campinas in Brazil and, based on this data, (2) to apply some strategies of diffusion of knowledge that could promote not only the awareness on these subjects but also stimulate changes of eating habits. For that, in the first stage of this study, several data were collected throughout a questionnaire application with questions about nutrition and eating habits. In the second stage, three strategies of diffusion of knowledge on these themes were used. In the third phase of the study, a new application of questionnaires was carried out to evaluate the efficacy of the approaches chosen. The results of the first phase indicated that around $25 \%$ of the students interviewed were overweight or obese; $29 \%$ reported not practicing any physical activity, and $91 \%$ has never heard about the "Guia 
Alimentar para a Populacao Brasileira". The data collected after the nutritional education activities indicated that there were no significant changes in the acquisition of knowledge about these topics for the subjects of the study. In other words, the knowledge dissemination approaches used in this study were not effective in increasing knowledge about eating habits and nutrition or even stimulating changes in any life habits, which may be related to the short period of time in which they were carried out, as well as the lack of interest on the part of the students in memorizing new information or in adopting new lifestyles. This study supports the need to create new approaches for the dissemination of specific knowledge for this target audience.

\section{HEPATIC PROFILE EVALUATION IN PATIENTS AT A PRIVATE LABORATORY IN JACUTINGA-MG}

Larissa Regina Pereira, Marliely Crochiquia Silviéri, Thaís Louise Soares Patto.

The liver itself has metabolic functions, such as synthetic activity of compounds such as proteins, carbohydrates and lipids, detoxification and medicines metabolism, it owns excretory and secretory function, storage, protective functions, circulatory and blood coagulation. The aim of this study was to analyze the hepatic profile in the population assisted by a private laboratory in the city of Jacutinga, Estate of Minas Gerais, Brazil. A research with liver tests was conducted, a crosssectional study, with field research. The sample consisted of individuals who underwent examinations in a private laboratory in Jacutinga, during the months of January to December of 2017. Patient data were collected by consulting the database without identifying them, and a survey of all liver profile analyzes was performed during this period. The results of the serum glutamicoxaloacetic transaminase - SCOT or AST (aspartate aminotransferase), SCPT (serum glutamic pyruvic transaminase) or ALAT (alanine aminotransferase), gamma-glutamyl transferase (gamma GT), alkaline phosphatase and bilirubin were collected to analyze the hepatic profile. Were collected from 887 exams, of which 479 were female and 408 were male, the age group which the most exams were taken, was in those over 60 years old with 262 exams performed. The SCOT and the SCPT exams were the most requested among all the exams, obtaining $24.44 \%$ and $15.68 \%$ of the altered exams respectively. It can be concluded in this paper that the most requested tests for evaluation are the SCOT and the SCPT. A greater amount of altered results was found in the male sex in relation to the female one. The months in which exams were performed varied with the number of altered exams, and most of the altered exams of SCOT and SCPT was found in November and March, October and August for the Cama-gluamil Transferase, Alkaline Phosphatase and Bilirubin exams, respectively.

\section{RELATIONSHIP OF ESTHETIC PROCEDURES AND THE AGE OF WOMEN IN} JACUTINGA-MG.

Marliely Crochiquia Silviéri, Larissa Regina Pereira, Gleidson Juliacci Patto, Thaís Louise Soares Patto.

Life expectancy and the search for eternal youth have been increasing in recent years. Increasingly, people are looking for miraculous esthetic forms and methods that make physiological aging slow down in their lives, boosting their self-esteem. The aim of this study was to verify how esthetics is present in women's lives and at what age this procedure is most sought after. This research was conducted through a questionnaire interviewing women between the ages of 18 and 70 years old in the city of Jacutinga, State of Minas Cerais, Brazil. The variables of the study were defined according to: schooling, age, marital status and family income. When analyzing the results of the participants' profile, it can be observed that the age group with the highest percentage was between 21 and 30 years old, totaling $37 \%$ of the interviewees. Single women prevailed in the survey, reaching $52 \%$, there was a predominance of $71 \%$ from family income from 1 to 3 salaries, regarding to schooling, $55 \%$ of the interviewees have completed high school. Of the interviewees, $62 \%$ performed some type of esthetic procedure. Regarding to age and the procedures performed, peeling was performed between 31 to 40 years old ( 14 women), respectively, and also in the age range of 31 and 40 years old, 10 women performed laser hair removal and 8 women, lymphatic drainage. It was observed that women between 31 and 40 years old are the ones who make the most esthetic procedures. Nevertheless, the statistical tests did not indicate differences between the means of the ages and also the marital status, but presented a statistical difference between the participants' income. Thus, it can be concluded that income was a determining factor for women to perform the procedures.

50. ASSESSMENT OF THE EFFECTIVENESS OF THE THEORETICAL-SCIENTIFIC KNOWLEDGE DISSEMINATION ABOUT NUTRITION AND HEALTHY DIET FOR STUDENTS IN THE HEALTH AREA, RESIDENT PHYSICIANS, AND HEALTH PROFESSIONALS OF THE UNIVERSITY OF CAMPINAS IN BRAZIL.

Karla Morais Nogueira, Ana Paula da Silva Rios, Milena Monfor-Pires, Natália Ferreira Mendes, Lício Augusto Velloso.

Promoting, repairing and caring for the people health is one of the greatest aspirations of health professionals. However, contra-intuitively, the lifestyle of most of these does not fit with the recommendations and guidelines they make to their patients. As a consequence of this behavior, we have been observing an increased overweight and obesity incidence in the last years, not only in health professionals but also in students and residents in this area. Faced with this reality, in the first stage of this study we have collected several data on the life habits of students, residents and health professionals of the University of Campinas and applied a questionnaire with questions about nutrition and healthy diet. From the information obtained, two strategies of diffusion of knowledge on these themes have been used: (1) video-animation and (2) flyers. In the last phase of the project, which is still ongoing, we will be applying a new questionnaire for evaluating whether the chosen methods of knowledge dissemination have been effective in influencing people positively with respect to the adoption of healthy life habits or, at least, contributed to the acquisition of new knowledge about healthy eating habits.

\section{PROFILE OF QTC INTERVALS IN HEALTHY VOLUNTEERS FROM SÃO PAULO STATE, BRAZIL.}

Renan Donomae Iwamotob; Ronilson Agnaldo Morenoa; Fernanda Maria de Oliveira Nigro Fernandesb; Raul Cléverson Doloresa; Gilberto de Nuccia.

INTRODUCTION: QT prolongation is related to the development of ventricula arrhythmias and risk of sudden death. Studies are controversial when discussing about tolerable limit of variation for QT interval in order to prevent cardiac complications. There are few studies on electrocardiographic findings in populations in Latin America and Brazil. Thus, the present study aimed to collect data such as age, Body Mass Index (BMI), Heart Rate (HR), QT interval from ECG of a population from the metropolitan region of Campinas city, São Paulo, and compare obtained results with other studies in different populations. METHODOLOGY: Retrospective analysis of data from 500 healthy volunteers, 300 women and 200 men, primarily recruited for a bioequivalence study. RESULTS: No statistical significance were observed when comparing mean age and BMI: $34 \pm 12.2$ (age) and $25 \pm 2.7$ (BMI) for women; $32 \pm 9.7$ (age) and $25 \pm 2.5$ (BMI) for men. The mean QTC and HR were statistically higher for men ( 410 \pm 30.3 and $72 \pm 10$, respectively) compared to data obtained for women ( 389 \pm 29.9 and $66 \pm 10.3$, respectively). No statistical differences were observed analyzing data subdivisions according to the self-declared race. CONCLUSION: Our results suggest that self-declared race had no significant weight on analyzed data and QTC interval findings showed similarities with scientific literature. Differences were relative to the amplitude of results that can be explained by mean age and BMI found in studied population.

52. TRIPLE EPIDEMY: A COMPARISON BETWEEN THE PREVALENCE OF ARBOVIRUSES IN THE STATE OF MATO GROSSO DO SUL IN RELATION TO THE BRAZILIAN NATIONAL AVERAGE BETWEEN 2015 AND 2017. Viviane Batista Santos, Raquel Areias de Oliveira

Arboviruses are viral diseases transmitted by arthropods that are globally distributed, and may have an endemic and often epidemic picture in favorable climatic regions, such as Brazil. In the Brazilian territory, a high prevalence of three diseases caused by arboviruses transmitted by the Aedes aegypti mosquito: dengue, Zika and chikungunya. In the state of Mato Grosso do Sul, the prevalence rate of these arboviruses follows the trend seen throughout Brazil between the years 2015 and 2017, reaching, and in some cases, exceeding the national average, and alerting the need for better epidemiological studies, in order to improve and qualify the national and state action plan. This plan of action is divided into three fronts: to inform, educate and mobilize, based on epidemiological surveillance; to control the vector and organize the health care and 
education to the population. The sum of knowledge about these diseases so prevalent in the state and national scenario, and the comparison made from epidemiological bulletins published by Datasus between 2015 and 2017, led to the conclusión that even though Mato Grosso do Sul did not integrate the group of the most populous states in Brazil, left a conjuncture in 2015/2016 in which it was similar or even exceeded in the number of cases of dengue national media, for a more controlled number of arboviruses cases and positively lower than the national average found in 2017.

\section{DOMICILIARY, TRANSITION OF CARE AND POST-DISCHARGE MEDICATION} RECONCILIATION: ROLE AND IMPORTANCE OF CLINICAL PHARMACIST.

Fernanda Maria de Oliveira Nigro Fernandes; Renan Donomae Iwamoto; Regina Célia Turolla de Souza.

The Clinical Pharmacist play a role in contributing to the reduction of medication errors and optimizing the clinical care provided to the patient. Additionally, contributes to the reduction of treatment costs. The attributions given to the clinical pharmacist in a hospital are extensive, but the main objective is to ensure that pharmacotherapy is effective, adequate and safe, promoting the rational use of medicines. The present work sought to discuss the role and importance of the clinical pharmacist inserted in a hospital institution, highlighting the professional's role as driver of the different stages of care for medication reconciliation. We performed a narrative review of the scientific literature indexed in international databases, including those findings considered important for theme discussion. We concluded that medication reconciliation is an important tool for improvement of patient care and should be a routine in a hospital in order to assure reduction of medication errors, improve pharmacotherapy adhesion and promote cost-cutting correlated to optimization of assist and rational use of medicines.

\section{LUMBAR FLEXIBILITY ASSESSMENT - LITERATURE REVIEW.}

Audrey Vendramini De Carvalho, Luiz Carlos Boaventura

INTRODUCTION: Assessment of flexibility is extremely relevant for the strengthening of evidence-based clinical practice. PURPOSE: Considering the high prevalence of problems related to lumbar segments, the present study analyzes the convergence of results of international scientific production on sagittal lumbar spine flexibility assessment found in online journals from January 2013 to June 2018. METHOD: Descriptive analysis of online peer reviewed articles, highlighting the following characteristics: 1) period; 2 ) context; 3) target population; 4) instrument; 5) study design; 6) thematics; 7) results; 8) countries involved; 9) journals. RESULTS: Thirty-two articles, distributed in 25 journals were analyzed. Analysis and tracking of movement (50.00\%) and Low back pain $(37.50 \%)$ featured as prevailing contexts. The most studied instruments were Inertial Sensors (28.12\%), Coniometer $(15.62 \%)$ and Inclinometer $(12.50 \%)$. The most significant themes were Reliability (64.06\%), Validity (17.18\%) and concurrent Validity (12.50\%). Sensors and Goniometer presented excellent or good reliability. The surveys validated Sensors, Schober OST and MST Test, universal Coniometer and twelve goniometer Applications. CONCLUSION: The presented scientific production expresses the need of standardization of current methods employed to assess lumbar spine flexibility, guided by criteria of reliability and validity of the instruments used for this purpose. Inertial sensors technology is in process of multiple developmental stages of its clinical potential and limitations. In this research prevail studies related to instruments based on direct angular references - goniometers and inclinometers, including smartphone applications. Scientific validation of the latest technologies available in smartphone applications aligns with reproducibility of recommended measurement, whether in clinical or academic context. Results corroborate the status of low back pain as a prominent theme in its relationship with reduced lumbar flexibility.

55. THE PROFILE OF ADMITTANCES TO HOSPITAL DUE TO NON-CONTAGIOUS CHRONIC DISEASES SENSITIVE TO PRIMARY HEALTH CARE IN RIBEIRÃO PRETO MUNICIPALITY.

Matheus Pupo Pereira Lima, Débora Cristina Modesto Barbosa.

INTRODUCTION: The Non-Contagious Chronic Diseases (NCCD) are an important public health issue worldwide. According to World Health Organization a significant part of morbidity and mortality due to NCCD can be avoided by Primary Health Care system. OBJECTIVE: To stablish the profile of admittances to hospital resulting from Non-Contagious Chronic Diseases Sensitive to Primary Health Care (NCCDSPHC) in the city of Ribeirão Preto, SP, Brazil, over the periods of 2008 to 2012 and 2013 to 2017. METHODS: It is an observational, descriptive, ecological study of time series, based on secondary data available by the Hospital Information System (SIH-SUS) and by the Brazilian Institute of Geography and Statistics (IBCE). The rates of hospitalizations for NCCDSPHC were calculated for 1,000 inhabitants, and the group of causes were studied by gender and age group, comparing both periods. RESULTS: Hospitalizations for NCCDSPHC showed rates of $8.34 / 1,000$ between 2008 and 2012 and 8.46/1,000 between 2013 and 2017. The main causes were heart failure, angina pectoris and cerebrovascular diseases. The evolution of the rates between both periods occurred differently by age group and gender. CONCLUSION: The study did not exhibit any remarkable differences in hospitalizations for NCCDSPHC rates between the periods. Regarding the group of causes, reduced hospitalizations rates for heart failure and high blood pressure were observed, as well as increased hospitalizations for asthma, pulmonary diseases, diabetes, angina pectoris and cerebrovascular diseases; these hospitalizations occurred differently by gender and age group. The results showed that a deep reflection regarding the determinants of hospitalizations for avoidable causes is needed.

\section{EVALUATION OF TUBERCULOSIS IN THE MUNICIPALITY OF PRESIDENTE PRUDENTE IN THE PERIOD FROM 2010 TO 2015.}

Gabriel Kazuo Ishibashi Tatibana, Amanda Valério de Almeida, William Cazarini Ito, Ingrid Lehmkuhl Rinaldi, Eraldo de Azevedo Coelho Junior.

INTRODUCTION: Tuberculosis is an infectious disease caused by Mycobacterium tuberculosis, known as Koch's bacillus. As a neglected tropical disease, Brazil suffered from an endemic disease, with 1.5 million deaths in 2014. Therefore, through the Directly Observed Treatment, ShortCourse (DOTS), End - TB / WHO, and the National Tuberculosis Control Program strategies, tuberculosis cases in Brazil exhibited a significant decline, and the Southeast region is where the highest incidence prevails until today. This pathology is divided into two periods: primary and the secondary, where the latter affects one-third of the world's population. The most frequent form of the disease is presented as Pulmonary Tuberculosis, and the most serious is the Miliar Tuberculosis, due to the systemic involvement whose predominance occurs in immunosuppressed individuals. OBJECTIVE: Evaluate the profile of tuberculosis regarding the criteria of sex, age group, HIV and AIDS in the municipality of Presidente Prudente from 2010 to 2015. METHODOLOGY: DATASUS portal was used to collect informed and notified data according to the following ratings: month of notification, confirmed cases by age group, confirmed cases by sex, confirmed cases according to the form of tuberculosis, confirmed cases by the type of entry and confirmed cases by HIV, confirmed cases by AIDS. RESULTS AND DISCUSSION: The year 2012 was the one in which the disease was prevalent, with 100 confirmed cases. It was also observed that the predominant age group and gender in the analyzed period are: Male, from 20 to 39 years old. It was found that there was a decline in the number of tuberculosis cases and new AIDS cases as from 2012. CONCLUSION: the obtained data in the municipality resemble those of the literature, and the former presents a reduction in its incidence levels. However, Tuberculosis is still one of the main responsible factors for death among HIV-positive patients.

\section{APPLICABILITY OF COMPUTERIZED TOMOGRAPHY AND MAGNETIC} RESONANCE IN ELDERLY WITH ACUTE RAQUIMEDULAR TRAUMATISM.

Pedro Nogarotto Cembraneli, Julia Brasileiro de Faria Cavalcante, Fabiana de Campos Comes, Dionei Freitas de Morais, João Simão de Melo Neto.

INTRODUCTION: Elderly present changes common to senescence and senis that favor the occurrence of spinal cord trauma (SCT). Radiological examinations contribute to a more accurate diagnosis, but there are few studies that analyze this specific population. OBJECTIVE: To verify the characteristics that guide the choice of the exam applied in the assessment, the therapeutic approach adopted and the prognosis in elderly patients admitted with acute SCT. METHODS: Retrospective study with data collection of medical records carried out in a tertiary hospital, approved by the Institutional Research Ethics Committee, n. 806,452. A total of 271 patients were analyzed, being analyzed in subjects with $<60$ and .60 years, to verify the applicability of computed tomography (CT) and magnetic resonance imaging (MRI), isolated and combined. RESULTS / DISCUSSION: Men's are 
more frequently submitted to MRI. The etiology of motor vehicle accident (. 60 years with a higher indication of $\mathrm{CT}$ ), fall of height (Elderly regardless of the exams) and trampling (elderly with a higher indication of $\mathrm{CT}$ ) were determinant in the choice of radiological examination. The presence of sensory and motor alterations was more frequent in the elderly who underwent the isolated examination. ASIA-A neurological status [ 60 years: isolated (CT); .60 years: combined] and ASIA-B/C/D ( $<60$ years: isolated) were important. Individuals with $<60$ years had more associated lesions, mainly in the upper limbs and in the thorax, being directed to MRI. Cervicalgia $(<60$ years: MRI), upper (.60 years: isolated) and low back pain (.60 years: MRI; <60 years: isolated) were symptomatologies determining the choice of examination. CT and MRI were more associated with the diagnosis of morphologies of the lesions involving bone and soft tissue, respectively. Conservative treatment was the main choice in the elderly. Elderly patients presented more complications and mortality when they underwent combined exams, probably due to the initial clinical picture. CONCLUSION: The results of the applicability of these tests contributes to a better prognosis for the patient and lower expenditures to the health system.

\section{USE OF LICIT AND ILLICIT DRUGS BY MEDICAL STUDENTS IN BRAZIL. Arthur Petta}

INTRODUCTION: The use of drugs is present and widespread throughout the population and there has been a certain increase in habit in some groups with emphasis on university students and specially medicine students. To know the main individuals exposed to this factor of risk for various diseases is essential to design therapeutic approach strategies. OBJECTIVE: To analyze drug use among medical graduates of several universities of Brazil according to publications from 2000 to 2018. METHODOLOGY: This is a integrative review of a bibliographic study, with quantitative and qualitative analysis, based on articles published between 2000 and 2018, in scientific databases such as Bireme, Lilacs, Scielo, and MEDLINE, using the following descriptors: Inhalant Abuse, Consumption of Tobacco-Derived Products, Alcohol Drinking in College, Street Drugs, Students, Medical. RESULTS: Six articles were selected, most published in journals in the area of psychiatry or medical education, between 2000 and 2010. In 10 years, from 2000 to 2010, alcohol consumption remained practically constant, but oscillated during this period. In the case of tobacco and inhalants, however, there was a decrease in those 10 years, but there were also oscillations in the consumption of these drugs during this period. CONCLUSION: Knowing the particularities of drug use among university students is of paramount importance for the early detection of this consumption, the structuring of strategies / programs to minimize this consumption and support the students.

\section{BURN AWARENESS CAMPAIGN - THE KNOWLEDGE'S ACCESSIBILITY TO} THE PULATION.

Ana Paula da Silva Rios, Gustavo Henrique de Brito Santos, Isaque Miguel Pires, Lucas Militão, Gustavo Pereira Fraga e Thiago Rodrigues Araujo Calderan.

Burn injuries are considered a national public health problem, not only for the treatment costs but also for the damages it causes to the patient's physical, psychological and social health. According to the Ministry of Health (Ministério da Saúde), more than 1,000,000 cases occur per year, with 2,500 of these cases resulting in death, as a direct or indirect result from the lesions.June was chosen as the awareness month due to the increase in the number of burn injury cases - up to $30 \%$ - due to the increased contact with heat sources during June festivities, according to data gathered from Burn Centers throughout the country. With that in mind, the Unicamp Trauma League, through the COBRALT/SBAIT's program "Salvando Vidas" (Saving Lives), together with students holders of BAS Scholarships (Social Aid Scholarship - Unicamp), created a timeless data propagation and prevention campaign, showing how to act in certain situations in order to avoid burn wounds and what to do in the occurrence of them. The used method for this was a draw my life style video and a folder, which spread the content in a succinct form. The available video (https://m.youtube.com/watch?v=WTTebmyujn4) has reached around 1.2 million viewers and 1,000 folders were delivered to the population. All the material was made available online and through the institutional email, reaching 40,380 people. The campaign range reached through partners' websites and Whatsapp sharing cannot be exactly determined, but it's estimated to have reached more than 42.5 thousand people. We know we cannot quantify the direct impact in the new cases prevention, but we delivered the information to the population and increased their awareness regarding a very important disease.

60. CHARACTERIZATION OF THE PROBLEMS INVOLVED IN THE CARE OF THE CHILD WITH TRACHEOSTOMY AND ITS REPERCUSSIONS ON THE QUALITY OF LIFE OF THE CAREGIVER.

Felipe Montevechi Luz, Débora Bressan Pazinatto, Nayara Soares Lacerda, Ana Márcia C. Mendes-Castillo, Rebecca K. Maunsell.

The pediatric patient submitted to tracheostomy requires complex care and intensive and multidisciplinary follow-up due to the imminent risk of airway obstruction. However, the reality is a health service with unprepared professionals, a shortage of basic materials, an absence of quick access to a specialized service and, above all, the constant overload, inability and concern of the caregiver, whose quality of life is impaired. The objective of the study is to describe the characteristics of children with tracheostomy and their caregivers and evaluate the repercussions on the quality of life of their caretakers. Observational prospective cohort study. Caregivers of children with tracheostomy accompanied at HC Unicamp were invited to participate in the study. They answered a questionnaire full of demographic questions concerning the child, his caregiver, his social conditions and needs related to the tracheostomized condition and the WHOQOL-Bref quality of life questionnaire. A descriptive and comparative analysis of the data was performed to identify the factors with the greatest impact on quality of life. Participants included 45 caregivers of tracheostomized children. The average age was 4.9 years. Tracheostomies were performed on average at the age of 1.9 years and the mean length of stay with tracheostomy in the group was 2.9 years. About 45 children, $53.3 \%$ depend on public transport to move around. More than $60 \%$ don't receive home care and $88.8 \%$ follow up with at least two specialties. Respiratory internation was frequent, $60 \%$ were hospitalized after having performed the tracheostomy at least once and $22.2 \%$ more than 4 times. The occurrence of hospitalizations and length of stay with tracheostomy were related to worse quality of life. The rational flow of access to inputs and multidisciplinary monitoring, which allows a smaller number of displacements and hospitalizations and decantation as early as possible could impact the quality of life of this population. Most children with tracheostomy are preschoolers, don't attend child daycare centers and are cared for by their mothers. It's huge the need to travel for the treatment, which demands resources and time. The quality of life of this population is affected by recurrent hospitalizations due to respiratory problems and length of stay with tracheostomy.

61

EVALUATION OF HEARING, LANGUAGE AND MOTOR DEVELOPMENT IN INFANTS WITH RISK INDICATORS FOR HEARING LOSS

Júlia Roja Tavoni, Maria Cecília Marconi Pinheiro Lima.

INTRODUCTION: Monitoring the development of infants with risk indicators for hearing loss is justified by the importance of early diagnosis of possible disorders in the global development. The main goal of this study was to evaluate auditory function and language development and the environmental conditions of infants with hearing loss risk indicators. METHODOLOGY: All the infants were born in good conditions, remained under rooming-in care after birth, had normal results in the Otoacoustic Emissions test and had one or more risk indicators for hearing loss. To evaluate the auditory function we performed the cochlear-palpebral reflex test and auditory behavioral evaluation. To evaluate language development and aspects related to orofacial motricity we used the protocols Early Language Milestone Scale and the Protocol for Oral Motricity Assessment. For the motor development analysis we used the instrument Affordances in the Home Environment for Motor Development - Infant Scale. The research was approval by the Research Ethics Committee of the School of Medical Sciences of the State University of Campinas (UNICAMP) under Protocol No. 808.835, Sep. 23rd, 2014. RESULTS: Twenty-seven infants were evaluated and the most common risk indicator in the sample were: family history of congenital hearing loss and use of ototoxic medication during gestation. The data and the analyses carried out were compatible with the literature that children with risk indicators for hearing loss are often inserted in environments that may contribute negatively to their overall development. CONCLUSION: Even in not favorable environment, infants showed a hearing and language development within the expected age range, confirming the reality that risk indicators should be interpreted as probabilities of harm. At the same time that the benefits for the infants were observed related to hearing and language monitoring, the non-adherence of the relatives is 
highlighted, as reflected by the non-attendance of the families to the outpatient follow-ups.

62. CLINICAL AND CYTOGENETICAL PROFILE OF CASES WITH GENITAL AMBIGUITY ACCORDING AGE AT EVALUATION AT A REFERENCE SERVICE OF DISORDERS OF SEX DEVELOPMENT.

Carlos Wustemberg Germano, Mayra de Souza El Beck, Gil GuerraJúnior.

RATIONALE: The delayed or erroneous diagnosis of a patient with a Disorders of Sex Development (DSD) may lead an incorrect definition of sex and connsequently serious psychosocial problems. There is no information in the literature on a large single-service case series reporting the frequency of DSD attended at different ages according to the etiology and reason for the consultation. Objective: To verify the clinical and cytogenetic profile of the patients with genital ambiguity according to the age of first evaluation. CASUISTIC AND METHODS: We collected data from age at the first visit, initial and final social sex, reason for the consultation, karyotype and etiological diagnosis of all cases attended our service exóloga service from June 1988 to December 2016. The case was included when the main reason of consultation was genital ambiguity and having a confirmed etiological diagnosis. RESULTS: In this period, 1,793 cases with suspected DSD were evaluated. Of these, 654 did not meet diagnostic criteria for DSD and 541 did not have genital ambiguity as main complaint genital ambiguity. 598 cases were Included in the study: $50 \%$ were evaluated before 1 year of age and $17 \%$ after 10 years of age; $130(22 \%)$ did not have sex defined at the first visit; there was a need to change sex in 17 cases from male to female, and 13 from female to male, and the final social sex was predominantly male $(67 \%)$. There was an evident predominance of cases with karyotype $46, X Y(68 \%)$, followed by $46, X X(22 \%)$ in relation to abnormalities of sex chromosomes (10\%). There was a predominance of cases of DSD with karyotype $46, X Y$ and presence of two testicles $(57 \%)$ in relation to DSD of gonadal origin (23\%) and DSD with karyotype $46, X X$ and presence of two ovaries (20\%). Up to 1 year of age, the cases of $X Y$ Partial $Y$ Conadal Dysgenesis, Mixed Conadal Dysgenesis, Congenital Adrenal Hyperplasia and Syndromic and Idiopathic Cases of DDS 46, XX and 46, XY predominated. After the age of 10 years, the cases of Klinefelter Syndrome, Testicular Regression, Hypogonadotrophic Hypogonadism, Complete Androgen Insensitivity and $5 \alpha$-reductase type 2 deficiencywere predominant. CONCLUSIONS: This study presented the largest number of patients with genital ambiguity treated in the same service showing the clinical and cytogenetic profile of these cases and presenting for the first time the frequency of etiological diagnosis by age group.

\section{QUALITY OF LIFE OF FAMILIES WHO HAVE CHILDREN WITH CDLS IN}

\section{BRAZIL.}

Aline Apis, Rafaela Catelan Martins Pereira, Marcela Cesaretti Borilli, Francisco Antonio Rojas Rojas, Lucimar Retto da Silva de Avó, Carla Maria Ramos Germano, Rui Fernando Pilotto, Débora Gusmão Melo.

Cornelia de Lange syndrome (CdLS) is a rare genetic disorder with an estimated incidence of 1: 50,000 individuals. The clinical picture of CdLS is varied, consisting mainly of facial dysmorphia, delayed pre and postnatal growth, defects of upper limb reduction, and intellectual deficiency (ID), usually severe to profound. This research aimed to investigate the quality of life of families (FQOL) who have children with CdLS in Brazil. This is a descriptive research that was developed with the support of the Brazilian Association CdLS (CdLS-Brazil). Data were collected using face-to-face forms with sociodemographic and clinical information, Barthel and Lawton a Brody's functional indexes, and the Beach Center Family Life Quality Scale (BCFQoLS). A total of 92 families participated in the study. Individuals with CdLS were 10.5 years on average ( \pm 9 years), $47(51.1 \%$ ) were male and only $12(13 \%)$ had a confirmatory molecular genetic testing. Clinically, 61 individuals $(66 \%)$ had gastroesophageal reflux, $32(35 \%)$ heart disease, 29 $(32 \%)$ epilepsy and $41(45 \%)$ limb alterations, characterized as mild in most cases (24/41). Regarding the schooling level, 19 people with CdLS (21\%) were literate. Regarding the basic activities of daily living (indoor) and the instrumental activities of daily living (outdoor), $27(29.5 \%)$ and $36(39 \%)$ of individuals with CdLS, respectively, were identified as totally dependent on supervision and $45(49 \%)$ were characterized as unskilled to handle money and $29(32 \%)$ as wheelchair users or with difficulty in locomotion. The results of the "Beach Center Family Life Quality Scale" showed an average score of $100( \pm 13.2$; maximum of 250$)$, being the domains "parenting" $(r=0.914 ; p<0.001)$ and "family interaction" $(r=0.804 ; p<0.001)$ the most strongly correlated with the total FQoL. Subjects with CdLS had an average score of 44.9 ( \pm 32 ; maximum of 100$)$ in Barthel's functional index, regarding the basic activities of daily living, and $1.7( \pm 1.5$, maximum of 8$)$ in the Lawton a Brody functional index, regarding the instrumental activities of daily living, indicating severe dependence. The correlation between Barthel's functional index and the FQoL was weak $(r=0.191)$ and not significant $(p=0.069)$. The correlation between Lawton a Brody's functional index and the $F Q$ oL was weak $(r=0.215)$, but significant $(p=0.040)$. Presence of epilepsy $(p=0.025)$ and difficulty in locomotion $(p=0.030)$, as well as family income less than $R \$ 3,000 /$ month ( $p<0.001$ ), need to receive social benefit $(p=0.004)$ and presence of young parents (mother $r=0.303, p=0.004$, father: $r=0.255, p=0.019$ ) were significantly associated with lower $F Q 0 L$ scores. On the other hand, access to supplementary health ( $p<0.001)$, access to molecular genetic testing $(p=0.024)$, higher paternal $(p=0.005)$ and maternal $(p=0.026)$ schooling, the fact that the mother worked outside home $(p=0.008)$, the parents lived together $(p<0.001)$, living in the same residence $(p<0.001)$ and professing some religion $(p=0.026)$ were associated with higher scores of FQoL. These results allow knowing the sociodemographic and clinical profile and some of the health needs of Brazilian individuals with CdLS, besides allowing analyzing how these aspects are able to influence the FQoL. Ultimately, it is intended that this study serves as a subsidy for the construction of a comprehensive care line for patients with rare genetic diseases in the Brazilian Unified National Health System.

\section{SUPPRESSION OF OTOACOUSTIC EMISSIONS IN SCHOOLS IN TYPICAL DEVELOPMENT: DIFFERENT EVALUATION PARAMETERS.}

Giovanna Cristina Bertelli de Lacerda, Thalita Ubiali, Maria Francisca Colella dos Santos.

INTRODUCTION: The efferent pathway has an inhibitory effect on the functioning of the outer hair cells, improving the ability to understand speech in noise, as well as being an innate protection mechanism against intense noise. The inhibitory effect of the efferent pathway can be investigated by suppressing $O A E$, but there is still a gap between the acquired scientific knowledge and the application of OAE suppression in clinical practice. This is due, in large part, to the different parameters used in several studies, making it difficult to reach consensus on the usefulness and viability of this tool as an evaluation in the differential diagnosis of auditory alterations. The most commonly used masking noise is white, at 6odB NPS intensity, however, this stimulus can trigger the acoustic reflex and thus interfere with the suppression response of the OAE. Therefore, we will also use 50dB NPS noise as another evaluation parameter. OBJECTIVE: To compare the magnitude of OAE suppression in the total response, with contralateral white noise under conditions 5odB NPS (Signal to Noise Ratio +10 ) and 6odB NPS (Signal to Noise Ratio 0). METHOD: To date, 30 normal hearing children aged 8 to 13 years have been evaluated. The TEOAE was collected with linear click stimuli at $60 \mathrm{~dB}$ SPL. Suppression research was performed with contralateral white noise at $50 \mathrm{~dB}$ NPS (Signal to Noise Ratio +10 ) and at $60 \mathrm{~dB}$ NPS (Signal to Noise Ratio 0). PARTIAL RESULTS: There was a statistically significant difference between the magnitude of the inhibitory effect obtained in the amplitude of the total TEOAE response, considering the noise of 50 and $60 \mathrm{~dB}$ SPL. The $60 \mathrm{~dB}$ NPS noise generated a greater amplitude of response. CONCLUSION: Based on the obtained results, it was verified that the $60 \mathrm{~dB}$ NPS noise generated a larger amplitude when considering TEOAE suppression.

\section{STUDY OF PROGRESSIVE OR LATE APPEARANCE HEARING LOSSES IN INFANTS WITH RISK INDICATORS.}

Daniele Stradioto Ortolan, Maria Francisca Colella dos Santos

INTRODUCTION: The first years of life are essential for auditory development. Considering that hearing loss does not always manifest itself at birth, it is important that infants with risk indicators be monitored. OBJECTIVE: To analyze the auditory development in the first year of life of the infants who passed the screening but had risk indicators for progressive or late hearing loss or changes in auditory processing. METHOD: Were evaluated infants who were hospitalized in the ICU of the CAISM/FCM/UNICAMP and pass in the auditory screening through the Automatic Brain Stem Auditory Evoked Potential but presents risk indicators. Group 1 consisted of 74 infants aged 6 to 9 months and group 2 was formed by 58 infants aged between 10 and 13 months. Behavioral observation was performed on verbal and non-verbal sound stimuli, visual reinforcement audiometry and immittanciometry. RESULTS: For sound localization, it was observed that in group $1,38 \%(n=28)$ of infants did not localize non-verbal sounds in the position indirectly 
downward and for group 2, 50\% $(n=29)$ of infants did not perform direct downward location. As for voice reaction, it was observed that in group 1, $15 \%$ of the children $(n=11)$ did not show a reaction when called by name and in group 2 , the occurrence was $5 \%(n=3)$. As for the auditory recognition of simple orders, evaluated in the infants of group $2,28 \%(n=16)$ showed a delay in the development of this ability, not responding to simple commands. No infants with progressive sensorineural hearing loss were found, however, it was found that $27 \%(n=9)$ of children in group 2 are with alteration of the middle ear. CONCLUSION: No evidence of progressive or late hearing loss in the first year of life of the evaluated infants was found, however, children with delayed development of auditory abilities were observed.

\section{ACUTE AND SUBACUTE PARACOCCIDIOIDOMYCOSIS: A PEDIATRIC} COHORT OF 131 PATIENTS.

Mariana Tresoldi das Neves Romaneli, Natália Rocha Tardelli, Ricardo Mendes Pereira, Antonia Teresinha Tresoldi.

BACKGROUND: The acute and subacute form of Paracoccidioidomycosis are systemic severe diseases, which affect children from endemic regions, such as the southeast region of Brazil, generally leading to lymph node enlargement, fever, malnutrition, anemia, eosinophilia, hypoalbuminemia and hypergammaglobulinemia. OBJECTIVE: To describe the characteristics of the largest existent cohort of pediatric paracoccidioidomycosis. Subjects and METHODS: Children and adolescents diagnosed with paracoccidioidomycosis, from 2 to 14 years were included in the study. The epidemiological, clinical and laboratorial data from those patients were obtained from their hospital records. A statistical analysis was performed in order to identify significant information. RESULTS: Within a period of 37 years, 151 cases of paracoccidioidomycosis were diagnosed and treated in 131 patients. The disease affected boys in preference to girls, specially the ones in the age range closer to puberty, and the most frequent laboratoria findings were anemia, eosinophilia, hipoalbuminemia and hypergammaglobulinemia. The complication rate was $29.8 \%$ and the mortality rate was $4.6 \%$. CONCLUSIONS: Simple clinical and laboratorial exams are able to strongly suggest the diagnosis of acute and subacute paracoccidioidomycosis, and authorize the beginning of the treatment even before the isolation of the fungus in biological samples, preventing the morbidity and mortality related to the disease.

\section{MILK INTAKE BY BRAZILIAN UNIVERSITARY STUDENTS.}

Maria Rayane Lima de Souza, Elizete Aparecida Lomazi, Lilian Helena Polak Massabki.

In the last decades, the world consumption of milk showed a drastic reduction. In Brazil, in the last five years, consumption per person decreased from 60 to 46 liters per year. One reason for this decline seems to be the perception that milk causes damage for health and that it can universally trigger gastrointestinal symptoms of lactose intolerance. However, the ability of adults with ontogenetic hypolactasia to overcome symptoms of lactose intolerance by consuming reasonable amounts of lactose has been extensively demonstrated in epidemiological and clinical studies and available scientific data do not justify the conception that milk ingestion would be harmful to health. Milk is an important nutritional source for adults, justifying an analysis of the current consumption trend and, if appropriate, considering food education measures. The objective of the present study was to investigate the average consumption of milk and milk products in college students. Descriptive, cross-sectional, observational, prospective study. Graduation students from the Faculty of Medical Sciences were invited to answer an online questionnaire on the consumption of milk and milk containing products. The questionnaire was sent to all undergraduate students by the Commission for Undergraduate Education. 312 individuals responded to the questionnaire applied, range age $16-48$ years, $57 \%$ female and $80.5 \%, 24$ years. $72 \%$ reported consumption of milk with lactose and about $50 \%$ consumption of $250 \mathrm{~mL}$ or more a day $11 \%$ only consumption of milk without lactose and $17 \%$ reported not ingesting milk. Among milk non-consumers, $32 \%$ ingested dairy products daily, $35 \%$ ingested sporadically (,1x a week) and $17 \%$ did not ingest nor derived. $70 \%$ referred daily bowel habit, $24 \% 1 \mathrm{x}$ each 2 days and $6 \%, 3 \times$ a week/ (68\% women). Regarding the faecal aspect, $50 \%$ of the individuals have considered normal appearance, of which $72 \%$ consumed milk with lactose $11 \%$ described their feces as Bristol 4 or 5 (faeces softened to diarrhea), and $46 \%$ of these individuals did not ingest milk or ingest lactose-free milk. $40 \%$ indicated faecal appearance compatible with constipation. CONCLUSIONS: Milk intake lower than dietary recommendations was observed in $30 \%$ of the investigated population. Women above the age of 24 represented the demographic profile with the owest percentage of consumption.

\section{PULMONARY TUBERCULOSIS EPIDEMIOLOGY ON CHILDREN OF THE CITY OF CAMPINAS (SP) - BRAZIL.}

Ricardo Cunha de Camargo, Rodrigo Otero Coelho, Maria de Fátima Campos Marciano.

Neglected Diseases (ND) are those which prevalence is consistent with social iniquity, therefore, Tuberculosis is understood as ND by the authorities. It is difficult to understand how Tuberculosis, a disease so well known, goes unnoticed by medical evaluations in the various health services on Brazil. This work reinforces, in a numerical way, how much the diagnosis of tuberculosis needs to be understood by health professionals. This study is based on the collection of data from the medical records of patients evaluations and/or notified in the service in which the study was made. The data are from the period between 2010 and 2017. Were collected information from 88 cases, but only 78 cases were selected, because 10 cases are derived from active search. In the medical records was searched information such as sex, district of the city in which it inhabits, gestational term at birth, immunization for BCG, other personal diseases, year of diagnosis, age at the diagnosis, age at the beginning of the symptoms, symptoms presented, the scoring criteria for diagnosis, presence of a chronic cougher, misdiagnoses, therapeutic scheme used, known adverse drug reactions, treatment complications, liver enzyme changes and number of days in the interruption of the treatment in case of drug induced Hepatitis. Among the results found, the total sample represents $37.3 \%$ of the number of notified cases in the city, with an average time between the beginning of symptoms and the diagnosis of 8,09 $\pm 7,98$ months. The most prevalent personal diseases were the atopy group (rhinitis, asthma and dermatitis) ( $41 \%)$. When diagnosed, patients presented, in general, clinical criteria $(92.31 \%)$, radiological criteria $(80.77 \%)$ and some known chronic cougher $(65.38 \%)$, the most prevalent were the family members. The main reported symptoms were cough $(84.62 \%)$, fever $(70.51 \%)$ and weight loss/stagnation (61.54\%). The main adverse drug reactions were nausea/vomiting/diarrhea with $64 \%$, followed by drug induced hepatitis with $32 \%$, with an average time of treatment interruption of $14,75 \pm 12.5$ days. The main interference on the treatment were upper airway infections $(71 \%)$, followed by problems on the treatment engagement $(45.2 \%)$. The study also revealed that only $20.51 \%$ of patients were diagnosed with Pulmonary Tuberculosis in the early first evaluation, with an increase in diagnosis in the second or third evaluation. Our study noted that there is a significant delay between the beginning of the symptoms and the diagnosis, opposing to the recommendations by the Brazilian national plan against Tuberculosis. The clinical manifestations of the disease are in accordance with the literature, but the adverse drug reactions found are not the most recurring. We demonstrate with this study that the public health service, despite being the holder of the treatment of Tuberculosis in Brazil, is not adequately able to make the early diagnosis of this disease.

\section{EFFECTIVENESS OF A SELF-REPORT QUESTIONNAIRE AN CENTRAL AUDITORY PROCESSING SCREENING TOOL.}

Tamíris Aparecida Novaes de Oliveira, Maria Isabel Ramos do Amaral.

INTRODUCTION: The development of oral language occurs satisfactorily unde the condition of normal hearing, considering the integrity of both peripheral and central auditory system. Central auditory processing (CAP) refers to the efficiency and effectiveness by which the central nervous system (CNS) utilizes auditory information, and includes several mechanisms that underlie specific abilities that allow the analysis and understanding of auditory information. Early detection and intervention of auditory disorders are important and current guidelines in the area have recommended the use of auditory questionnaires and/or checklists as a complementary screening tool in a school environment. OBJECTIVE: to analyze and discuss the use of the self-perception questionnaire as a screening tool for hearing skills applied in children with and without school difficulties. METHOD: a cross-sectional, descriptive study conducted in partnership with a public school and the University of Campinas (UNICAMP) Audiology Laboratory, after ethics approval (CEP/UNICAMP 1.538,278). 52 children (27 girls; mean age of 9.13 years \pm 0.57 ), participated of a screening program at schoo (phase 1). Screening procedures included: meatoscopy, auditory tasks and 
a self-report questionnaire, adapted from the validated tool "Scale of Auditory Behaviors - SAB". The affirmative sentences were transformed into direct questions and an "example situation" was added before the question in order to help the child. The children answered 12 questions regarding how often a given situation occurs. Total score ranges from 12 to 60 points. The result was compared to the original classification, which suggests a score lower than 45 points as a risk for auditory processing disorder (CAPD). In phase 2,38 children with a mean age of 9.13 years $( \pm 0.57)$, being 26 girls ( $68.4 \%$ of all), attended the Audiology Laboratory for diagnostic evaluation. Children underwent a basic audiological evaluation and a CAPCentral auditory processing disorder (CAPD) behavioral assessment. The diagnosis was based on at least two altered tests of the behavioral battery. For further analysis, children were divided into Group I (without school difficulties) and Group II (with school difficulties) based on the evaluation of the teacher responsible. RESULTS: groups were considered homogeneous regarding age $(p=0.346)$ and sex $(p=0.695)$. Gl presented a mean score of $45.0( \pm 8.06)$, which was a significant better performance compared to GII $(p=0.022)$. Regarding to diagnosis of CAPD, seven children from $\mathrm{Cl}(25 \%)$ and six from Gll $(60 \%)$ were classified with CAPD. There was no statistical correlation between the performance on the questionnaire and the diagnostic evaluation, except for the Dichotic Test of Digits in the left ear $(p=0.010)$. CONCLUSION: The self-report questionnaire was considered an adequate tool to distinguish children from $\mathrm{Gl}$ and Cll. The use of this tool must be combined with other screening procedures in order to contributes to early and assertive referral for central auditory processing evaluation.

70. CLINICAL-EPIDEMIOLOGICAL PROFILE OF INFANTS OF DIABETIC MOTHERS'S ADMITTED TO A NEONATAL INTENSIVE CARE UNIT AT THE HOSPITAL DAS CLÍNICAS SAMUEL LIBÂNIO.

Mayara Silva Volaco, Bruna de Miranda Maioni, Ana Beatriz Teodoro Borges, Anna Luiza Pires Vieira.

OBJECTIVE: To evaluate the clinical and epidemiological conditions of newborns infants of diabetic mothers (NBIDM) hospitalized in a neonatal intensive care unit. INTRODUCTION: Gestational diabetes mellitus (CDM) is the most prevalent metabolic problem present in pregnancy. The pancreatic function is insufficient to overcome insulin resistance due to placental secretion of diabetogenic hormones. Mother and baby are affected by DMC at the risk of developing undesirable outcomes, such as macrosomia, fetal distress, metabolic disorders, hyperbilirubinemia, growth imbalance, congenital anomalies, prematurity, perinatal asphyxia, respiratory distress and complications metabolic diseases. This study aims to report the perinatal outcome of diabetic mothers admitted to the neonatal intensive care unit. METHODS: This is a historical cohort, including all live newborns infants of diabetic mothers (NBIDM) hospitalized in a neonatal intensive care unit at Hospital das Clínicas Samuel Libânio in the first Quarter of 2017. RESULTS: 87 newborns were hospitalized in this period, the percentage of NBIDM was approximately $10 \%$, with mean gestational age of 35 weeks, weighing $3035 \mathrm{~g}$ and $55 \%$ were classified as large for gestational age. Preterm labor accounted for $55 \%$ of deliveries and a decompensation of maternal pathology by $22 \%$. Regarding maternal epidemiological data, they were on average 27 years old, $44 \%$ were primigravidae, $44 \%$ were diabetic type 1 and $22 \%$ were gestational diabetic. A $100 \%$ of deliveries were cesarean. During the clinical course, $77 \%$ of newborns had respiratory distress and required ventilatory support, $44 \%$ hypoglycemia, $33 \%$ congenital malformation, and 2 of the central nervous system and 1 of the cardiovascular system. Neonatal mortality was $11 \%$, and death was a consequence of cardiac malformation. CONCLUSION: The diagnosis of diabetes mellitus before or during a gestation is related to the worst maternal and fetal prognosis, especially with regard to the increase of rates of spontaneous abortion, congenital malformations, fetal death, macrosomia, prematurity and respiratory metabolic disorders of the neonate.

\section{EPIDEMIOLOGICAL AND CLINICAL PROFILE OF EXTREMELY PREMATURE} INFANTS ADMITTED IN A NEONATAL INTENSIVE CARE UNIT.

Bruna de Miranda Maioni, Ana Beatriz Teodoro, Anna Luiza Pires Vieira, Mayara Silva Volaco.

OBJECTIVE: To evaluate the clinical and epidemiological conditions of extreme preterm newborns (NB) in a neonatal intensive care unit (NICU). METHODS: This is a historical cohort, including all live births over $500 \mathrm{~g}$ and gestational age less than 30 weeks admitted to the Neonatal Intensive Care Unit of an University Hospital in the 1st Quarter of 2017. RESULTS: 87 NB were hospitalized in this period, the percentage of extreme prematurity was approximately $13 \%$, with a gestational age of 27 weeks, weighing $968 \mathrm{~g}$ Preterm labor was accounted for $70 \%$ of deliveries, related to the maternal epidemiological data, they were on average 26 years old, $50 \%$ were primigravidae, $75 \%$ were prenatal, $25 \%$ had Specific Hypertension Disease in pregnancy (SHD) during pregnancy. Regarding the peripartum, $50 \%$ received at least 1 cycle of corticoid and $75 \%$ were cesarean deliveries. The mean time of hospitalizationin the NICU was 48 days, during the clinical course, $58 \%$ had sepsis, $33 \%$ BPD, 25\% PDA and $25 \%$ had PIVH. Neonatal mortality was $25 \%$, whereas 2 newborns died in the first hours of life, they had a mean of 23 weeks, 530 g weight and their mothers did not perform prenatal care. CONCLUSION: Extreme prematurity is a major risk factor of death, with perinatal conditions determining this outcome. Thus, it is concluded that the broad knowledge of the population served in the neonatal intensive care units allows the planning of a more appropriate care and the attempt to reduce the mortality rates.

\section{ANALYSIS OF WHOLE EXOME SEQUENCING IN ADOLESCENTS WITH COMMON VARIABLE IMMUNODEFICIENCY (CVID) WITH AUTOIMMUNITY AND LYMPHOPROLIFERATION PHENOTYPE.}

Janine Oliveira de Souza, Lia Furlaneto Marega, Janine Schincariol Sabino, Marcus Vinícius da Costa Pedroni, Marcelo Ananias Teocchi, Maria Marluce dos Santos Vilela.

CVID is one of the major primary immunodeficiencies of antibodies characterized by reduction of two classes of immunoglobulins and five clinical phenotypes with heterogeneous morbidity and prognosis: autoimmunity, lymphoproliferation, malignancy, recurrent infections and inflammatory disease of the gastrointestinal tract. The whole exome sequencing (WES) is an effective method to identify the genotypic variation and, consequently, the prognosis of this disease. The objective of this study was to investigate patients with humoral deficiency of unknown origin, phenotype of lymphoproliferation and autoimmunity. WES was applied in samples from 12 patients with ALPSU, all with the mentioned phenotype and without pathogenic mutations in FAS and FASLC. The estimated coverage for each patient was 100x of the exome. The analysis by bioinformatics provided the construction of a database of variations for each patient. The candidate genes were then chosen based on specific literature on the pathophysiology of PIDs and on the analysis of apoptosis signaling pathways. Twelve patients from the Hospital de Clínicas of UNICAMP were studied with a genetic molecular diagnostic yield of $67 \%$. The onset of symptoms was between 3 months and 16 years and the diagnosis was between 4 and 28 years. $91.6 \%$ of the patients had thrombocytopenia, $33.3 \%$ neutropenia, $25 \%$ leukopenia, $41.6 \%$ hypogammaglobulinaemia, $41.6 \%$ hepatosplenomegaly, $41.6 \%$ splenomegaly, $58.3 \%$ lymphadenopathy and $25 \%$ anemia. WES makes possible to accurately diagnose individuals with atypical or complex manifestations. However, for most individuals with CVID, genetic defects have not been identified, which leads to an ongoing effort to find new genes for the CVID phenotype.

\section{EVALUATION OF ANTIOXIDANT PEPTIDES EFFECTS IN MICE SUBMITED TO EPILEPSY EXPERIMENTAL MODEL INDUCED BY PILOCARPINE.}

Angela Hyun Ji Kim, David Ramos da Silva.

INTRODUCTION: Epilepsy is a chronic disease, which affects approximately 65 million people worldwide, with most patients from developing countries. One of the changes caused by the disease is the elevation of reactive oxygen species (ROS) in the brain and, consequently, oxidative stress, causing damage to the neurons. Currently, despite the availability of 15-20 antiepileptic drugs, resistance to these drugs and the clinical problems they cause compromise more than a third of patients with epilepsy. Thus, in recent years, the number of studies aimed at the discovery of compounds that may be more efficient for the treatment of this pathology, which includes antioxidant peptides, has been increasing considerably. OBJECTIVE: The main purpose of this project is to evaluate the effect of antioxidant peptides on the intensity of epileptic seizures in mice submitted to the experimental model of pilocarpineinduced epilepsy. METHODS: The proposed peptides were synthesized using the FmoC / tBut strategy. Cleavage of the peptide from the resin and removal of the side chain protecting groups were carried out in a mixture containing high content of TFA (Reagent K). After the whole synthesis process, the peptides underwent a characterization process in HPLC and mass spectrometry, in which the purity of the peptides was verified. For the induction of the status epilepticus, the animals $(n=60)$ were pretreated with a subcutaneous injection of methyl scopolamine at a dose of $1 \mathrm{mg} / \mathrm{kg}$, used to minimize 
the peripheral cholinergic effects of pilocarpine. Thirty minutes after this pretreatment, pilocarpine was administered intraperitoneally (i.p) at a dose of $300 \mathrm{mg} / \mathrm{kg}$. After two hours of status epilepticus, the animals were treated with $10 \mathrm{mg} / \mathrm{kg}$ diazepam intraperitoneally to interrupt the behavioral manifestations of epilepticus status (ES) and, thus, to ensure the survival of rats. Soon after ES recovery, the animals were moved to the vivarium, which is equipped with a $24 \mathrm{~h} /$ day video camera monitoring system to assess the number of seizures presented during the 21 days of treatment with the peptides. It should be emphasized that the treatment was initiated only after the presentation of the first spontaneous crisis. RESULTS: The proposed peptides I (H- $\beta$-Ala-His-OH) and II (H- $\beta$-Ala-His-Cln$\mathrm{Cln}-\mathrm{G} \ln -\mathrm{C} \ln \mathrm{OH})$ were satisfactorily synthesized and purified. In the present study, we used the experimental model of epilepsy induced by pilocarpine at a dose of $300 \mathrm{mg} / \mathrm{kg}$ for behavioral analysis of the animals and observation of the frequency of recurrent seizures for 21 days. For this, we divided the animals into 3 groups: Pilo $(n=4)$, Pilo + Peptide I $(n=4)$, Pilo + Peptide II $(n=4)$; with the peptide dose of $3 \mathrm{mg} / \mathrm{kg}$. In the Pilo control group, mean seizures were 3.76 crises / day. In the Pilo + Peptide I group, the mean was 0.77 crises / day and in the Pilo + Peptide II group, the mean of attacks was 1.64 crises / day. The results showed that there was $80 \%$ reduction in the frequency of attacks in the Pilo + Peptide group compared to the Pilo group, whereas in the Pilo + Peptide II group the reduction was $56 \%$ in the frequency of attacks when compared to the group Pilo control. CONCLUSION: The proposed I ( $\mathrm{H}-\beta$-Ala-His-OH) and II (H- $\beta$-Ala-His-Cln-Gln$\mathrm{Cln}-\mathrm{Cln}-\mathrm{OH}$ ) peptides were efficient in reducing the number of epileptic seizures of animals submitted to the model of pilocarpine-induced epilepsy in the order of $80 \%$ and $56 \%$, respectively, in relation to the pilocarpine control group. These results were corroborated by analyzes of the histological sections that showed greater hippocampal atrophy in the control group in relation to the groups treated with the peptides. However, although the model of epilepsy induced by pilocarpine is the most consecrated in the literature, this model leads to a mortality rate of around $60 \%$ and because it is unpublished results we would need one $n$ of animals per larger group to perform a statistical test for the validation of the results found.

STUDY OF THE EFFECTS OF AGOMELATIN ON MODAFINIL-INDUCED STEREOTYPES BEHAVIOR IN RATS (EXPERIMENTAL MODEL OF SCHIZOPHRENIA).

Beatriz Palma Aprobato, Cristina de Zotti Nassis.

BACKGROUND: Agomelatine is an antidepressant drug that acts as an agonist at melatonin $\mathrm{MT}_{1}$ and $\mathrm{MT} 2$ receptors and as an antagonist at the serotonin $5 \mathrm{HT}_{2} \mathrm{C}$ receptor. Treatment with agomelatine is notable for its effects on circadian rhythms. Evidence has accumulated to support the effects of agomelatine in psychiatric disorders, such as schizophrenia. An amplification of the antipsychotic effects of clozapine, when associated with agomelatine, was demonstrated in a pilot clinical trial. As clozapine is the most useful atypical antipsychotic in refractory schizophrenia, and also what causes the highest rate of severe agranulocytosis, such amplification could allow the reduction of antipsychotic doses, thus reducing the incidence and / or severity of such an effect adverse effects, as well as increase the therapeutic efficacy of the drug in partial responders. However, there were no controlled experimental studies in the literature with the objective of studying this effect. This was, therefore, the objective of the present study. METHODS: The classic experimental protocol was used, based on the paradigm of stereotyped behavior induced by psychostimulants. 28 Wistar rats were divided into 4 groups $(n=7)$ and treated (oral) for 14 days, depending on the group, with saline, agomelatine $(1.0 \mathrm{mg} / \mathrm{kg})$, clozapine (7.0 mg / kg ) or with the combination agomelatine / clozapine (1.0/7.0 $\mathrm{mg} / \mathrm{kg})$. At the end of this period the rats received modafinil $(5.0 \mathrm{mg} / \mathrm{kg})$ ip. The mean values of stereotyped behavior scores recorded over 60 minutes (started 30 minutes after modafinil administration) from each treated group were compared with those from the control group and the mean clozapine group was compared to that from the association by the Mann- Whitney ( $p<0.05$ ) RESULTS: Based on the means and respective standard deviations of the sum of the stereotypy scores of each group during 60 minutes, it was analyzed that the groups receiving clozapine (22.7 \pm 2 ) and the association between clozapine and agomelatine $(11.4 \pm 2)$ had a significant reduction in stereotypy scores when compared to the control group $(39.5 \pm 3)$. the group receiving agomelatine $(38.7 \pm 2)$ did not show a significant difference in the score when compared to the control group. Comparison between the clozapine and clozapine / agomelatine groups also revealed that the score for the group receiving the combination was significantly lower than that for the antipsychotic alone. CONCLUSION: The combination of agomelatine and clozapine was able to significantly reduce the stereotyped behavior induced by modafinil when compared to clozapine alone. The agomelatine alone was not able to significantly reduce the stereotyped behavioral scores in the model used, but nevertheless significantly amplified the effect of clozapine in such model.

\section{TOPICAL APPLICATION OF GLUTAMIC ACID REGULATES PROLIFERATION} OF KERATINOCYTES AND PLASTICITY OF MACROPHAGALS IN THE SKIN. Beatriz de Andrade Berti, Carlos Poblete Jara, Eliana Pereira de Araujo.

Glutamic Acid is the main excitatory neurotransmitter in neurons. Detrimental effects, either increasing or decreasing their activity, result in the manifestation of neuropsychiatric disorders, such as schizophrenia and Alzheimer's disease. Interestingly, sharing the same embryonic origin, keratinocytes, also express glutamic acid receptors, as well as their transporters. Abnormal distributions of the glutamic acid receptor have been shown in hyper proliferative models such as psoriasis and skin regeneration. However, the biological function of glutamic acid in this tissue has not been described. Here we show for the first time that the topical application of Glutamic Acid, at different concentrations, modulates keratinocyte proliferation. Moreover, topical application of Glutamic Acid decrease expression of genes related to macrophage differentiation as well as activates genes related to apoptosis signaling in mice. These results suggest that Glutamic Acid and its receptors play a critical role in maintenance of skin integrity and immunomodulation. Our results could be the starting point to elucidate how the intake of industrialized foods enriched with Glutamic Acid modulate diseases in the skin.

\section{HMGA2 AND PLAG1 EXPRESSION IN PLEOMORPHIC ADENOMA TUMORIGENESIS AND ITS RECURRENCE AND IN THE PROGRESSION TO CARCINOMA EX PLEOMORPHIC ADENOMA.}

Louyse Vizotto Moura, Larissa Fernandes Rosa, Reydson Alcides de Lima Souza, João Figueira Scarini, Fernanda Mariano.

The pleomorphic adenoma is the most common neoplasm of salivary glands, they present benign course with slow growth, asymptomatic and discrete nodules that can become large masses if they are not treated. Recurrences of APs are common and increase the likelihood of malignancy occurring. Although benign, the AP may undergo malignant transformation to Carcinoma Ex Adenoma Pleomorphic, which although rare, is an aggressive tumor with frequent metastasis and death related to disease. CXAPs can be classified according to histological type (salivary duct carcinoma, myoepithelial carcinoma, myoepithelial carcinoma, epithelial carcinoma and undifferentiated adenocarcinoma) and according to tumor progression in relation to the capsule (minimally invasive and frankly invasive intracapsular carcinomas) The Pleomorphic adenoma gene 1 (PLAG1) and High mobility group AT-hook 2 (HMGA2) genes undergo rearrangements that have an association, previously reported in the literature, with the onset and progression of APs and CXAPS. HMGA2 is a gene that has the function of architectural transcription factor, modulating the three-dimensional conformation of the DNA and consequently modulating the expression of several genes. The PLAG1 gene is involved in cell proliferation through the control of various target genes. In normal tissues, its activity is high during embryonic and fetal development, but in adult life, however, its participation is low or absent, as is the case with the HMGA2 gene. In this study we analyzed the expression of PLAG1 and HMGA2 in 38 cases of PA, 36 cases of Recurrent AP and 41 cases of CXAP taking into consideration the histological subtype and degree of tumor progression. A significant association of PLAG1 with Pleomorphic Adenomas was found, while the HMGA2 gene had a relevant association with the malignant counterpart of the disease, CXAP. It was also observed a higher prevalence of HMGA2 expression in high grade and aggressive tumors, adenocarcinomas and salivary duct carcinomas. Our study showed that these genes are promising targets with regard to early diagnosis, more effective therapies and consequently lower morbidity from these neoplasms. More studies are needed to clearly understand the role of PLAG in the onset of Pleomorphic Adenomas and the relationship of HMGA2 with the process of malignancy and progression of the Carcinoma Ex Pleomorphic Adenoma.

77. EFFECTS OF Chlorella algae ON THE QUALITY OF LIFE OF PATIENTS WITH GLUCOSE INTOLERANCE AND TYPE-2 DIABETES. 
Caio Augusto Leme Fujiura, Mary Luci de Souza Queiroz, Cristiane Okuda Torello, Fernanda Martins, Tamara Cristina Lopes de Castro.

Nowadays, we have an ongoing Diabetes epidemic and the number of patients and deaths caused by complications from it tends to grow in the coming years. Pharmacological treatment of type-2 diabetes is effective and improves some metabolic parameters, but it has several side effects that, in many cases, can worsen the general health of the patient. Chlorella algae is a complete food and has nutrients such as carbohydrates, proteins, nucleic acids, amino acids, essential fatty acids [ômega-3 $(-3)$ e ômega-6($6)$ ], vitamins, dietary fibers, growth factors and antioxidants that promote health. It is an adaptogenic plant capable of modifying biological responses since it can increase the resistance of the host to a variety of chemical, physical and biological stresses without altering the normal functions of the organism. Recent studies from our laboratory have demonstrated that algae is able to prevent insulin resistance in obese animals by improving the signaling pathway of insulin in the liver, skeletal muscle and adipose tissue by increasing the levels of protein phosphorylation, such as IR, IRS-1 and Akt, and reduction of IRS- 1 ser307 phosphorylation levels. As insulin resistance leads to the progression of obesity to type-2 diabetes, Chlorella has a beneficial effect in the direct prevention of the disease. In addition, improvements in the quality of life, the mood and the disposition of chronic Chlorella users have been observed. Type-2 diabetes is related to high mortality, morbidity and poor general condition of the patient, thus leading to a decrease in quality of life. Thus, adaptogenic algae may, in addition to having direct action in the evolution of the disease, improve the health of the patient as a whole. In this sense, the impact of the use of Chlorella on patients' quality of life was evaluated using the Short Form-36 Health Survey (SF-36), which provides practical information on the patient's health functional and your well-being. And the amounts of IL-6, IL-10 and TNFalpha cytokines of the patients were analyzed. The results showed a significant improvement in quality of life after the use of algae in the evaluated patients (type-2 diabetics, glucose intolerance and control). Regarding the cytokines, IL- 6 and TNF- $\alpha$ showed decrease and IL-10 showed increase after the use of Chlorella. In these studies, there was the financial support of PIBIC / UNICAMP, FAEPEX, FAPESP, CNPq.

\section{CANNABINOID SYSTEM AND SCHIZOPHRENIA: A CONTEXTUALIZATION.}

Carolina Galvão Salioni, Alicia Arioli Mauro, Priscila De Freitas Lima.

Through a brief literature review the present study aims to contextualize the use of Cannabis to the symptoms of schizophrenia. Interest in the supposed relationship is due to the fact that the human organism has $\mathrm{CB}_{1}$ and $\mathrm{CB}_{2}$ receptors for cannabinoids, including the phytocannabinoid 0 tetrahydrocannabinol, the active principle of the Cannabis plant. Evidence suggests that there are alleles that increase the risk of schizophrenia through Cannabis use. In addition, the binding of of the before mentioned phytocannabinoid to such receptors also enhances the manifestation of psychotic symptoms in healthy people, as well as the exacerbation of such symptoms in chronic users of the plant. Individuals who do not have schizophrenia, but with a greater susceptibility to such disease due to the presence of genetic factors, are exposed to the acceleration of the manifestation of this psychosis if there is a frequent use of Cannabis. Canabidiol, another cannabinoid also present in the plant, if used in isolation, can supposedly attenuate the anxiety and certain behavioral effects produced by high doses of delta 9-tetrahydrocannabinol. Even with evidence pointing to possible relationships between cannabinoids and schizophrenia, there are still points that can be discussed, such as the fact that new cases of schizophrenia are not equated with increased use of Cannabis worldwide. Thus, although there are different views and studies about the possible relationship between the Cannabis plant and schizophrenia, there is evidence that phytocannabinoids such as delta 9tetrahydrocannabinol induce psychotic symptoms in healthy people and exacerbate the symptoms of patients already diagnosed with schizophrenia.

79. THE ANTITUMOR EFFECT OF CAFFEINE ON GLIOBLASTOMA CELL LINES.

Matheus Negri Boschiero, Gabriel Alves Bonafé, Bruno Camporeze, Manoela Marques Ortega.

INTRODUCTION: Cliomas are the most common brain cancers, comprising of about $50 \%$ of malignant brain tumors in adults. Grade IV glioma, known as glioblastoma (CB), is the most frequent and agressive neurologic tumor representing about $80 \%$ of glioma cases. According to a study, high and daily doses of caffeine can lower riso of GB developement by up to $30 \%$.
Caffeine is known as a neuroactive compound with anti-inflammatory and antioxidant effects. DISCUSSION: The caffeine provided lowered activity of the protein deacetylase $\mathrm{H}_{1}\left(\mathrm{HDA}_{1}\right)$ and increased activity of the protein acetyltransferase $\mathrm{p} 300$, both of which are involved in histones acetylation. This imbalance of $\mathrm{HDA}_{1} / \mathrm{p}_{300}$ resulted in cell death. Furthermore, the caffeine stopped the cell cycle during the $\mathrm{Co}_{\mathrm{C}} \mathrm{G}_{1}$ phase, decreasing the number of cells in the $S$ phase and reducing the $C B$ in mouse and human cell lines (C6 and U87MG, respectively). Moreover, it can disassociate the cytocrome $C$, leading to caspase mediated apoptosis, or causing the hyperexpression of FOX01, which carries greater accumulation of the Foxo1 protein, which can stimulate Bim, activating caspases mediated apoptosis. The phenethyl ester of cafeic acid (CAPE), derivative of coffee, is associated with the inhibition of the human GB cell line (T98G) proliferation, and it has shown citotoxicity in high concentrations in the human CB cell lines T98G, U87MG, U251. The inhibitor of IL17A, named FLVM (CAPE derivative), can decrease the tumour growth by reducing of HIF-1 $\alpha$, angiogenesis, VECF, IL17A and cell proliferation, consequently and significantly increasing Bax, caspases and FasL. CONCLUSION: When introduced, caffeine can inhibit cell proliferation, invasion and angiogenesis. Also, it can activate apoptosis in the studied GB human cell lines.

\section{CURCUMIN TREATMENT IN VITRO AND IN VIVO EFFECTS ON ACUTE MYELOID LEUKAEMIA.}

Henriquy Aguiar Coelho, Marisa Claudia Alvarez de Prax, Irene Santos, Karla Priscilla Vieira, Sara Teresinha Olalla Saad.

INTRODUCTION: The curcumin, one of the substances that can be extracted from turmeric - plant of extreme phytotherapic relevance -, has been broadly studied, and some positive effects such as mitigating the growth of tumours, have already been proved. Acute myeloid leukaemia (AML) is an aggressive hematological disorder, whose development and progression envolve a deregulation of the cellular apoptosis. OBJECTIVE: evaluate the effects of curcumin in vitro and in vivo, through a xenograft tumoural model. Material and methods: the cellular viability of the U937 lineage was determined by the MTT's method and the apoptosis by flow cytometry, through propidum iodide $(\mathrm{PI})$ and Anexina $\mathrm{V}$ - FITC readings. The determination of autophagic potencial mediated by curcumin using acridine orange as a fluorescence emitter for detection on the flow cytometer. To quantificate reactive oxigen species was used DCFDA as marker. The in vivo studies were carried out in xenograft tumoural model from the U937 lineage. RESULTS: the in vitro tests have shown that the treatment with curcumin induces apoptosis for the tested concentrations and its percentage is dependant dose, being it $50 \pm 6,2 \%$ for $25 \mathrm{uM}$ of curcumin. In the release of ROS, curcumin has a pro-oxidant character. In the autophagic process, it presents an important increase of acid vesicles, indicating a possible active autophagic process, being $25 \mathrm{uM}$ after $48 \mathrm{~h}$ the ideal concentration. Furthermore, the in vivo testings, have shown that curcumin inhibit the tumoural growth. The sample (consisted of a curcumin dose with a mass concentration of $100 \mathrm{mg} / \mathrm{kg}$, intraperitoneal, alternating the days) showed a tumoural reduction of $46 \%$ for the group treated with polyethyleneglycol as solvent and $61 \%$ for the group treated with curcumin encapsulated in Pluronic mixed micelles. DISCUSSION: the results confirmed the ones previously found in literature, verifying the antineoplasic potential for solid tumors besides the potential for the treatment of neoplasms derived from hematopoiéticas lineages. CONCLUSION: the curcumin, in the tested doses, induces apoptosis, ROS release and autophagic process. In vivo, the composite inhibit the tumoural growth which could be proved by the tumoural expansion slowing down, seen in xenograft model carried out in mouses NOD/SCID.

\section{ANTI-HISTAMINE EFFECT OF JAMBU.}

Thuany Caroliny Coelho, Adriana Rodrigues dos Anjos Mendonça, Carolina Conçalves Andrade, Ana Beatriz Alkimin Teixeira Loyola.

INTRODUCTION: Alternative and Complementary Medicine (CAM) has been increasingly used by citizens around the world as a means to improve and treat their health. According to EHTPA, herbal medicine is one of the oldest known forms of treatment and the medicinal use of plants is common in all cultures and peoples of the world. Acmella oleracea (L.) RKJansen (Spilanthes oleracea, syn. Spilanthes acmella var. Oleracea) is a plant of the Asteracea family popularly known as "jambu", "bracken watercress" or "watercress from Pará". Evaluate the antihistaminic effect of Jambú. METHOD: The present study was developed at Botany Laboratories of the Sapucaí Valley University (Univás), Pouso Alegre - MG. It is a cross-sectional, 
individual controlled, analytical and observational study. 49 volunteers participated in the study. Each volunteer, after signing the Term of Free and Informed Consent, answered a questionnaire with information about their personal characteristics and did the Prick test, which is the most commonly used skin test. The individuals in whom this test was applied will be distributed in a single experimental group and each individual will be its own control (paired sample). All 49 sampled individuals will have their forearm adequately prepared for the procedure and sensitized at three points (points $A, B, C$ ). Point $A$ will be the positive control: sensitizing with histamine, point $B$ will be the negative control: by applying only the jambú, the point $C$ will be sensitized with histamine and the jambú will be applied instantaneously. RESULTS: After collecting the data, the median of points A and $C$ were, respectively, 3.4730 and 3.2210. While at point $B$ it was 0.000 . The analysis of variance of Friedman corresponds to 61,19 with $p<0.0001$. CONCLUSION: Jambú did not present antihistaminic activity.

82. NON-TUBERCULOSIS MYCOBACTERIA: MICROBIOLOGICAL DATA AND CLINICAL PROFILE OF PATIENTS WITH POSITIVE ISOLATES IN A UNIVERSITY HOSPITAL IN THE CITY OF CAMPINAS, STATE OF SÃO PAULO, BRAZIL.

Nathalia Fernanda Nora Santos, Mônica Corso Pereira.

The frequency of non-tuberculous mycobacteria (NTM) infections outpaces mycobacteria tuberculosis (TB) infections in developed countries, and in recent decades the isolation of these pathogens has been increasing. Although infection can occur in almost any organ, lung infections are the most common. M. avium, M. kansasii and M. abscessus are the most frequently identified microorganisms causing lung disease. These microorganisms can be found in soil and water and, although the specific source of infection generally can not be identified, human disease is suspected to be acquired from environmental exposures. Isolation of an MNT does not necessarily mean active infection. Clinical, radiological and microbiological parameters are necessary to establish the diagnosis of active infection (or disease) by NTM. Although these infections are becoming more and more common in clinical practice, there are still significant gaps in knowledge about the epidemiological, clinical and laboratory aspects of these microorganisms, making new studies on the subject essential. Theoretically the therapeutic management of these patients (pulmonary infections by NTM) must follow the international recommendations (American Thoracic Society) and national (Ministry of Health) recommendations. Raising information about how in practice patients are being treated in a referral center will provide relevant data. The objective of this research is to know the clinical, microbiological, laboratory and radiological aspects of patients with at least one positive isolate for NTM (especially those with pulmonary infections) in a university hospital Unicamp Hospital de Clínicas, a tertiary complexity care center that possesses (tuberculosis) and non-tuberculosis (NTM). It is expected that the knowledge of the spectrum of the clinical presentations of the infection disease by NTM and the treatment of cases considered as disease will help to improve the diagnostic approach and the therapeutic management of patients with these conditions.

83. THE RELATIONSHIP BETWEEN NEUROPSYCHOLOGICAL TESTS AND CEREBRAL SPINAL FLUID LEVELS OF AMYLOID BETA AND TAU PROTEINS WITH THE WHITE MATTER INTEGRITY IN THE ALZHEIMER'S DISEASE.

Christian Luiz Baptista Gerbelli, Thamires Naela Cardoso Magalhães, Marcio Luiz Figueredo Balthazar.

BACKGROUND: The dementia due to Alzheimer's disease (AD) is a neurodegenerative disorder through different pathophysiological ways promotes cognitive and neuropsychiatric alterations restricting the functional independence of the subject. The pathophysiology evolves essentially two proteins: amyloid (A) and tau proteins. OBJECTIVES: to evaluate the relationship between neuropsychological tests (NT) and cerebrospinal fluid biomarkers of $A D$, i.e. $A$, total-tau (t-tau) and phosphorylated-tau ( $\mathrm{p}$-tau) with white matter (WM) integrity of whole brain evaluated by diffusion tensor imaging (DTI) technique in patients with mild $A D$ and amnestic mild cognitive impairment (aMCI). METHODS: 62 patients with $\mathrm{aMCl}, 30$ with mild $\mathrm{AD}$ and 105 controls were involved. All participants underwent broad neuropsychological evaluation and brain Magnetic Resonance Imaging (MRI) in $3 \mathrm{~T}$ scanner. $\mathrm{AD}$ and $\mathrm{aMCl}$ subjects underwent lumbar puncture to analyze $A \beta, p$-tau and t-tau levels. To analyze WM integrity, we used an automated segmentation method - MultiAtlas, which evaluates DTI data and extract fractional anisotropy (FA), radial diffusivity
(RD), mean diffusivity (MD) and axial diffusivity (AD) values. The DTI values were correlated with CSF levels and NT. RESULTS: p-tau and t-tau levels correlated with values of FA on right fornix. Moreover, values of FA, RD and MD were significantly different between controls and $\mathrm{aMCl}$ patients Furthermore, several correlations were obtained between NT and DT parameters. CONCLUSION: p-tau and t-tau proteins may be responsible for the propagation and perpetuation of the AD dementia, while the $A \beta$ proteins don't have a prominent role in this process. Regions with abnormal values of FA, RD and MD may take part of the early stages of $A D$ dementia. And the damage in the WM microstructure of the brain is related to the patient's clinical manifestation, evaluated by several NT.

\section{SPONDIAS MOMBIN SUPPLEMENTATION ATTENUATED CARDIAC} REMODELLING PROCESS INDUCED BY TOBACCO SMOKE.

Elisa Moya Kazmarek, Maísa Ayumi Kimura, Marcos Ferreira Minicucci.

The objective of this study was to investigate the influence of Spondias mombin (SM) supplementation on the cardiac remodelling process induced by exposure to tobacco smoke (ETS) in rats. Male Wistar rats were divided into 4 groups: group $\mathrm{C}$ (control, $\mathrm{n}=20$ ) comprised animals not exposed to cigarette smoke and received standard chow; group ETS $(n=20)$ comprised animals exposed to cigarette smoke and received standard chow; group ETS100 $(n=20)$ received standard chow supplemented with $100 \mathrm{mg} / \mathrm{kg}$ body weight/d of SM; and group ETS250 $(n=20)$ received standard chow supplemented with $250 \mathrm{mg} / \mathrm{kg}$ body weight/d of SM. The observation period was 2 months. The ETS animals had higher values of left cardiac chamber diameters and of left ventricular mass index. SM supplementation attenuated these changes. In addition, the myocyte cross-sectional area (CSA) was lower in group C compared with the ETS groups; however, the ETS250 group had lower values of CSA compared with the ETS group. The ETS group also showed higher cardiac levels of lipid hydroperoxide ( $\mathrm{LH}$ ) compared with group C; and, groups ETS100 and ETS250 had lowe concentrations of LH compared with the ETS group. Regarding energy metabolism, SM supplementation decreased glycolysis and increased the $\beta$ oxidation and the oxidative phosphorylation. In conclusion, our results suggest that ETS induced the cardiac remodelling process. In addition, SM supplementation attenuated this process, along with oxidative stress reduction and energy metabolism modulation.

\section{CEREBELLAR ATROPHY IN NEUROMYELITIS OPTICA SPECTRUM DISEASE (NMOSD) PATIENTS.}

Rodrigo Francisco da Silva Souza, Clarissa Lin Yasuda.

INTRODUCTION: Neuromyelitis Optica Spectrum Disease (NMOSD) is an autoimmune neurodegenerative process which affects the optic nerve and spinal cord in inflammatory outbreaks, leading to its symptons 1 . Newly, recent studies have showed that other structures in central nervous system (CNS), like the brain cortex, thalamus and medulla oblongata are affected to02,3. The increased use of different techniques of magnetic resonance imaging (MRI) analysis, such the voxel-based morphometry (VBM), has improved our knowledge about the real CNS damage in NMOSD patients. MATERIALS AND METHODS: Thirty-nine NMOSD AQP4-IgG positive patients from UNICAMP's Neurology Service were selected to participate in this study: fifteen of these patients presented neuromyelitis optica (NMO), nine presented longitudinally extensive transverse myelitis (LETM) and eight are diagnosed with optic neuritis only. For group comparisons, forty-two healthy controls were selected from an MRI bank of healthy volunteers. Images were acquired at Hospital de Clínicas (UNICAMP) using a 3 Tesla Achieva-Intera Philips MRI scanner including 3D-T1 weighted images (isotropic voxels of 1 $\mathrm{mm} 3$, acquired in the sagittal plane; $1 \mathrm{~mm}$ thick, flip angle $=80, T R=7 \mathrm{~ms}$, $\mathrm{TE} 3,2 \mathrm{~ms}, \mathrm{FOV}=240 \times 240 \times 180 \mathrm{~mm} 3)$. All images were segmented according to standard SPM12/CAT12 and SUIT protocols (http://www.neuro.unijena.de/cat/) (www.fil.ion.ucl.ac.uk) (http://www.diedrichsenlab.org/imaging/suit.htm), which included: spatial normalization, cerebellum segmentation and smoothing, Quality control of image segmentation was performed. Statistical analysis of images was performed with SPM12, while clinical information was compared with GraphPad Prism 7. RESULTS: NMOSD and control were paired by gender and age. The figure below shows significant cerebellar cortical atrophy in NMOSD patients compared to healthy controls $(p<0.05)$. DISCUSSION: Cerebellar cortical atrophy observed in patients group confirms the recent studies results', in which other areas of CNS than the classic ones are affected by NMOSD. It is possible that these findings occurs due to direct 
immunomediated lesions or by a secondary degeneration mechanism in areas interconnected in the CNS. CONCLUSION: Cerebellar atrophy can be seen in NMOSD patients, especially in the ones who present LETM or NMO spectrum of the disease. Further studies of clinical symptoms in these patients or using other MRI techniques may reveal the complete clinical meaning of these findings.

\section{INFLUENCE OF TP53 Arg72PRO POLYMORPHISM ON THE RISK OF} LARYNGEAL SQUAMOUS CELL CARCINOMA.

Letícia Kimie Murazawa, Ricardo Afonso Alves dos Santos, Gustavo Jacob Lourenço, Carmen Silvia Passos Lima.

The laryngeal squamous cell carcinoma (LSCC) has high rates of incidence, mortality and therapeutic consequences. Abnormalities in genes involved in DNA damage repair and in apoptosis induction of cell whose DNA cannot be repaired may be associated with the occurrence of LSCC. The TP53 Arg72Pro polymorphism varies in ability of inducing apoptosis, and the Pro allele are less efficient than the Arg allele in the process. The role of this polymorphism in the risk of LSCC, as well as in clinical and biological manifestations of the tumour is unknown; so these were the objectives of this study. We studied 203 patients with LSCC treated in the Clinical Oncology Service of the University of Campinas Teaching Hospital and 207 blood donors seen at University of Campinas Blood Center. The controls were matched to patients by gender and ethnicity. The genotypes were identified using the real-time polymerase chain reaction in DNA obtained from peripheral blood samples. Differences between groups were analyzed by Fisher test and chi-square, and multiple logistic regression for odds ratio (OR) and $95 \%$ confidence intervals. Controls' samples were in HardyWeinberg equilibrium (HW) at the $\mathrm{TP}_{53}$ Arg72Pro polymorphism locus ( $\mathrm{\chi}_{2}=$ $0.027, P=0.86)$, while patients' samples were in disequilibrium $(\chi 2=11.87$, $P<0.01)$. Similar frequencies of polymorphism genotypes were seen in patients and controls. However when evaluated genotypes of patients stratified according to the clinical aspects, there were a higher frequency of ArgPro+ProPro genotype in nonwhite skin patients than that observed in white skin patients $(64.6 \%$ versus $40.6 \%, P=0.005 ; P A=83.9 \%)$. No significant differences were found in patients stratified by genotypes and other clinical and tumour characteristics. This data suggest that different genotypes of TP53 Arg72Pro polymorphism do not alter the risk of LSCC in general population. However, ArgPro+ProPro genotype may be associated with tumour occurrence in non-white individuals.

87.

Flávio Augusto Barbieri, Amanda Cristina Tassi, Giulia Christiano Ferreira de Oliveira.

Fibromyalgia is a musculoskeletal disorder of rheumatological scope, characterized mainly by chronic and diffuse pain, without anatomical substrate that explains its pathophysiology, Meanwhile Persistent Somatoform Pain Disorder (PSPD) is a category of somatoform disorder characterized by psychogenic and chronic pain, unlinked from organic substrates that suggest it. The fact that both diseases have the same focus in the clinical feature, chronic pain, leads to relatively similar diagnostic criteria, this evidencing the possibility of the same patient conferring diagnoses for both diseases. This is a descriptive study with a quantitative approach in which the Diagnostic Criteria for Fibromyalgia, as suggested by the American College of Rheumatology in 2010, and Diagnostic Questionnaire for Persistent Somatoform Pain Disorder based on the criteria established by the Diagnostic and Statistical Manual of Disorders Mental IV (DSM-IV). These were administered on a sample of 20 patients, randomly selected, between 30 and 75 years, diagnosed with fibromyalgia in the NGA3 of Araraquara, where they undergo clinical follow-up. After the collection and analysis of the results, it was observed that the sample was composed entirely of the female gender and that $95 \%$ of the patients gave at least 4 of the 5 diagnostic criteria for DSM-IV PSPD. When analyzing these results, there was a correlation between the generalized pain index and the PSPD, and the higher the pain index, the more similar to the PSPD patient the individual presented. By analyzing the diagnostic criteria of both diseases, it is possible to conclude that these, clinically, are the same condition.

88. SUBTENONIAN AND PERIBULBAR BLOCK FOR CATARACT SURGERY: A SYSTEMATIC REVIEW.

Jose Helio Zen Junior, Carlos Eduardo Leite Arieta, Fabio Nero Mitsuushi.
The techniques of ophthalmological anesthesia day after day present more indications in front of the constant increase of the ophthalmological attention in the world. Among the anesthetic techniques are ophthalmic blockages. The present study seeks to perform a systematic review comparing two techniques, subtenonian and peribulbar block, already diffused in several places and that dispute preferences among the specialists regarding the quality of the anesthesia and comfort of the patient and the surgeon.

89.

THE INCIDENCE OF NEUROCRYPTOCOCCOSIS IN PATIENTS WHO HAVE BEEN ATTENDED AT THE HOSPITAL DAS CLÍNICAS DA UNICAMP/SP AND THE EFFICIENCY OF DIAGNOSTIC TECHNIQUES.

Leonardo Amaro Alexandre de Almeida, Célia Regina Garlipp.

Cryptococcosis is a mycosis caused by the fungi of the Cryptococcus neoformans species complex and it is strongly associated with immunocompromised HIV patients. The Cryptococcosis infection begins in the respiratory system and it progresses to a meningoencephalitis, the most common form of neurological manifestation of the infection, which has a chronic disease process. Our aim was to analyse within the period of 6 (six) years - from $01 / 2012$ to $01 / 2017$ - the incidence of neurocryptococcosis in the Hospital de Clínicas da Unicamp/SP and evaluate the efficiency of three available laboratory techniques for the tracing of Cryptococcus $\mathrm{sp}$ in the cerebrospinal fluid (CSF), (named: direct investigation without staining; India ink stain and latex agglutination) against culture examination for Cryptococcus which is considered the gold standard one. Taking into account the 9572 cerebrospinal fluid (CSF) samples collected in the established period we could observe an average frequency of 9 diagnosed cases / year, and at the end of the study 53 cerebrospinal fluid (CSF) samples were identified from 53 patients with confirmed diagnosis of neurocryptococcosis. Most of those patients were male ones $(64 \%)$, HIV positive $(40 \%)$, aging from 39 to 49 years old $(66 \%)$. In 41 samples $(77 \%)$ the three diagnostic techniques, that were the object of the study, were carried out in addition to the culture one, and in only one sample there was a divergence between the results obtained. The culture technique, aiming at the identification of the Cryptococcus, was carried out in $98 \%$ (52) of the samples, Cryptococcus neoformans was isolated in $77 \%$ (40) of the positive cultures whereas Cryptococcus gattii was isolated in $13 \%$ (6) of the cerebrospinal fluid samples. Considering the samples with negative culture (6) in 5 of them the three techniques were positive for the presence of fungi, while in the other sample only two techniques were used (direct fungi research and Indian ink) also identifying the presence of fungi. The combined use of those three diagnostic techniques was proved effective in the identification of Cryptococcus sp, as they made it possible to identify $100 \%$ of patients with neurocryptococcosis. Therefore, the obtained result allows us to assure that the answers provided by the diagnostic methods investigated are reliable and allow a safe diagnosis of the disease.

\section{EVALUATION OF COGNITIVE IMPAIRMENT IN PATIENTS WITH EPISODIC MIGRAINE.}

Caroliny Trevisan Teixeira, Carolina Minarro Cremasco, Anelise Melo, Carolina Cáfaro, Karen dos Santos Ferreira.

BACKGROUND AND OBJECTIVES: The purpose of this study was to evaluate the presence of cognitive deficits in patients with episodic migraine and control group, and to compare with the results previously described in the literature. METHODS: Patients with episodic migraine were included according to the International Headache Society criteria, and a control group was selected, matched by sex, age, and schooling. Patients were asked about the frequency and intensity of headaches, medications used, and comorbidities. Afterwards, a neuropsychological assessment was applied using the Montreal Cognitive Assessment, the clock drawing test, the verbal fluency test, and the Stroop test. The main variables influencing cognitive dysfunctions were analyzed (depressive disorder, anxiety disorder, sleep disorder, use of medication). RESULTS: Thirty patients with episodic migraine and 30 controls were evaluated. Patients with breakthrough migraine had a performance in the Montreal Cognitive Assessment test $(p=0.53)$, the clock drawing test $(p=0.80)$, the verbal fluency test $(p=0.44)$ and the Stroop test $(p=0.97)$ similar to the control group. Patients with chronic migraine (30 patients and 30 controls) performed a Montreal Cognitive Assessment test $(p=0.00)$, verbal fluency test $(p=0.00)$ and Stroop test $(p=0.00)$ lower than the group control. The main variables influencing cognitive disorders were studied by linear regression, and none of these variables was an influencing factor in the Montreal Cognitive Assessment test. CONCLUSION: Patients with 
breakthrough migraine did not present cognitive deficits compared to a control group.

\section{PARANEOPLASTIC SYNDROMES THAT MANIFEST THEMSELVES AS PAINFUL NEUROPATHIES}

Luciana Guimarães Caixeta, Paula Zuliani Aquatti, Karen dos Santos Ferreira.

PURPOSE/ AIM OF THE STUDY: Paraneoplastic syndromes (PSs) are described as disorders from an indirect tumor mechanism, other than a metastatic or a neoplastic infiltration, which can affect several systems. Paraneoplastic syndromes that manifest as neuropathies present themselves in a varied way, with a common denominator: pain. Such pathologies have their physiopatological aspects little elucidated in a systematic way. Thus, this study aims to systematically review the main studies involving SPs that manifest as painful neuropathies. METHODS: We reviewed studies that met certain criteria by searching Pubmed, Ebsco host, and Cochrane Systematic Reviews Database, using the words "paraneoplastic" OR "paraneoplastic syndrome" OR "paraneoplastic polyneuropathy", AND "neuropathy", AND "pain". No limits were applied for language or publication time. RESULTS: Paraneoplastic syndromes that manifest as painful neuropathies include sensory-motor neuropathies, optic neuropathies, mononeuropathies, autonomic neuropathies, Guillain Barré syndrome, and other inflammatory polyradiculopathies. Pain in these neuropathies assumes varied characteristics, as burning and dysesthesic characteristics, radiculopathic pain, possibly in the lumbar region, radiating to the lower limbs, periorbital pain in optic neuropathies, pain accompanied by autonomic signs compatible with Regional Complex Pain Syndrome, gastrointestinal and esophageal dysmotility pain in autonomic neuropathies, or simulating nociceptive pain. CONCLUSION: The present study reviews concepts about Paraneoplastic Syndromes that manifest as painful neuropathies, describing possible diagnoses and clinical manifestations.

92.

THE INFLUENCE OF PRO-INFLAMMATORY FACTORS AND PLASMA LIPIDS ON THE PHYSIOPATHOLOGY OF HEART FAILURE.

Rafaela Seraphim Frare, Nágila Emmanoele Bernardo da Silva, Gabriel Ramon Matavelli Casseb.

BACKGROUND: It is known that cardiovascular diseases, such as heart failure (HF), are one of the leading causes of death in the world. In 2015, the World Health Organization has estimated that 23 million people worldwide suffer from HF, with cancer-equivalent mortality rates. Thus, the study about mechanisms capable of influencing this pathology is important insofar as its prevalence remains high even with advances in health. It has been observed that considerable levels of HDLcholesterol have been associated with greater survival in $\mathrm{HF}$, so its several protective functions must be effectively explored. METHODS: A literature review was carried out based on scientific articles researched in PubMed, LILACS, BIREME and SciELO on the subject "The influence of pro-inflammatory factors and plasma lipids on the physiopathology of heart failure". RESULTS: It was observed that in patient with more severe and symptomatic HF, the process of lipid transfer to HD is deficient, as well as some of the mechanisms regulating it, and possibly these alterations influence the reverse transport of cholesterol and on the protective functions of HDL in these patients. DISCUSSION: Studies have shown that transfer of lipids to HDL is altered by a number of conditions, such as coronary artery disease, diabetes mellitus and sedentary lifestyle. Recently, HDL has been reported to carry small non-protein encoding RNAs, the so-called microRNAs (miRNAs). Some miRNAs have been described as essential regulators of lipoprotein metabolism, and possible changes in this transport may influence HF. Thus, we aimed to evaluate in this review study: 1) Transfer of lipids to HDL; 2) Expression of circulating miRNAs involved in the reverse transport of cholesterol; 3) Correlation between functional aspects of $\mathrm{HDL}$ with predictive inflammatory factors of $\mathrm{HF}$.

93.

LEUKEMIA - THE IMPORTANCE OF INTEGRATED CARE AND THE ROLE OF THE OPHTHALMOLOGIST.

Samuel Serpa Steck, Juliana Fonseca Serpa Steck.

Here reported is a case that demonstrates the importance of the multidisciplinary approach, which includes the ophthalmologic care in patients with diagnosis of leukemia. A 29-year-old patient diagnosed with acute lymphoblastic leukemia for 7 months presented ocular manifestations associated with the primary disease. The incidence of ocular manifestations in leukemia is independent of the cytologic type and the course (acute or chronic), ranging from 10 to $90 \%$ of patients. Among these, 15 to $55 \%$ present some type of ocular manifestation before the start of chemotherapy. Although most patients are already diagnosed with leukemia prior to ophthalmologic evaluation, ocular symptoms may, even if rarely, precede the primary diagnosis or suggest a diagnosis of relapse or complications of the disease or treatment. Retinal hemorrhage, usually caused by severe hemorrhage ( $\mathrm{Hb}$ less than $6 \mathrm{~g} / \mathrm{dL}$ ) and thrombocytopenia, is the most frequent finding in the ocular examination, usually associated with complaints of poor visual acuity and improvement with treatment of the systemic disease. Increased disease survival, associated with a higher incidence of ocular symptoms and the lack of definitive consensus in the literature on the relationship between ocular involvement and prognosis demonstrate the need for studies regarding such issues. The case reported here corroborates the association between ocular involvement and worse prognosis.

94. COMPARISON OF PULMONARY ULTRASONOGRAPHY FINDINGS AND HIGH-RESOLUTION COMPUTERIZED THORAX TOMOGRAPHY WITH THE PULMONARY FUNCTION OF CIRRHOTIC PATIENTS WITH INDICATION OF HEPATIC TRANSPLANTATION.

Augusto César Bortoluci, Luiz Claudio Martins.

In 2016, the number of indications for liver transplantation reached 5.111 people in Brazil, with 1.880 interventions being performed. During the waiting period for transplantation, patients undergo constant follow-ups to monitor their health status and evaluate comorbidities that develop due to liver dysfunctions, and thus, are submitted to different exams and interventions. As part of this follow-ups, we have pulmonary evaluation standardized through pulmonary function tests by spirometry, and highresolution computed tomography of the lungs. Despite the high definition of lung parenchyma imaging, such examination is invasive and presents risks such as repeated exposure to high doses of radiation. On the other hand, the use of pulmonary ultrasonography has been expanding since the beginning of the 1990s, and was then introduced in routine pulmonary emergency assessment, with specific imaging patterns already established in the literature. The use of this technique in the evaluation of critical patients such as patients in the transplant queue is still unde development, and is not part of the routine. Therefore, there is no scientific data comparing the findings of the gold standard examination with ultrasound. The purpose of this study was to compare the findings of pulmonary ultrasonography in ambulatory patients undergoing follow-ups at Hospital das Clínicas of UNICAMP with high resolution computed tomography scans. Besides that, we had compared he correspondence of the signals in both methods image with the findings of the pulmonary function test (spirometry), in the hope of establishing which imaging method best correlates with pulmonary functional alterations. From the comparison of the findings, we had a concordance with the demographic values of the sample in relation to the distribution by sex ( 43 people $-76.8 \%$ male), age $(56 \pm 8,13), \operatorname{BMI}(28.77 \pm 6,11)$, MELD $(16,11 \pm 6,22)$ and also with the cause of the indication of transplantation being predominant the infection by the virus $C$ and ethylism. The spirometry test had no alterations in the selected sample, and the patients were in the expected range for sex and age, despite the baseline condition. The results of the tomography showed that $11.1 \%$ of the patients presented bronchial syndromes, $70 \%$ demonstrated pulmonary syndromes and any patient has had lauded the diagnosis of pleural syndrome. When using ultrasonography, none presented a diagnosis of bronchial syndrome, $70 \%$ presented $B$ lines (equivalent to pulmonary syndrome by TC), and $11.1 \%$ had pleura syndromes. With this data, it was concluded that, in cirrhotic patients, ultrasonography is a complementary examination to tomography in the care of patients, mainly in the detection of pulmonary syndromes, especially for pleural effusion of small volume.

95. OUTCOME OF TREATMENT WITH THIONAMIDES AND ITS ADVERSE EFFECTS IN PATIENTS WITH GRAVES' DISEASE: RELATIONSHIP WITH DEMOGRAPHIC AND DISEASE'S FEATURES.

Isabella Lucca de Campos Lima, Isabela Sayuri Okuma, Denise Engelbrecht Zantut-Wittmann, Marcos Antonio Tambascia, Arnaldo Moura Neto.

Graves' disease is an autoimmune disease and is the main cause of hyperthyroidism, affecting mainly women in the age range of 40-60 years. The pathogenesis involves the presence of anti-TSH receptor antibodies 
(TRAb), which induce continuous and uncontrolled thyroid stimulation, which leads to excessive synthesis of thyroid hormones and to thyroid hypertrophy. The choice treatment is performed with antithyroid drugs, and radioiodine or thyroidectomy may be used in case of therapeutic failure. This study aims to characterize the evolution of treatment with antithyroid drugs as well as its adverse effects in patients with Graves' disease. A review was conducted of the medical records of 251 patients diagnosed with Graves' disease, of both sexes, followed in Thyroid Dysfunction Clinic of the Endocrinology Division of HC-Unicamp, with subsequent descriptive data analysis, with position and dispersion measurements for continuous variables and frequency tables for categorical variables. There were analyzed variables such as sex, race, age, age at diagnosis, family history, smoking, alcoholism, ophthalmopathy, presence of antithyroid antibodies, morphological changes to ultrasonography, thyrotropin (TSH) and free thyroxine ( $\left.\mathrm{FT}_{4}\right)$, thyroid scintigraphy, drug, dose and duration of treatment. of the patients, $78.09 \%$ were women, $73.88 \%$ were white, mean age was 49.93 years and mean age at diagnosis was 38.90 years. Regarding drug treatment, $92.8 \%$ were treated with methimazole and $6.8 \%$ with propylthiouracil. Methimazole doses ranged from 5 to $60 \mathrm{mg}$ daily, with a mean of $29.28 \mathrm{mg}$; the propylthiouracil doses ranged from 100 to $800 \mathrm{mg}$ per day, with a mean of $364.70 \mathrm{mg}$. The mean duration of treatment was 53.12 months. During treatment, $11.16 \%$ of the patients had adverse effects, $61.5 \%$ of which were to methimazole, and $38.5 \%$ to propylthiouracil, represented in Chart 1. The thionamide type was the main determinant, among the analyzed variables, for the appearance of adverse effects ( $p<0.0001)$. The use of propylthiouracil reflected a 19.286 times greater chance of having some adverse effect in relation to the use of methimazole. The evolution after treatment with thionamides showed remission of the disease in $20.3 \%$ of the cases, considering that the study was performed in a tertiary hospital, in which the majority of the cases are those of greater complexity, which do not had a good response to primary care treatment. Thus, $79.7 \%$ of the total sample showed no remission with thionamide, being referred to other types of treatment. Among the variables in relation to the evolution of treatment, TRAb $(p=0.0002)$ in high concentrations indicated a 5.211 times greater chance of non-remission of the disease with the use of thionamides.

\section{NS1 ANTIGEN FOR DIAGNOSIS OF DENGUE AND POTENTIAL CORRELATIONS WITH COMPLICATED DENGUE. \\ Telma Maria Moreira, Francisco Hideo Aoki.}

DENV-1, DENV-2, DENV-3 and DENV-4, all belonging to the genus Flaviviruse transmitted by mosquitoes of the genus Aedes, are caused by acute febrile arbovirose, a latent public health concern caused by four different viral serotypes. Infection with any of these serotypes being symptomatic results in mild dengue, or more severe forms of the disease, such as dengue haemorrhagic fever (DHF) or dengue shock syndrome (DSS). Currently, five serological tests are used for diagnostic purposes, however, they are not as effective as they are either unresponsive, time-consuming, or lack specialized laboratories with cost-effective and well-trained professionals. In this sense, the NS1 antigen, a non-structural glycoprotein produced by all viral serotypes, has been studied as an alternative in the diagnosis of dengue, since it can not stimulate the human dengue fever, it is detectable from the first day to the next, without any serious symptoms, in addition to severe cases. This study aims to study serological results of NS1 and / or IgM and IgC for diagnosis of dengue and correlate them as severe forms of dengue. For this, clinical data will be compared and will be collected from the notification forms with the serological results of the examinations carried out by the LPAID.

\section{RELATIONSHIP BETWEEN BODY MASS AND LEFT VENTRICULAR MASS IN} CHRONIC RENAL PATIENTS.

Matheus Antônio Filiol Belin, Carlos Henrique Bianchi Florindo, Bianca Latance da Cruz, Tamires Baraviera Ukawa, Luis Cuadrado Martin.

INTRODUCTION: Chronic kidney disease (CKD) is a public health problem and a risk factor for cardiovascular disease (CVD) with high morbidity and mortality. Left ventricular hypertrophy (LVH) may be present in renal patients, contributing to CVD and increasing morbidity and mortality. Several factors may influence LVH, such as glomerular filtration, age, sex, ethnicity, blood pressure and overweight. OBJECTIVE: Thus, the aim of this study is to evaluate the association between body mass index and left ventricular mass index, in addition to other variables, in patients with nondialysis CKD. METHOD AND MATERIAL: In a cross-sectional observational study, 154 patients with CKD at Hospital das Clínicas de Botucatu (HCFMB) were evaluated for body mass index (BMI), age, gender, ethnicity, weight, height, creatinine, SBP and DBP by ABPM, mean FC and ventricular mass. The project was accepted by the ethics committee of FMB and a multiple linear regression was performed involving all evaluated variables, and results were discussed at a significance level of 0.05 . RESULTS: The mean age is $61.9 \pm 14.8$ years and 83 are males $(53.9 \%), 4$ are Afro-descendants, one Asian and the other whites. 107 (70\%) patients had ABPM with ambulatory hypertension. A significant positive and high magnitude linear correlation was observed between BMI $(r=0.431, p=0.000)$ and SBP 24h $(r$ $=0.193, p=0.006$ ) with LVH, however, this variable was not associated with the glomerular filtration rate. Systemic blood pressure and, especially, the body mass index of chronic non-dialytic renal patients are positively associated with left ventricular hypertrophy. CONCLUSION: Thus, it is important to adopt measures to reduce BMI and SBP in order to minimize cardiovascular complications from LVH in this population.

\section{DESCRIPTION AND ANALYSIS OF CANDIDEMIA EPISODES IN A TERTIARY HOSPITAL IN CAMPINAS (SP) BETWEEN 2009 AND 2018.}

Giovanna Barille, Thais Costa Reis Rodrigues, Rodrigo Otero Coelho, Maria Júlia Medrado Marques, André Giglio Bueno.

\section{Candidemia is defined as the isolation of any Candida species in at least} one blood culture and is currently an emerging challenge for hospitals. The knowledge of these data can improve the control of the levels of infections by this fungus, considering its increasing incidence and morbimortality. This study was conducted through the analysis of 189 cases that presented positive blood cultures for Candida spp, with or without signs of infection. Data were analyzed from 2009 to May 2018. Data such as age, comorbidities, risk factors, the Candida species, whether or not the fluconazole sensitivity test was performed, the treatment performed, the medication chosen, duration, use of descalamento and the outcome of the case. The main prevalent factors were: male sex (60.6\% of cases), older than 60 years ( $37.23 \%$ of cases), cardiovascular diseases $(55.87 \%)$, diabetes $(48.68 \%)$ and gastrointestinal diseases $46.67 \%$ ), previous use of antibiotics during hospitalization $(98.4 \%)$, presence of central venous catheter $(84.32 \%)$, mechanical ventilation ( $71.51 \%)$ and bladder catheter delay $(71.11 \%)$. The most commonly used antifungal was fluconazole (57.3\%), with $74 \%$ of the species being known to be sensitive, and the most common C. albicans species $(40 \%)$. As a result, $58 \%$ of the patients evolved to death. The results of the study confirmed the co-morbidities and widely explained risk factors as well as the more incident Candida species. Regarding treatment, that observed in the service does not necessarily follow the usual therapeutic practice. The study does not provide enough data to state that this has resulted in a lower cure rate, since systemic Candida infection has affected many critically ill patients.

\section{PARKINSON DISEASE: AGE-BASED INFLUENCE IN ASSOCIATION THE ADOPTION OF HEALTHY HABITS.}

Larissa Fernanda Fonseca Russo, Isadora Cabreira Góes, Marília Leal Escobar, Tainá Serena Mottin, Isabela Veiga Daud.

INTRODUCTION: Aging of the Central Nervous System affects a large part of the elderly and can lead to the appearance of certain neurodegenerative diseases, such as Parkinson's Disease. This disease is characterized by the destruction of dopaminergic neurons, leading to the reduction of dopamine in the central nervous system, resulting in certain motor alterations, which involve the slowing of movement sand the appearance of symptoms such as rest tremor, bradykinesia, stiffness and changes in posture and march. METHODOLOGY: DeCS site descriptors, articles analysis, case reports, data collection and bibliographic reviews found in SCIELO and PubMed sites were used to develop our bibliographic review work. RESULTS AND DISCUSSION: Studies show us that there are ways to prevent or less en the severity of this disease. It is believed that these changes are not only arising from the senile process, but also suffer a great influence of habits and lifestyles. A balanced diet combined with the frequent practice of physical exercises are some habits that allow the retardation or prevention of the development of degenerative neuropathies associated with a ging. CONCLUSIONS: Among the studies carried out, it is known that the best alternative to prevent the progression and onset of Parkinson's disease is throught head optionof healthy habits, such as physical exercise (particularly aerobics) and a balanced diet with energy restriction (caloric restriction) and reduction of stress level. In addition to this contribution related to Parkinson's disease, these actions also favor the improvement of the quality of life. 
100. LITERATURE REVIEW: BENEFITS OF PILATES IN PARKINSON'S DISEASE. Simone Aparecida Melo Soares, Lilian Cristina Faustinoni Campos, Jéssica Carniel Antonietti Chagas.

Parkinson's disease (PD) is a chronic degenerative disease with slow progression of the central nervous system (CNS) that causes the degeneration of dopaminergic cells of the substantia nigra. The rest train is the most characteristic sign of PD, with a unilateral involuntary movement in the distal part of the upper limb ("counting notes" movement). Bradykinesia, hypokinesia, akinesia, postural instability, plastic hypertonia, motor freezing and dysarthria are also common to the disease. As a disease that is not present, the treatment of PD aims to ease and delay the onset of symptoms and their progression. The Pilates method aims at preserving mobility, improving physical conditioning, maintaining muscle tone, gaining flexibility, postural corrections, balance and coordination, performing breathing exercises. The objective of this study was to present the benefits of the method for the patient with Parkinson's disease, thus accompanying the improvement of the quality of life and maintenance of the individual's functionality. It is a literary review study, based on the benefits to the person with Parkinson's disease. Included were those that show the importance of the practice of pilates in the treatment of PD. As databases used were: SciELO, Medline, Lilacs, Pubmed, PEdro in Portuguese, Spanish and English. Thirty - five articles were treated, but 27 were excluded, foramen 8 , but only 4 met the criteria. The Parkinson's Diseases. According to the data presented, the Pilates Method can contribute positively to the quality of patients with Parkinson's disease, being an effective and safe therapeutic resource. The best skills in balance, mobility, flexibility, strength, mobility, mobility and relaxation are the daily life activities of patients.

\section{ROUNDTABLE ON OBESITY.}

Carolina Magalhães Britto Rodrigues, Clarissa Mendes Remor, Débora Maria de Souza, Laíse Koenig de Lima, Rafaela Souza Reis.

The world and Brazilian panorama of the obese and overweight population has grown significantly in the last 30 years and tends to grow even more, in both sexes. This fact is due in large part to the sedentary lifestyle and the change in the dietary pattern, facilitated by easy access to industrialized foods, usually rich in hydrogenated fat and simple carbohydrates, with high palatability and low satiety power. Thus, prevention and early diagnosis of obesity or overweight are important aspects in health promotion and prevention. Due to this, among the medical students of a medical university, the idea was to hold a round table that contemplated several areas of Health and was open to the population. The event was held open to all academics and the local community. Part of the audience responded to a questionnaire about the importance of the event, knowledge gained from the talk and whether it will lead to some change in the participant's lifestyle. As a result of the 98 interviewees, $88,7 \%$ were female, the mean age was 22.82 years. About $95 \%$ were able to report their weight and height and $94 \%$ were able to calculate their BMI, but only $8.16 \%$ considered themselves obese. Besides that, $13,2 \%$ were discriminated by their weight; $32.6 \%$ reported eating fatty foods and $8,16 \%$ drank soda frequently. Finally, $98 \%$ believe that the professional speakers are fundamental for the process of weight loss; $93,8 \%$ said that they would change their lifestyle and $100 \%$ believe in the importance of the topic.

102. HEALTH PREVENTION FROM THEORY TO PRACTICE: OBSERVATIONAL ANALYSIS IN AN AMAZONIAN RIVERSIDE COMMUNITY.

Túlio Aguiar Farias, Letícia Machado Baptistella, Marcelo Motta Dutra.

Aiming at health prevention through a theoretical technical foundation, the present work is the result of an intervening experience in a community located in the low part of Madeira river through a partnership of an Academic Center of an institution of higher education with NAPRA (Núcleo de Apoio à População Ribeirinha) the Nucleus of Support to the riverside Population. This experience allowed an observational analysis of a mission to support the community to consolidate the training of students and professionals for voluntary action in the Amazon context. A livelihood analysis was carried out, noting the precariousness of the socioeconomic conjunctures, leading to a worsening of the living conditions of the population. It was also possible to identify the need for empowerment of the population on the importance of improving their health determinants through the triad: medical, government and population.
103. UNIVERSITY EXTENSION PROMOTED BY THE LEAGUE OF THE KIDNEY AND ARTERIAL HYPERTENSION OF BOTUCATU IN THE PROJECTS OF LITERACY OF YOUNG PEOPLE AND ADULTS AND "ACONCHEGO".

Beatriz Preto Almirall Seliger, Carlos Henrique Bianchi Florindo, Matheus Antônio Filiol Belin, Larissa Ramos Araújo, Luis Cuadrado Martin.

INTRODUCTION: Chronic Kidney Disease (CKD) is characterized by permanent loss of renal function, being related to underlying diseases, such as Systemic Arterial Hypertension (SAH) and Type 2 Diabetes Mellitus (DM2). The extension activity carried out by the Kidney League and Arterial Hypertension of the Faculty of Medicine of Botucatu, sought to raise the awareness of young and adult literacy (AJA) students about renal involvement and underlying diseases that lead to CKD. GOALS: through a continuing project of Health Education, to empower AJA students about CKD and their underlying diseases so that they could, through information and daily actions, prevent the disease. METHODOLOGY: Four activities were elaborated, with explanatory theoretical explanation and accessible language. In order to raise awareness about hypertension and DM2, diseaserelated images were taken and the relationship between the figures and diseases and CKD was explained later. In the third activity, a banner about healthy eating and physical activity was taken, having been explained in detail to the students. In the fourth activity, a class was elaborated to inform about the function of the kidneys and about what the CKD is. RESULTS AND DISCUSSION: We found that there is a great disinformation regarding CKD and its relationship with the underlying diseases, SAH and DM2. In addition, the activity was facilitated by the fact that the students already had, due to their experiences, contact with the diseases, which made possible an exchange of experiences in a dialogical way. CONCLUSION: It was possible to infer how unsatisfactory is the knowledge of the population about CKD and its underlying diseases. Therefore, the activity became important, since it took information to these individuals and empowered them on the problematic. Thus, they can spread the knowledge built in their social circles.

104. PHOMA INFECTION IN PEDIATRIC PATIENT.

Thaís Costa Reis Rodrigues, André Giglio Bueno, Giovanna Barille, Victor Carcia Nuñez.

Black fungus infections, also called pheohypomycosis, are in most of the cases diagnostic and therapeutic challenges despite their low incidence. Fungi of the genus Phoma sp. are possibly the most likely etiological agents. Like many other genera of black fungi, they are microorganisms found in soil, air, and aquatic environments. Cenerally, they are pathogenic to plants but, in some cases, to animals too. The major fungal infections of the genus Phoma described are subcutaneous mycoses, eye infections and rarely invasive infections. In this report, we describe an phoma infection in a pediatric patient, showing the factores related to infection and the case evolution.

\section{MARCHIAFAVA-BIGNAMI DISEASE: A RARE ENTITY TO BE REMEMBERED} IN CASES OF PELLAGRA ASSOCIATED WITH CHRONIC ALCOHOLISM.

Beatriz Rossi Barros.

Marchiafava-Bignami disease is a rare condition, associated with alcoholism, characterized by demyelination and necrosis of the corpus callosum. It has a predominantly radiological diagnosis, since the clinical characteristics are variable and not specific. We describe the case of a chronic alcoholic patient who presented Pellagra with typical cutaneous lesions: erythema on the surfaces exposed to the sun, on the extremities, on the face (erythema on "butterfly wing") and around the neck, as well as neuropsychiatric symptoms such as insomnia, depression and emotional instability. The patient was treated by clinical diagnosis of Pelagra with adequate vitamin replacement, regression of cutaneous lesions and improvement of neuropsychiatric symptoms. A few months later, the patient attended for clinical evaluation, without alcohol intake, with good nutritional support, but with symptoms of dementia. The Brain Magnetic Resonance Imaging (MRI) revealed radiological changes compatible with the Marchiafava-Bignami Disease. There are rare descriptions of the association of this disease and Pellagra in the literature, but since they share the alcoholic etiology, this possibility should not be neglected, especially in cases with acute symptoms that can lead to death. Dermatology plays a vital role in the identification of these cases, at an earlier stage, when associated with Pellagra. 
106. NEVO DE OTA: A DERMATOLOGICAL LESION WITH POSSIBLE CATASTROPHIC IMPACT ON THE PATIENT'S LIFE. Beatriz Rossi Barros.

Nevo de Ota (NO) affects about $0.4 \%$ to $0.8 \%$ of Asians. However, it is rarely present in Caucasian populations. Pathologically, it is a melanocytic dermal hamartoma that presents unilateral or bilateral discoloration, brown or blue, in the facial skin innervated by the trigeminal nerve. Since the development of laser technology, several have been introduced in the treatment of benign pigmentary lesions. Notably, Q-switched (QS) laser systems have gained popularity in the treatment of NO because they produce fewer scars and better results. It is difficult for dermatologists to choose the best solution in practice and high costs, and frustration with insufficient results leads to discontinuation of followup and severe psychiatric disorders due to social stigma. Research shows that physical appearance is one of the main drivers of personal well-being. In quality of life (OOL) instruments in dermatology, patients with face lesions demonstrated similar levels of social, psychological and emotional stress as those reported in patients with complicated asthma, epilepsy and diabetes. We present a case of a Caucasian teenager, with frustration after several treatments followed, including numerous laser therapy sessions, which show social isolation and suicidal ideation. Treatment should from the outset involve Dermatology, Psychiatry and Psychology.

\section{KLIPPEL-TRENAUNAY SYNDROME: A RARE DISEASE REVISITED BY} DERMATOLOGY.

Beatriz Rossi Barros.

Klippel-Trenaunay syndrome (SKT) was described in 1900 as a triad consisting of cutaneous capillary hemangiomas, bone and soft tissue hypertrophy, and venous dilatation. Port wine stain or flat hemangioma is a vascular malformation present at birth with a low probability of regression. SKT should be suspected in all newborns with capillary malformations involving the extremities of the body. There is no curative treatment, and the therapeutic objectives are to improve the patient's symptoms and stigmata. For port wine stains the treatment of choice is pulsed laser being performed early, since children require fewer sessions and have better results. We describe a case of an adolescent who was born with SKT and attended a dermatology service early with laser therapy treatment, but unfortunately without follow-up with an orthopedist and vascular surgeon. Sixteen years ago, she performed ten laser sessions with improvement of the affected limbs. Resolution of these port wine stains, defined as persistent bleaching of the lesion occurs in only $10 \%$ to $20 \%$ of cases. The patient benefited in thesis from a more efficient result by being treated early, opening the discussion that Klipplel Trenaunay Syndrome should be approached by a dermatologist soon after birth.

\section{NEONATAL SUPRAVENTRICULAR TACHYCARDIA: TWO CASE REPORTS} AND LITERATURE REVIEW.

Yasmim Nadime José Frigo, Luiza Ravanini da Cunha Claro, Jacqueline Scholz Berça, Cristina Sylos.

INTRODUCTION: Supraventricular tachycardia occurs in an incidence of 16 to 100 thousand newborns and there is a good prognosis if the diagnosis is made early and the oral treatment is adequate. The clinical status is nonspecific and varies among age groups, with signs and symptoms of irritability to palpitation. Early diagnosis and appropriate treatment are essential because of the risk of possible progression to heart failure after a variable period. METHODOLOGY: Retrospective analysis of the medical record of 2 patients with neonatal supraventricular tachycardia of our pediatric cardiology department. RESULTS: The supraventricular tachycardia is the arrhythmia in common to these two patients of the neonatal age group shown in this case report. The first patient had a better and faster response to the proposed clinical treatment, compared to the second, showing that the early diagnosis is associated with a better prognosis. CONCLUSION: 1) Sepsis is a differential diagnosis of supraventricular tachycardia in neonates. 2) The vagal maneuver can be used to reverse the clinical condition, and if ineffective, in stable patients, Adenosine can be used successfully. 3) The prognosis is good if the diagnosis is made early. 4) If supraventricular tachycardia is not treated or diagnosed, it may evolve to a tachycardiomyopathy and cardiogenic shock.
109. PHYSIOTHERAPY IN THE POSTOPERATIVE PERIOD OF THE TEMPOROMANDIBULAR JOINT: CASE REPORT.

Michelle de Figueiredo Luizão, Luiz Carlos Boaventura.

INTRODUCTION: The temporomandibular joint (TJM) is a synovial joint that allows movements of the mandible around the temporal bone. It is interconnected to the mandible containing simultaneous movements, being considered a single articulation. Changes in TMJ favor temporomandibular disorders (TMDs) with great influence in population. Many reports are of female people, aged between 20 and 40 years; women are more affected due to hormonal factors and to the fact that they usually seek more medical treatments than men (PEREIRA et al., 2005). OBJECTIVE: To report the importance of the physiotherapeutic approach in TMJ postoperative care together with a multidisciplinary team. METHOD: Analysis of the documentation of the bucomaxillofacial treatment for case report. The patient underwent orthognathic surgery and TMJ surgery due to complaints of constant headache, limitation of mouth opening and acute pain in the bilateral pre-auricular region, due to joint damage with anterolatera displacement of the right articular disc, without recapture of the lateral component to the mouth opening. Based on the case, articles describing the results of the physiotherapeutic interventions for patients with TMD were used. RESULTS: Supporting the jaw on the hand, bruxism, gum chewing biting the lips, onicophagy and biting objects are habits described as aggravating and reducers of the coordination of the affected muscles, stating the click as the most common symptom due to improper cartilage positioning accompanied or not pain. Due to the high incidence of signs and symptoms related to TMD, there is a need for an interdisciplinary follow-up to minimize or eliminate these symptoms, facilitating the physiotherapeutic treatment and improving the quality of life of the patient. CONCLUSION: There is a need for multidisciplinary work in order to improve pain and function, promoting the improvement of signs and symptoms.

\section{TOXIC MEGACOLON SECONDARY TO PSEUDOMEMBRANOUS COLITIS: A} CASE REPORT.

Izabela Barbieri De Freitas, Isaac Ferrari Del Favero, Luís Henrique Simões Covello, Luciana Coelho Sanches, Cristina Amendola Prata.

Clostridium difficile is an important community pathogen and the most frequent cause of nosocomial diarrhea associated with antibiotics. The incidence of $C$. difficile infection (CDI) has subsequently increased morbidity, mortality, and health care costs worldwide. The major risk factor for CDI development is exposure to broad spectrum antibiotics. The incidence of fulminant $\mathrm{CDI}$ is up to $3 \%$ of total CDI and has as main complications spontaneous colonic perforation and toxic megacolon. The present study aims to report the case of a 67-year-old patient with fulminant pseudomembranous colitis evolving with toxic megacolon, a rare case of great medical relevance, and that even after performing the rapid diagnosis and institution of appropriate treatment, the patient evolved with worsening of the clinical picture and death. In the case reported, the patient in question had risk factors that contributed to the patient's tragic condition, such as age greater than 65 years; immunosuppression due to chemotherapy and radiotherapy; and use of a broad spectrum antibiotic (cephalosporin). Therefore, C. difficile fulminant colitis is a cause of undervalued death secondary to its non-specific clinical syndrome and lack of general knowledge of the spectrum of clinical disease. So, the rapid diagnosis and the treatment are essential for a positive outcome, as well as early surgical intervention in order to avoid the rate of complications.

\section{CASE REPORT: SERVICE DOG AS PROTECTIVE FACTOR AGAINST SUICIDAL} IDEATION IN A PATIENT WITH PROBABLE DIAGNOSIS OF BIPOLAR AFFECTIVE DISORDER.

Emanuela Yumi Fugisawa de Mello, Fernanda Folla Pompeu Marques, Fernanda Milani, Tiago Genzini de Miranda, Márcia Braga Cliquet.

INTRODUCTION: Assisted Animal Intervention (AAI) consists of any intervention that intentionally includes animals in the therapeutic plan to improve the environment in which the patient lives or the pathological course itself. There are studies that demonstrate efficacy in several psychiatric pathologies; however, there are no reports of this application for patients diagnosed with Bipolar Affective Disorder (BAD). CASE REPORT: A 45- year-old male, in the process of divorce, from Votorantim, sought CAPS care with a complaint of sadness and lack of reasons to live, reporting a worsening of the condition for 5 years. He comes with the dog, reports two 
previous suicide attempts prior to the animal's possession. Patient demonstrates active attitude during the consultation with voluntary discourse, introspective body posture and depressive gaze, has self-care compromised. Demonstrates extreme bonding and emotional dependence with the animal. It reports interest and daily social activities dependent and related to the care of the animal. DISCUSSION: Suicide is an avoidable death cause where risk factors and protective factors are involved. A study conducted in Taiwan with 1542 patients with bipolar depression and 17480 patients with other types of depression concluded that bipolar depression is associated with a double chance of suicide and practically double chance of accidental death when compared to patients with another type of depression. The treatment aims at the prevention of attempts and uses pharmacotherapy and psychotherapy. CONCLUSION: Although there are no specific studies to verify the effectiveness of AAI in individuals diagnosed with $B A D$, it is known that the main effect of the emotional support animal is to alleviate the symptoms present in the various psychiatric disorders and it is plausible to associate AAl with pharmacological and psychotherapeutic standard treatment, increasing the chances of successful treatment and decreasing the risk of suicide attempts.

\section{PRODUCER ECTOPIC TUMOR ACTH: CASE REPORT.}

Julia Brasileiro de Faria Cavalcante, Pedro Nogarotto Cembraneli, Renata Brasileiro de Faria Cavalcante, José Edison da Silva Cavalcante, Fabio de Azevedo Caparroz.

INTRODUCTION: Intrinsic pituitary adenomas secreting ACTH are the most common cause of Cushing's Syndrome. Rarely, the pituitary adenoma may be ectopic, that is, located outside the gland. Supra-dependent (ectopic) ACTH pituitary tumors are extremely rare tumors, with few cases described in the literature to date. Most ACTH dependent cases are caused by pituitary adenomas (90\%), while ectopic adenomas occur in approximately $10 \%$ of cases. Accurately locating the source of adrenocorticotrophin secretin lesion is crucial to solving the condition by removing the ACTH-producing primary tumor. This type of tumor has a higher prevalence in the female sex $(62 \%)$, with an average age of 50 years. Therefore, it was decided to report a case of a patient who was diagnosed with a suprasellar tumor attached to the pituitary stalk, and a craniotomy was necessary. CASE REPORT: A 19-year-old male patient with a $29 \mathrm{~kg}$ gain in two years, associated with acne and striae without definite cause. The hypothesis of Cushing's Syndrome was made. He then asked for laboratory tests for diagnostic confirmation. In addition, magnetic resonance imaging (MRI) of the turkish saddle was visualized and a suprasellar tumor $0.5 \mathrm{~cm}$ in diameter adhered to the pituitary stalk. The tumor was then excised by craniotomy. DISCUSSION: There are two types of endogenous causes of hypercortisolism, independent and dependent ACTH. The dependent form may originate from a pituitary tumor producing ACTH $(80 \%)$ or ectopic adenoma producing ACTH $(20 \%)$. Despite the fact that, currently, most cases are approached endoscopically endonasal, surgical treatment via craniotomy can be performed in selected cases. CONCLUSION: ACTHdependent ectopic tumors are rare. One should be aware of the possibility of ACTH-dependent pituitary tumors as an etiologic factor in Cushing's Syndrome.

\section{FOURNIER'S SYNDROME: CASE REPORT.}

Julia Brasileiro de Faria Cavalcante, Pedro Nogarotto Cembraneli, Francisco Estefani Segato, João Vitor Soares Vicentini, Rafael Luís Luporini.

INTRODUCTION: Fournier's syndrome is a polymicrobial infection that mainly affects a perianal, perineal and genital region. It manifests insidiously, painfully, erythema, fever, crackling, shock and altered level of consciousness. Some risk factors, such as diabetes mellitus, hypertension, obesity, smoking, alcoholism and immunosuppressive diseases may be associated. The diagnosis is clinical and should be performed early Treatment is based on three pillars: initial patient stabilization, broadspectrum antibiotic therapy, and extensive surgical debridement of the entire necrotic area. Therefore, it was decided to report a case of diagnosis with Fournier's Syndrome, requiring treatment with broad spectrum antibiotic therapy, surgical debridement, and hyperbaric sessions. CASE REPORT: male patient, 64 years old, with complaint of perianal and scrotal abscess for 15 days, associated with purulent discharge, asthenia and unmeasured fever. Fournier's syndrome was hypothesized. Initiated broad spectrum antibiotic therapy and performed debridement of necrotizing perineal fasciitis, protective transversostomy in addition to the onset of hyperbaric oxygen therapy, adjuvant to treatment. DISCUSSION Most often, this syndrome is caused by an anorectal infection of genital origin, with perirectal abscess being the most frequent, especially when there is a delay in diagnosis. The diagnosis is clinical and should be done early. The treatment must be done immediately, being aggressive and with intensive support. This treatment aims at the extensive removal of devitalized tissues, in addition to interrupting the progression of the infectious process. CONCLUSION: With the present study, we aimed to alert emergency doctors regarding the clinical picture of this disease, as well as its complications and the importance of performing the diagnosis early. In addition, this disease is a surgical emergency, requiring immediate intervention.

\section{HIRSCHSPRUNG'S DISEASE: CASE REPORT}

Pedro Nogarotto Cembraneli, Julia Brasileiro de Faria Cavalcante, Francisco Estefani Segato, João Vitor Soares Vicentini, Rafael Luís Luporini.

INTRODUCTION: Hirschsprung's disease (DH) is a megacolon due to obstruction, most often of the rectum and sigmoid colon. Currently, it consists of the congenital absence of glandular cells of the myenteric and submucosal plexuses of the digestive tract, leading to a functional intestinal obstruction. To elucidate the diagnosis, it is necessary to perform complementary exams, but the gold standard is the rectal biopsy. The treatment is always surgical, aiming at the removal of the aganglionic part and restoration of the continuity of the intestine. With this, it was chosen to report a case of a 1-year and 10-month-old patient with chronic constipation since birth, who was diagnosed with HD, requiring surgical intervention. CASE REPORT: A male patient, 29 days old, presenting with small bowel movements from birth, with yellowish, pasty stools with a characteristic odor, intercalating with periods of constipation. Mother reports that the RN remained for up to a week without evacuating. With the DH hypothesis, an abdominal x-ray, an opaque enema, and finally a rectal biopsy confirmed the hypothesis. We opted for surgical treatment where initially a colostomy was performed in the loop and in another procedure, a rectosigmoidectomy with colon retraction. DISCUSSION: DH is a congenital anomaly that affects 1 in 5,000 live births. It is characterized by the absence of ganglion cells, and 80 to $90 \%$ of the cases are diagnosed in the neonatal period. The delay between the onset of the clinical manifestations and the investigation of the disease increases the risk of complications, being more frequent the enterocolitis, besides increasing the morbimortality of the congenital megacolon. CONCLUSION: With the present case report, we aim to emphasize the need to alert the pediatrician for a better understanding of the clinical picture of $\mathrm{DH}$, as well as its complications and the importance of performing the diagnosis early.

\section{ECTOPIC TWIN TUBAL PREGNANCY: CASE REPORT.}

Arthur Vicensoto Fukuhara, Ademir Roberto Sala, Celina Gai Höehr, Flávia Vicentin Silva.

The ectopic pregnancy refers to a complication during pregnancy in which the implantation of the blastocyst occurs in an ectopic site. It is a common cause of morbidity and mortality for women at reproductive age. In cases of ectopic pregnancy there are cases of twin ectopic pregnancy, usually intercurrent in the uterine tube, being extremely rare ( 1 in 20,000 cases) This study presents a case of a child with twin tubing pregnancy, with presence of fetal heart beat. The patient underwent exploratory laparotomy and salpingectomy. The surgery was performed without intercurrences and in the postoperative period the patient was medicated with analgesics.

\section{COEXISTENCE OF ADULT T-CELL LEUKEMIA / T-CELL LYMPHOMA AND TROPICAL SPASTIC PARAPARESIS ASSOCIATED WITH HTLV-1 VIRUS: CASE REPORT.}

Arthur Vicensoto Fukuhara, Ana Carolina Lara Ferrão Cunha, Cibele Repele Duch.

The Human T-cell lymphotropic virus (HTLV) is a RNA virus with tropism by T lymphocytes discovered in the 80's. Nowadays, around 20 million people worldwide are affected by the virus and Brazil has the highest absolute number of cases ( 2.5 million). However, only $5 \%$ of infected individuals will develop any disease related to the virus, while the vast majority will continue asymptomatic, but able to transmit it by sexual, parenteral and vertical ways. The development ofdiseases related to HTLV as Adult T-cell 
Leukemia / Lymphoma (ATL), myelopathy, uveitis, rheumatoid arthritis, infectious dermatitis, among others, will depend on the interaction between virus proviral load, host and environment. This report will present the case of a patient whichevolved both complications: a 49 years old woman, single, white, domestic worker and diagnosed with HTLV-1 and Tropical Spastic Paraparesis (TSP) six years before internment for medical examination to investigate hers gait and ascending paresis of the lower limbswhich she presented since youth. In April 2017, the patient was admitted to Santa Casa de Araraquara with leukocytes $102.400 / \mathrm{mm} 3$ and $80 \%$ of lymphocytes, fever, asthenia, abdominal pain and photophobia of one month of evolution. The ATL's suspect was confirmed by leukocytes' immunophenotyping with flow cytometry. Then, the acute lymphoblastic leukemia (ALL)'s treatment began with chemotherapy combined to anti neoplastic's drugs, according to HyperCVAD's protocols.The patient developed severe pulmonary infection probably caused by a fungal infection, sepsis orrefractory septic shock, and died after 40 days of hospitalization. As known, the leukemic stage of HTLV's infection is aggressive and the treatment has intense regimens, while improving survival, brings increased treatment-related mortality. Considering that the prevalence of HTLV is high in Brazil, surveillance of the transmission and prevention routes should be constant, because there are serious complications with high morbimortality.

\section{LAPAROSCOPIC TREATMENT OF GIANT CIRCUMFERENTIAL LEIOMYOMA} OF THE DISTAL ESOPHAGUS: CASE REPORT.

Luana Cabrino Aranha, André Brandalise, Nelson Ary Brandalise, Letícia Gomez Gallego, Nilton Cesar Aranha.

INTRODUCTION: Leiomyoma is a rare esophageal tumor, with an incidence of $0.4-1 \%$ of esophageal tumors, but it is the most common benign tumor of this site, corresponding to $67-80 \%$ of the cases. About half of the tumors are smaller than $5 \mathrm{~cm}$ and only $5 \%$ of these tumors grow to the point of being called giant, with diameters greater than $10 \mathrm{~cm}$. Most often asymptomatic, the onset of dysphagia, odynophagia, retrosternal pain and bleeding usually appear in tumors equal to or greater than $5 \mathrm{~cm}$. Tomography determines the size and can be used for periodic monitoring of incidentalomas, since this is a benign tumor of slow growth. CASE REPORT: VCA, female, 38 years old, reported having had a CT scan at another service 5 days prior due to a complaint of pain in the posterior dorsum to the right. She also presented chest pain and a sense of retrosternal oppression during four months. The $\mathrm{CT}$ result showed lobulated parietal thickening in the distal esophagus, nonspecific, of origin in the submucosa and suggestive of leiomyoma. At the examination, there were $3 \mathrm{~cm}$ of parietal thickness, 6.9 $\mathrm{cm}$ in the largest axial axis and $5.3 \mathrm{~cm}$ in extension. Patient presents a history of ankylosing spondylitis since the age of 19. Underwent an endoscopy four months ago, at the request of a rheumatologist, for a complaint of heartburn, mild dysphagia, and a sensation of stopping the medication in the esophagus (sulfasalazine). It was determined a widening hiatus gap of two centimeters and mild gastritis. The hypothesis of removal of the tumor lesion by laparoscopic transhiatal approach was considered given its location and, if necessary, partial esophagogastrectomy with transmediastinal gastric tube reconstruction.Patient was submitted to excision of the complete tumor lesion without mucosal lesion with preservation of vagal nerves, by videolaparoscopic method. Reconstruction of the external muscular wall was performed by continuous suture with the presence of orogastric Fouchet probe of $32 \mathrm{fr}$ to avoid constriction of esophageal light. Also, complemented by the accomplishment of a hiatoplasty and partial esophagogastrofundoplicature with the extension of $7 \mathrm{~cm}$ covering the whole esophageal area from where the lesion was resected. The anatomic examination confirmed the esophagus leiomyoma with $10 \times 5.0 \times 2.0 \mathrm{~cm}$. Evolving clinically in the long term without the appearance of heartburn or dysphagia. After 6 months of surgery, patient was submitted to control by contrast examination of the esophagus, without esophageal constriction and absence of reflux. At the end of 1 year, the endoscopy examination showed a good anti-reflux valve and normal tomography. DISCUSSION: The main indication for surgical removal of leiomyoma of the esophagus is the appearance of symptoms. The classic treatment of giant leiomyoma, greater than $10 \mathrm{~cm}$, includes thoracotomy with esophagectomy and esophagogastrostomy. Thoracotomy significantly increases morbidity, with greater respiratory complications, longer time to reintroduce the diet, greater surgical trauma, and complications related to anastomosis, which increase hospitalization time and worsen prognosis. With the advances of minimally invasive surgery, there are already some cases of thoracoscopic treatment described. Considering that in $80 \%$ of the cases the leiomyoma of the esophagus is found in the middle and lower thirds of the esophagus, the videolaparoscopic approach by transhiatal route becomes possible. The reconstruction of the external muscle is important to prevent a possible prolapse of the mucosa. In the case described, the transhiatal route still allowed the correction of the gap widening of the patient in question. The realization of fundoplication, besides avoiding gastroesophageal reflux, allows the suture area of the muscular wall of the esophagus to be covered, thus increasing safety in the surgical treatment. CONCLUSION: In those services with adequate experience in performing surgical treatment of gastric esophagus transitional diseases by videolaparoscopic method, surgical treatment of tumor lesions of the distal esophagus can be performed with adequate safety. The transhiatal approach allows for excision of giant and circumferential leiomyomas of this region. The method provides the patient with greater postoperative comfort and prompt clinical recovery.

\section{PANFACIAL TRAUMA WITH SURGICAL CORRECTION: CASE REPORT.}

Flávio de Fava Sanches, Natália Butturi Varone, José Mauro Rodrigues.

Panfacial fractures are the most complex of the face because they concomitantly affect the lower, middle and upper thirds. They originate from high-energy trauma and involve structures such as the frontal bone, zygomatic complex, naso-orbito-ethmoidal complex, maxilla and mandible. often these lesions are related to soft tissues and the loss of structures can lead to severe facial deformities and functional impairment. According to studies, the male gender has been more prevalent and the principal causes of this type of fracture are automobile accidents, trampling, interpersonal traumas, among others. Patients with this type of injury usually have involvement in other vital structures. This implies a delay in the surgical correction of facial fractures, being performed as soon as the patient is clinically stabilized. The objective of the study is to discuss how the delay in the surgical management of patients with panfacial fractures can compromise the restoration of facial function. The authors of this article report the case of a male patient, 21 years old, a victim of a motorcycle accident. After the clinical, physical and radiological examination in the hospital emergency, fractures were found in a naso-orbito-ethmoidal frontofacial complex, bilateral orbital zygomatic complex, zygomatic arch D and Le fort. II and III. He remained under observation for 12 days in the ICU, and after that, surgery was performed to reduce fractures. It is possible to conclude that correct management in primary attendance, the accurate diagnosis, the planning to reduction and osteosynthesis of the fractured segments, as well as post-operative clinical and radiographic follow-up are essentials requirements for successful treatment of panfacial fractures. Finally, the time of initial treatment and the quality of the first intervention proved to be two of the most important factors to optimize the restoration of facial function.

\section{RAPIDLY PROGRESSIVE COCAINE-INDUCED GLOMERULONEPHRITIS.}

Yara Franceschi Saba, Cabriele Escocia Marinho, Alexandre de Toledo Arrebola.

OBJECTIVE: To report the case of a patient who developed rapidly progressive glomerulonephritis whose early diagnosis and therapeutics are fundamental for a favorable outcome of the disease. DISCUSSION: Male patient, 33 years old, cocaine user, presenting asthenia, altered taste, nausea, medium-duty dyspnea, lower limb edema, arterial hypertension, with progressive reduction of urine output until anuria. Intake exams: creatinine $30.5 \mathrm{mg} / \mathrm{dL}$ and urea $272 \mathrm{mg} / \mathrm{dL}$. Table interpreted as rapidly progressive glomerulonephritis possibly due to abusive use of impure cocaine containing levamisole. Renal biopsy shows membranous glomerulonephritis with crescents. Treatment with partial recovery of renal function was started. Dual glomerulopathy in the scenario of chronic cocaine abuse is rare, with few cases reported in the literature. Recently, in the United States, the anti-drug agency reported that $69 \%$ of cocaine seized was contaminated with levamisole. This substance has been recognized as an important inducer of glomerular damage, the precise mechanism remains unclear, it is known to date that it causes agranulocytosis which may be accompanied by increased release of extracellular neutrophil traps. Reports suggest that it may bind to its own antigens leading to autoimmunity. Glomerulopathy can also be caused by the deposition of exogenous antigen, levamisole. The lack of documentation of the contaminant in patient samples is clearly a limitation to the current case. CONCLUSION: Rapidly progressive glomerulonephritis is an uncommon, prognostic entity that should be suspected in all cases where there is a rapid loss of renal function. Treatment should be instituted as early as possible. 
120. CAN FABRY'S DISEASE BE ASSOCIATED WITH DOMINANT AUTOSOMAL DOMINANT POLYCYSTIC KIDNEY DISEASE IN ADULTS? CASE REPORT. Carolina Kath Lucca, Andréia Cristina Tassi, Andrei de Camargo Carotti, José Ricardo Fraçon Viana Alves, Leandro Junior Lucca.

INTRODUCTION: Fabry disease is an innate error in the metabolism of globotriaosylceramide (lyso GB-3) bound to the $X$ chromosome and secondary to a deficient activity of the $\alpha$-galactosidase A lysosomal enzyme, resulting in excessive intracellular storage of lyso CB-3. The affected organs include the vascular endothelium, the heart, the brain and the kidneys, leading to organ failure and premature death. There is a higher frequency of parapyelic cysts in patients with Fabry disease in the literature, however there is no association between Fabry disease and autosomal dominan polycystic kidney disease in adults. OBJECTIVE: The objective of this study is to report the case of a patient with FD and autosomal dominant polycystic renal disease of the adult, as well as of the family, in a follow - up at the Instituto Bebedouro de Nefrologia - IBENE. Method: Information was obtained by reviewing the medical record, interviewing the patient and family, and reviewing the literature. FINAL CONSIDERATIONS: the case reported and publications raised bring to light the discussion of the diagnosis of therapeutics of a complex situation, which is Fabry's Disease.

121. ENDOCARDITIS, VASCULITIS AND SPONDYLODISCITIS SUGGESTING TUBERCULOSIS WITHOUT PULMONARY INVOLVEMENT: CASE REPORT. Thaís Cristina Faria Pacheco, Arthur Manzani Fernandes, Ermeson Fellipe Rodrigues Ramos, Otacílio de Camargo Júnior.

Tuberculosis is a chronic infection which incidence is high in Brazil. Although it frequently involves the lungs, extrapulmonary presentations may mimic vascular diseases and several others. Objective: report the case of a patient who presented endocarditis, vasculitis and dyscite, suggesting tuberculosis without pulmonary involvement. Method: the information was obtained by reviewing the patient's medical record, interviewing the patient during admission, photographic record of the diagnostic methods to which the patient was submitted, and review of the literature. Report: A 34-year-old female patient was admitted to a tertiary hospital in order to investigate Takayasu's arteritis suspect, presenting, among other symptoms, lower limb pain and cyanosis in lower left limb toe joints. During hospitalization, she started to present manifestations of endocarditis, vasculitis and spondylodiscitis, all of which were compatible with tuberculosis, although rare in patients without pulmonary disease and immunocompetent. Conclusions: Tuberculosis is an important differential diagnosis of Takayasu's arteritis, although it is often difficult to diagnose. We must pay attention to the extrapulmonary manifestations of tuberculosis, which may mimic several other diseases.

122. SEVERE PROTEIN MALNUTRITION ASSOCIATED WITH AN INFECTIOUS CONDITION IN THE LATE POSTOPERATIVE PERIOD OF BARIATRIC SURGERY: CASE REPORT.

Alice Avesani Cavotto Furlan, Marcia Varella Morandi Junqueira Franco, Selma Freire de Carvalho da Cunha.

The purpose of this report is to describe the evolution of a patient submitted to two bariatric surgeries who had low adherence to the diet and the vitamin and mineral supplementation. She manifested severe protein malnutrition, vitamin and minerals deficiencies in the late postoperative period. A hospital admission, there was severe hypoalbuminemia (albumin: $1.5 \mathrm{~g} / \mathrm{dL}$ ) edema, ascites, as well as the growth of Streptococcus sp subcutaneous abscess in the foot. The patient was given antibiotic injections, vitamins $A$ $D, B 1, B 12$, thiamine, calcium and parenteral nutrition. During 28 days a the hospital, there was a decrease in body weight $(18.4 \mathrm{~kg})$ attributed to a drastic reduction of total body water $(25.3 \mathrm{~L})$. Parenteral nutrition was suspended after 25 days, and oral diet was kept fractional and fiberrestricted. In outpatient care, there was a gradual improvement in laboratory data, which were close to the reference values. Such outcome shows the need for specialized care in preventing and treating nutritional complications after bariatric surgeries as well as atypical clinical manifestations of infection in previously undernourished patients.

\section{TAKOTSUBO CARDIOMYOPATHY: CASE REPORT.}

Eloa Scalfi Caproni, Felipe Menezes Santos, Karine Evangelista Pascoaleto, Mayra Christina Nagata, Jose Guilherme Rodrigues de Paula.
Takotsubo cardiomyopathy is about a pathology that looks like an acute coronary syndrome (ACS). It is more common in women, usually menopausal, exposed to physical or emotional stress. It presents undefined pathophysiology and the diagnosis is made through coronary angiography demonstrating coronary arteries free of obstructive lesions or significant non-obstructive Coronary Artery Disease (CAD) with impairment of ventricular segmental function through complementary examination (ventriculography and/or echocardiogram evidencing hypokinesia apical and/or extensive left mesoventricular with the contractility of the preserved or hyperkinetic basal portion of the left ventricle (LV)).

\section{CASE REPORT: CYSTIC FIBROSIS AND THE IMPORTANCE OF EARLY} DIAGNOSIS.

Letícia Nascimento Machado, Caroline Cestari, Mariana Morais Olimpio, Carolina Magalhães Britto Rodrigues, Mariana Conçalves Comes, Ana Beatriz Alves.

Cystic fibrosis is a genetic disease, predominant in Caucasians, with a multisystemic and common manifestation in the first years of life. It presents an estimated prevalence of 1: 3,500 to 1: 10,000 live births in Brazil. Its pathophysiology is explained by a dysfunction in the CFTR gene, promoting changes in exocrine glands at different levels. The reported case presents a patient diagnosed from the positivity of the neonatal screening, which allowed for a greater early knowledge about the disease by the family, better treatment and successful resolutions of respiratory disorders, infectious disorders and difficulty of weight gain throughout the follow-up. the improvement of neonatal screening and its expanded access to an early diagnosis, allowing better longevity, better quality of life and lesser future consequences. In addition, to ensure a good prognosis, treatment must be multiprofessional and continuous.

125. P.A.R.T.Y. (PREVENTION OF TRAUMA RISK RELATED TO ALCOHOL USE IN YOUTH) AS AN EXTENSION PROGRAM OF THE TRAUMA LEAGUE OF UNICAMP.

Roberta Rocha Gomes, Breno Kobayashi Hiratsuka, Diego Mazzoli Gutierrez, Ana Paula da Silva Rios, Thiago Rodrigues Araujo Calderan.

The program P.A.R.T.Y. (Prevention of Alcohol-Related Trauma Risk in Youth) was created in Toronto, Canada, motivated by high rates of alcohol-related teenage and teenage trauma. The initiative has been expanded to many other countries. In Brazil, it was initially implanted in 2008 , at the Medical School of Ribeirão Preto (FAMERP). In the Faculty of Medical Sciences of the State University of Campinas (FCM-UNICAMP) the program was implemented in 2010, and is already in its 132nd edition and counted with the participation of 7260 students from the public schools of Campinas, from 15 to 18 years of age. P.A.R.T.Y. aims to make young people aware of the responsibility and consequences of their choices when it comes to the direction and use of alcohol. The program is very effective because the majority of students leave the experience with a much more conscious and responsible view on trauma.

\section{USE OF LICIT AND ILLICIT DRUGS BY MEDICAL STUDENTS IN BRAZIL.} Arthur Petta.

INTRODUCTION: The use of drugs is present and widespread throughout the population and there has been a certain increase in habit in some groups with emphasis on university students and specially medicine students. To know the main individuals exposed to this factor of risk for various diseases is essential to design therapeutic approach strategies. OBJECTIVE: To analyze drug use among medical graduates of several universities of Brazil according to publications from 2000 to 2018. METHODOLOGY: This is a integrative review of a bibliographic study, with quantitative and qualitative analysis, based on articles published between 2000 and 2018, in scientific databases such as Bireme, Lilacs, Scielo, and MEDLINE, using the following descriptors: Inhalant Abuse, Consumption of Tobacco-Derived Products, Alcohol Drinking in College, Street Drugs, Students, Medical. RESULTS: Six articles were selected, most published in journals in the area of psychiatry or medical education, between 2000 and 2010. In 10 years, from 2000 to 2010, alcohol consumption remained practically constant, but oscillated during this period. In the case of tobacco and inhalants, however, there was a decrease in those 10 years, but there were also oscillations in the consumption of these drugs during this period. CONCLUSION: Knowing the particularities of 
Abstracts

XXVII Medical Academic Congress of Unicamp, CoMAU, 2018

drug use among university students is of paramount importance for the early detection of this consumption, the structuring of strategies / programs to minimize this consumption and support the students.

This work is licensed under a Creative Commons Attribution 4.0 International License

ISSN 2076-6327

This journal is published by the University Library System, University of Pittsburgh as part of the Digital Publishing Program and is co-sponsored by the University of Pittsburgh Press. 